\title{
Adaptive Forward-Inverse Modeling of Reservoir Fluids Away from Wellbores
}

\author{
Said K. Doss \\ Robert J. Gelinas \\ Robert G. Nelson* \\ John P. Ziagos
}

*PDE Solutions, Inc.

Phase I Final Report

February 1999

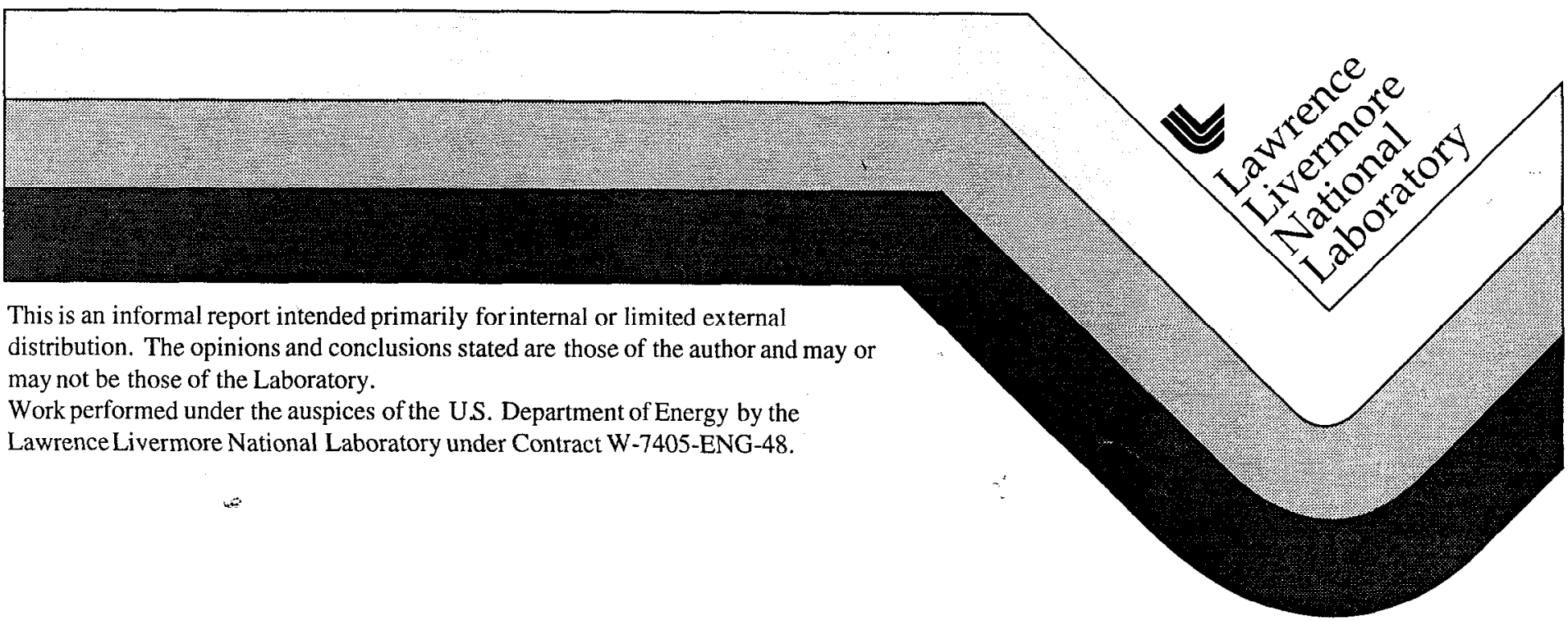


This document was prepared as an account of work sponsored by an agency of the United States Government. Neither the United States Government nor the University of California nor any of their employees, makes any warranty, express or Implled, or assumes any legal liability or responsibility for the accuracy, completeness, or usefulness of any information, apparatus, product, or process disclosed, or represents that its use would not infringe privately owned rights. Reference herein to any specific commercial products, process or service by trade name, trademark, manufacturer, or otherwise, does not necessarily constitute or imply its endorsement, recommendation, or favoring by the United States Government or the University of California. The views and opinions of authors expressed herein do not necessarily state or reflect those of the United States Government or the University of California, and shall not be used for advertising or product endorsement purposes.

This report has been reproduced directly from the best available copy.

Available to DOE and DOE contractors from the Office of Scientific and Technical Information P.O. Box 62, Oak Ridge, TN 37831

Prices available from (423) 576-8401

Available to the public from the National Technical Information Service

U.S. Department of Commerce 5285 Port Royal Rd. Springfield, VA 22161 


\section{Table of Contents}

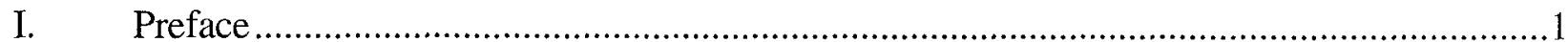

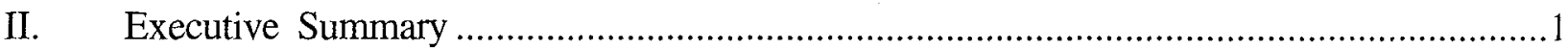

III. Statement of the Problem and Overview............................................................................

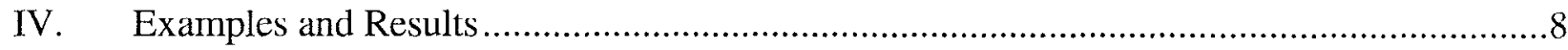

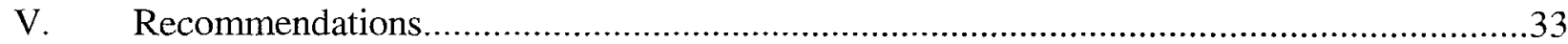

\section{List of Appendices}

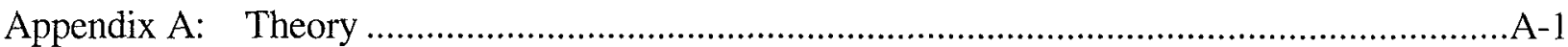

Appendix B: Analytic Description of the F-I Method.............................................................

Appendix C: Description of the FLEXPDE Toolkit..................................................................

Appendix D: Listing of the Problem Setup Commands .......................................................... D-1

Appendix E: Black Oil Model for Example Problems ...............................................................

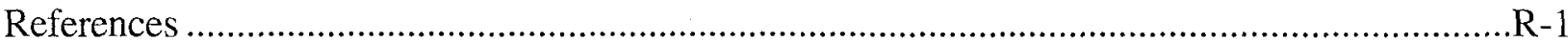

\section{List of Figures}

Figure 1. 2-D horizontal oil production water injection model .............................................10

Figure 2. Isocontours of water saturation after 100 days......................................................13

Figure 3. Corresponding unstructured adaptive grid distribution after 100 days ....................13

Figure 4. Isocontours of water saturation after 200 days......................................................

Figure 5. Water saturation at break-through after 340 days ................................................14

Figure 6. A blow-up of the water saturation front arrival at extraction well (see Figure 4)......15

Figure 7. Production curve (red) and normalized Production Rate curve (green) calculated at the extraction well from $\mathrm{t}=0$ to 1000 days..........................................................15

Figure 8. 2-D vertical oil production water -drive model .....................................................16

Figure 9. Isocontours of water saturation after 100 days with $\mathrm{R}=1 / 10 \ldots \ldots \ldots \ldots \ldots \ldots \ldots \ldots \ldots \ldots \ldots . .18$

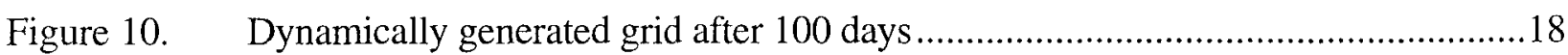

Figure 11. First time arrival of water break-through after 200 days......................................19

Figure 12. Blow-up of first time arrival of water break-through at extraction well....................19

Figure 13. Second water break-through at 220 days ............................................................20

Figure 14. Blow-up of second water break-through at extraction well.....................................20

Figure 15. Figure 15 - Isocontours of water saturation after 100 days for $R=0 \ldots \ldots \ldots \ldots \ldots \ldots . .21$ 


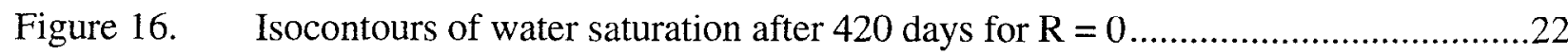

Figure 17. Recovery of K from densely sampled pressure data; 834 nodes; Stage 1 .............25

Figure 18. Recovery of $\mathrm{K}$ from densely sampled pressure data; 1,717 nodes; Stage 2...........25

Figure 19. Recovery of K from densely sampled pressure data; 2471 nodes; stage 3 .............26

Figure 20. Isocontours of densely sampled pressure data $(t=0+$ snapshot $)$.......................26

Figure 21. Calculated permeability image from approximately 300 pressure datum points, with

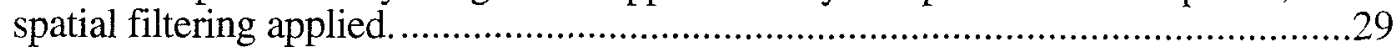

Figure 22. Calculated permeability image from approximately 75 pressure datum points, with

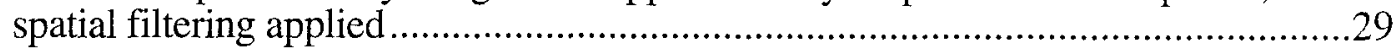

Figure 23. Calculated permeability image from approximately 20 pressure datum points, with spatial filtering applied ....................................................................... 30

Figure 24. Calculated permeability image from one pressure datum point, with spatial filtering

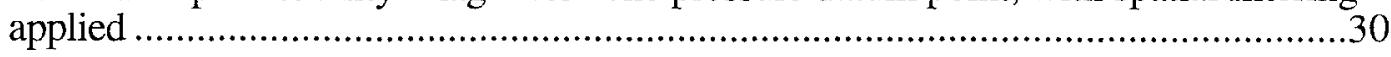

Figure 25. Permeability image calculated from approximately 75 pressure datum points. With no spatial filtering applied, this inverse solution was unstable .............................31 


\section{Adaptive Forward-Inverse Modeling of Reservoir Fluids Away from Wellbores}

\section{Preface:}

This is a report of findings submitted to the Prediction Team of the DeepLook Oil and Service Industry Group by the Lawrence Livermore National Laboratory (LLNL) as deliverables for the Phase I project, entitled "Adaptive Forward-Inverse Modeling of Reservoir Fluids Away from Wellbores". The deliverables include: a report of results, listing(s) of commands that construct and execute codes for selected test problems, and an evaluation with recommendations regarding continuance of this project, including considerations of possible extensions to 3-D and additional technical scope.

\section{Executive Summary:}

This Final Report contains the deliverables of the DeepLook Phase I project entitled, "Adaptive Forward-Inverse Modeling of Reservoir Fluids Away from Wellbores". The deliverables are: (i) a description of 2-D test problem results, analyses, and technical descriptions of the techniques used, (ii) a listing of program setup commands that construct and execute the codes for selected test problems (these commands are in mathematical terminology, which reinforces technical descriptions in the text), and (iii) an evaluation and recommendation regarding continuance of this project, including considerations of possible extensions to 3-D codes, additional technical scope, and budget for the out-years.

The far-market objective in this project is to develop advanced technologies that can help locate and enhance the recovery of oil from heterogeneous rock formations. The specific technical objective in Phase I was to develop proof-of-concept of new forward and inverse (F-I) modeling techniques [Gelinas et al, 1998] that seek to enhance estimates (images) of formation permeability distributions and fluid motion away from wellbore volumes. This goes to the heart of improving industry's ability to jointly image reservoir permeability and flow predictions of trapped and recovered oil versus time. The estimation of formation permeability away from borehole measurements is an 'inverse' problem. It is an inseparable part of modeling fluid flows throughout the reservoir in efforts to increase the efficiency of oil recovery at minimum cost. Classic issues of non-uniqueness, mathematical instability, noise effects, and inadequate numerical solution techniques have historically impeded progress in reservoir parameter estimations. Because information pertaining to fluid and rock properties is always sampled sparsely by wellbore measurements, a successful method for interpolating permeability and fluid data between the measurements must be: (i) physics-based, (ii) conditioned by signal-processing tenets, and (iii) solved with sufficiently rigorous mathematical and numerical techniques. Such a methodology is applied in this project, as we extend the F-I modeling methods developed at LLNL for ground water remediation to DeepLook reservoir problems involving transient multiphase flows. The results obtained at this juncturc are cncouraging; and the proposed objectives of Phase I have been achieved.

The text of this report begins in Section III with a statement of the problem being addressed and the practical motivations. It continues with a discussion of the long-standing fundamental difficulties that need to be resolved or mitigated for successful outcomes to be achieved. Alternative stochastic modeling concepts and approaches that have been, or could be, applied in reservoir 
parameter estimation problems are then considered. From that overview discussion it is apparent that the extended F-I approach in this project was purposefully designed to meld stochastic moment equations of fluid physics (Appendix A) with signal-processing techniques (Appendix B). Robust mathematical and numerical solution techniques were necessarily brought forth (Appendix $C$ ) to solve the resulting nonlinear systems of partial differential equations (PDEs). Six developments in computational physics were critical for successful implementation of this integrated F-I approach in Phase I; they included:

- Novel interpolation techniques

- Spatial filtering techniques

- Simultaneous solution of F-I and spatial-filtering PDEs

- F-I model calibration techniques

- Novel PDE solution schema

- Dynamic unstructured adaptive-grid PDE solution methods

The adaptive-grid PDE solution algorithms and code-generation capabilities that were used in this work are commercially available in a toolkil known as FlexPDE [Nelson,1998, and Backstrom, 1998]. Pending publication of detailed technical articles in scientific journals, the stochastic theoretical basis and mathematical techniques in this work are addressed briefly in Appendices A and B. A brief description of FlexPDE toolkit features is presented in Appendix C.

Section IV presents results from testing the feasibility of proposed F-I modeling concepts and computational techniques. Example problems used in the feasibility-testing were based upon a transient 2-D black oil model (BOM) from petroleum engineering. Numerous test cases were solved and verified successfully in efforts to address such basic questions as: "What is the best image quality that might be expected from F-I permeability interpolations between wellbore measurements with data sets that range from very sparse to very dense?"; "How to extract the greatest amount of information from sparse data sets for reservoir systems, recognizing that these problems do not generally satisfy linearity and other assumptions needed in conventional Fouriertranform-based signal-processing techniques?"; "How to implement alternative spatial filtering techniques in physical configuration space, in lieu of Fourier-transform techniques?"; and "How to suppress noise in both forward and inverse model simulations?" These issues are critical to successful advances toward end-objectives. To investigate these issues in-depth, test cases wcre posed and solved for both densely and sparsely sampled data --- with and without spatial filtering constraints applied. Results are summarized as follows:

\section{Forward Model Verifications}

- Benchmarks and verifications were established for: BOM formulations, FlexPDE algorithms, and forward model simulations of fluid pressure and saturations.

- Two example problems were developed from petroleum engineering for ground-truth verifications. One was a water-flood scenario on a 2-D horizontal domain, and the other was an oil-dome scenario on a 2-D vertical domain. 'Measured' data were abstracted from ground-truth solutions at discrete locations and used as datum points in test cases. 
- Accuracy of forward model solutions was demonstrated to be important in the F-I modeling approach because inverse solutions of permeability are sensitive to not only fluid pressure and saturation but also their gradients.

- FlexPDE solutions resolved the evolution of sharp, disparate pressure and saturation fronts extremely well over the entire problem domain, including local injection/extraction wells. Resolution of evolving fronts is a significant determinant of predicted times, paths, and rates of fluids arriving at production wells, versus remaining in the formation, over time.

- Forward model solutions are additionally important in these F-I techniques because their dependent variables (pressure and saturation) are 'signals' that carry the information from which permeability distributions are determined in inverse PDE solutions.

\section{Inverse Model Verifications}

The resolution of permeability images is a nebulous function of data sparsity. Test cases were thus constructed to span a broad spectrum of data density/sparsity. We examined the relative amounts of information that could be retrieved in F-I images from varying amounts of finitely sampled data. Results are presented (Section IV) for data sets ranging from the most dense sampling (>200,000 datum points) to the most sparse sampling (one datum point) of pressure signals.

\section{Densely Sampled Cases:}

- High-quality images of permeability were produced from densely sampled pressure data (typically thousands to hundreds of thousands of datum points). Results demonstrated that considerable resolving power is attainable with the F-I techniques and FlexPDE solution algorithms. In addition these results provided benchmarks for examining the significance of data sparsity, which is always a central issue in practical reservoir applications.

- Inverse solutions with hundreds of thousands of 'measurements' (Section IV) support the fact that images from a finite number of datum points cannot be truly perfect (unique) -no matter how densely they're spaced -- due to absent information between datum points.

- A suitable level of spatial filtering was generally beneficial for image quality, even when very densely sampled data were tested.

- Effective application of spatial filtering in the F-I method was subject to problem-specific factors, including: data density/sparsity, permeability contrast ratios (at formation interfaces), information propagation modes, and numerical algorithm robustness.

\section{Sparsely Sampled Cases:}

- With Spatial Filtering:

- F-I solutions of permeability were stable with spatial filtering applied effectively, regardless of data sparsity. Results are presented for cases with 1,20,75, and 
300 pressure datum points in the oil-dome example. These results fairly represent countless other cases that were solved with different data sets.

- The permeability images obtained in all cases were plausible representations of ground-truth, commensurate with data sparsity. Image resolution improved progressively as the number of datum points increased, consistent with original expectations.

- The amount of information contained in images from relatively small numbers of datum points (5-20) sometimes exceeded original expectations, which were based on elementary data-sampling criteria.

- No Spatial Filtering:

- F-I solutions of permeability were unstable when pressure data was too sparse, as is true in many inverse techniques.

- Unstable solutions resulted when fewer than 75 'measurcd' pressure datum points were tested in the oil-dome example. This is a dramatic departure from the stable F-I images that were obtained with spatial filtering applied.

It is apparent in Phase I results, and in previous ground water results (Appendices A and B), that the combined signal-processing and physics-based F-I techniques, solved with rigorous mathematical and numerical methods, produce credible images of permeability away from wellbores, commensurate with the available data. In some instances, where many other existing techniques would be manifestly unstable, the F-I method produced surprisingly good images from sparse data. Insights gained in Phase I efforts suggest that additional advances may matcrialize as these principles and solution techniques are developed more fully over time. For example, data sets observed at specified times (snapshots) during wellfield operations may serve as additional constraints for enhancing permeability images. Forward model results further suggested that oil recovery depends on formation permeability texture and contrast ratios, physical scale and shapes of rock heterogeneity, and disparate-scale pressure and saturation gradient evolution. A capacity to better resolve these sensitive features with data from multiple snapshots potentially opens promising new paths toward attainment of far-market objectives.

Section V presents recommendations regarding continuation of this project. From a critical evaluation of Phase I results, we recommend continuation of this project to a Phase II effort. The objective in Phase II would be to further expand the concepts and methods tested in Phase I by including: (i) representative capillary pressure curves and other relevant reservoir data, (ii) further exploration of the practical effects of undersampling on image resolution in more realistic physics models, and (iii) incorporating time-dependent production data with other transient data. We believe that it is important at this stage of development to rule out possible show-stoppers in three essential areas before expending the much greater levels of effort that would be required with 3-D codes and additional technical scope in a final Phase III: First, more general representations of capillary physics may extend the range and possible severity of nonlinear coupling and uncertainty effects that need to be assessed. Second, the nature and significance of image distortion between wellbores must be assessed for a sizeable range of small-to-large data sets for practical wellfield circumstances. With favorable outcomes, these results may provide an early view of the value of individual pressure measurements and the significance of their number and locations relative to 
production and/or injection wells. Third, the inversion techniques applied for steady-state ground water problems (Appendix B) will extend, theoretically, to inversions of data gathered at multiple times during well-field operations. This is a key concept for economically enhancing image resolution from sparse data, which necds to be confirmed in 2-D, with oil-water physics. Each of these extensions represent formidable tasks that we believe can, and must, be resolved in Phase II, on the path toward achievement of final far-market goals.

\section{Statement of the Problem and Overview:}

To maximize oil production, water may be injected into a reservoir to increase pressure gradients that can drive additional oil to wells in successful designs. For greatest success, fluid fronts must remain stable and move oil along the most advantageous pathways via optimal injection and production well configurations. If the front(s) become unstable, injected fluids can break through, leave too much oil behind, and possibly spoil the condition of the reservoir for future recovery efforts. Similarly, if the most productive locations and pathways for water-drive are not identified, maximum recovery may not be achieved. Knowledge of such regions during initial production and prior to break-through could greatly increase total recovery through a more effective strategy for in-field drilling and production operations. A key element in these problems is that reservoirs are usually deep beneath the surface, where moving fluid fronts and essential subsurface properties that detcrmine flow paths cannot be obscrved dircetly and must be estimated betwecn borehole locations.

In efforts to critically determine subsurface behavior, one can study how energy and/or mass is propagated through the system. This may be done by specifying how varying quantities within the system (temperature, pressure, fluid velocity, and saturations) change as functions of time. Such varying quantities may be viewed as signals because they measure excitations and responses of the system. Signals play a dual role: First, they are used to describe the performance of reservoir operations, frequently in conjunction with forward models that forecast transient flow behavior and production of fluids from wells in the system. Second, signals carry information that is needed in inverse problems to determine rock properties, as well as the interactions among various fluid and formation features in the completc rescrvoir. This dual role of signals suggests that interpolations of pressure and rock properties between wellbores involves a circular modeling process with forward and inverse flow solutions. On the one hand, interpolations of pressure data must satisfy a forward mass balance equation, which depends upon values for spatially-distributed rock properties (porosity and absolute permeability). On the other hand, inverse models are solved for values of absolute permeability between wellbores from interpolated pressure and pressure-gradients, which must ultimately satisfy the forward mass balance equation and measured data. An iterative forwardinverse (F-I) process, alone, achieves mutually consistent interpolations of pressure, pressuregradients, saturations, and permeabiltiy that finally satisfy both the forward and inverse flow equations. All of the interpolated variables must additionally respect signal-processing principles for sparse sampling effects. In other words, the forward, inverse, and signal modeling processes are fundamentally inseparable. Hence advances in F-I modeling will play a potentially pervasive role in optimization of monitoring strategies, estimation of formation properties from sparse data, flow predictions, uncertainty/sensitivity analyses, and re-evaluations of the subsurface behavior consistent with signal theory as new data are obtained. 
Circular modeling, inverse modeling, and history-matching are variously related methods for estimating porous media properties and fluid behavior between measurements in wellbores. They all address a critical technical obstacle that confronts every subsurface project: Interpolations between borehole data are non-unique; and existing parameter estimation methods are constrained either weakly by, or at variance with, essential laws of physics, mathematics, and signal-processing principles between datum points. Such parameter estimation problems are clearly ill-posed because material properties can never be sampled and measured perfectly, at any physical scale. So physical properties deduced from finitely sampled data (e.g., for fluid distribution, pressure, permeability, porosity) will always be distorted to some degree, which makes predictions of future flow behavior, production yields, and costs subject to more or less uncertainty. The fact that an indefinite number of admissible parameter realizations generally exists for discretely sampled data does not mean that subsurface parameter estimation is meaningless. It indicates that well-founded and well-executed concepts of physics, mathematics, and signalprocessing are needed to extract maximum information from the available data. The techniques used should consider both hard and soft data; and they should ultimately encompass sensitivity coefficients, best solutions, and suitable measures of uncertainty propagation.

In addition to manual history-matching methods, numerous deterministic and/or statisticallybased inverse theories and numerical algorithms (codes) have been advanced over the past twentyfive years. A common thread is that all of the methods have significant shortcomings at this time. For more detailed descriptions of individual inverse methods, one can consult numerous review articles, text books, and surveys [Zimmerman et al, 1998; McGlauglin and Townley, 1996; Carle and Fogg, 1996 a and b; Sun, 1994; Newman and Orr, 1993; Gelhar, 1993; Dagan, 1989; Carrerra and Newman, 1986a; Yeh, 1986; and Beran, 1968]. We therefore reviewed at the outset of Phase I the full backdrop of physics principles and inverse solution techniques that have been applied in porous media flow problems. Noted immediately was the fact that, although physical systems sometimes behave deterministically, they are nonetheless stochastic in their basic nature because fundamental uncertainties exist at all scales of measurement. Hence both dynamics and statistics must be considered in order to extract essential information from measured data.

To revicw the fundamental basis of stochastic modeling one looks to kinctic theory, where dynamical and statistical axioms of physics are used to generate continuum flow equations of mass, momentum, and energy balance. Two theoretically equivalent approaches are found: one is a random field approach, and the other is represented by a heirarchy of statistically averaged moment PDEs $^{1}$. A moment PDE approach is used in this project because, among other factors, new PDE software tools have recently become available to help resolve classic long-standing difficulties associated with non-uniqueness, noise, nonlinearity, mathematical instability, and inadequate numerical PDE solution methods that previously thwarted progress in moment PDE approaches ${ }^{2}$. The physics and mathematical techniques developed in this F-I modeling approach may also serve well in several other existing stochastic and least-squares methods, as well as in manual historymatching approaches to parameter estimation.

\footnotetext{
' Both quantum and classical mechanical representations have been applied to analyze stochastic systems in diverse technical problems for several decades, as discussed and referenced in the Theory section that is presented in Appendix A.

${ }^{2}$ Interested readers may wish to peruse the Theory section in Appendix A to examine other factors that presently recommend consideration of the moment PDE approach.
} 
FlexPDE and PDEase [Nelson,1998, and Backstrom, 1994,1998] are two commercially available toolkits that provide state-of-the-art numerical PDE solution techniques and automated code-writing capabilities for solving diverse scientific problems. They enable general practitioners to solve the nonlinear PDE systems that arise in F-I reservoir modeling, where the underlying assumptions of alternative Fourier-analysis-based inverse and signal-processing techniques do not generally apply. These toolkits have yielded high levels of accuracy and work-saving economies in the development of this F-I modeling methodology by their effective utilization of novel codegeneration software. Some of the most robust adaptive-grid PDE solution methods that have emerged in applied mathematics research over the past twenty five years are the "engines" in these toolkits. (Additional applications of PDEase and FlexPDE in diverse disciplines can be seen in the text by Backstrom [1998].) The FlexPDE toolkit by Nelson [1998] has been used to solve all of the examples presented in this report. Looking ahead, extensions to 3-D reservoir modeling in the out-years will be paced by the performance of recently released 3-D FlexPDE software.

A formidable problem in parameter estimation techniques is the need to effectively manage noise effects. Noise in stochastic simulations may correspond to both physical and non-physical sources. Physical noise sources would be associated with natural fluctuation phenomena in the flow system, per se, and with physical measurement processes. Non-physical noise sources would include interpolations of sparsely sampled data, faulty conceptual models, and numerical errors in forward and inverse model simulations. Numerical PDE solution errors and faulty concepts are problematic in model simulations because they frequently cannot be distinguished from physical effects. They may then debilitate one's capacity to deal effectively with the legitimate problems posed by non-uniqueness and mathematical instability. It was thus important to identify and eliminate at the outset not only such non-physical noise sources as numerical PDE solution errors but also failures to respect signal-processing principles for sparsely sampled data. The practical working principle being applied here is that the space of admissible, mutually-consistent permeability and pressure realizations is expected to shrink as increasingly restrictive constraints, including elimination of non-physical noise and other modeling artifacts, are used to condition parameter realizations. Some key advances by Gelinas et al [1998, and this report] enabled the F-I methods to apply this working principle productively in Phase I. Included in these advances are:

- Novel interpolation techniques:

A modified finite element method was constrained to agree with wellbore measurements while solving forward and inverse flow equations. Resulting flow solutions respect the discrete wellbore data and are, moreover, intrinsic physics-based interpolators of pressure and permeability between wellbores.

\section{- Spatial filtering techniques:}

Flow data interpolations must be filtered (smoothed) so that their spatial frequency spectra are consistent with discrete sampling principles [Bracewell, 1986; McGillem and Cooper, 1984]. Ancillary PDEs were developed to spatially filter both pressure and pressure-gradients in physical configuration space consistently with forward and inverse PDE solutions. This technique enables one to effectively calibrate nonlinear continuum flow solutions (interpolations) with discretely sampled data. 
- Simultaneous solution of F-I and spatial-filtering PDEs:

Nonlinear systems of forward and inverse flow PDEs were solved simultaneously with spatial-filtering PDEs in order to both suppress noise and help mitigate mathematical instability.

- F-I model calibration techniques:

The inverse flow equation was solved and calibrated with permeability data according to rigorous Cauchy criteria, which are otherwise violated in many parameter estimation techniques.

- Novel PDE solution schema:

Continuum mappings of flow characteristics were solved from pressure data with a novel PDE scheme, in lieu of calculating discrete characteristics from ordinary differential equations (ODEs) that do not fill the physical domain. This schema facilitates rigorous calibrations of inverse PDE solutions according to Cauchy criteria.

\section{- Dynamic unstructured adaptive-grid PDE solution methods:}

Numerical errors and other modeling artifacts were reduced markedly by using dynamic unstructured adaptive-grid methods to solve both signal-processing and F-I flow PDEs. This improves the resolution of both permeability images (inverse solutions) and flow images (forward solutions) on a self-consistent basis.

These composite F-I modeling techniques help mitigate the classic perils posed by mathematical instability, noise, and non-uniqueness. To execute these techniques successfully, it was necessary to employ the FlexPDE toolkit [Nelson,1998, and Backstrom, 1998]. Pending publication of detailed technical articles in scientific journals, the stochastic theoretical basis and mathematical techniques applied in this work are addressed briefly in Appendices A and B. A brief description of FlexPDE toolkit features is presented in Appendix C. Such key aspects of parameter estimation as 3-D simulations, uncertainty propagation, sensitivity coefficients, "best solution" techniques, etc., were not included within the scope and resources of Phase I. There appears to be considerable promise, however, for significant new advances to be made in some of these omitted topics in the near future, as a natural extension of the F-I modeling and PDE solution techniques used in the present work.

\section{Examples and Results}

This section examines results of numerous test cases that were posed and solved in terms of a black oil model (BOM) for oil-water systems from petroleum engineering. The BOM represents a two-phase (oil-water) immiscible, incompressible flow system, with zero capillary pressure. It provides the conceptual model basis for two example problems that are used in this section to assess the feasibility of the F-I modeling concepts and mathematical techniques. The example problems were constructed to have ground-truth solutions for verification purposes. One example problem is an idealized water-flood scenario on a 2-D horizontal rectangular domain with zero gravitational forces; and the other is a 2-D vertical oil-dome water-driven model with gravitational forces. The flow systems in all cases are described by the mean flow equation, with stochastic 
residual flux terms taken to be negligible. Residual fluxes are second-order statistical fluctuation terms that contribute to the rate of change of the dependent variable in the mean flow equation. They act like other source terms that generally appear in the mean flow equation. Their neglect is not a serious limitation in proof-of-concept assessments at this stage of development. (Additional implications pertaining to this assumption are discussed in Appendix A; and a detailed development of the BOM is presented in Appendix E.)

Dynamic adaptive-grid PDE solution techniques in the FlexPDE toolkit facilitate problem verifications. They enable users to arbitrarily specify a PDE solution accuracy that is to be achieved in each problem run. The PDE solver adapts unstructured spatial and temporal numerical grids automatically in an iterative numerical solution process. Numerical integration cycles are executed in FlexPDE until both global and local accuracy criteria are satisfied internally. If a user-specified accuracy goal is not attained, a code run will usually not terminate gracefully. But in all instances a record of diagnostic information reports the state and progress of the numerical solution process during each attempted run. The combination of such accuracy-controlled adaptive-gridding methods with informative run-time performance diagnostics accelerates test case verifications, largely because failures of the PDE solver to deliver demanded accuracy (within practical limits) will usually lead quickly to the identification of inappropriate concepts, mathematical errors, inconsistent data, inadequate or misapplied algorithms, or other sources of error. To practically verify that PDE solutions have 'converged', several runs are typically executed with progressively greater accuracy demanded in each successive run, until the solutions are deemed to have approached a 'true solution', or not. If code changes, including entirely new conceptual or mathematical models are required, the automated code-writing features in FlexPDE readily produce new codes, which further facilitate code and test-problem verifications.

\section{IV-1 Detailed Descriptions of Example Problems and Results}

\section{Example Problem 1: IIorizontal (2-D) water-drive problem.}

\section{Forward Model Verifications}

The first example problem (Figure 1) simulates an idealized water-flood scenario on a 2-D horizontal rectangular domain, with zero gravitational forces. This problem was also considered in a recent article by Saad and Zhang [1998]. The BOM formulation in equations (E-1) - (E-9) of Appendix E was used in Saad and Zhang's work, and in our work here. Since the permeability is constant in this example, the inverse solution for $\mathrm{K}$ is trivial. So the objective in this example was to benchmark the dynamic adaptive-grid finite element solution techniques in FlexPDE against a current alternative adaptive-grid method, such as the one applied in the IMPES (Implicit PressureExplicit Saturation) approach [Aziz and Settari, 1983] by Saad and Zhang [1998]. 


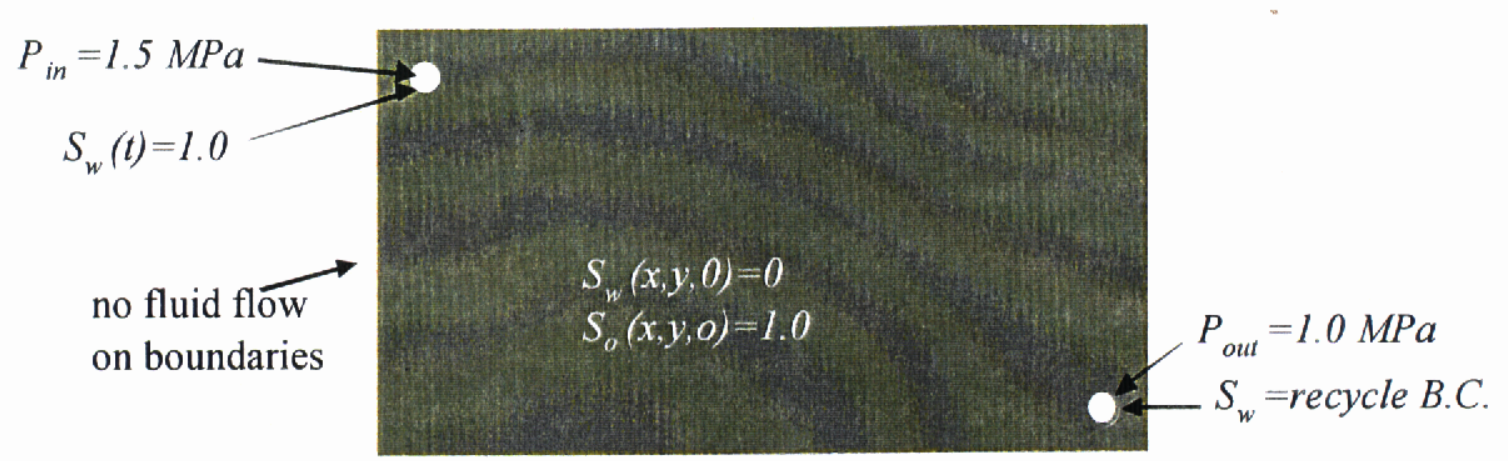

Figure 1 - 2-D horizontal oil production water injection model

It is worthwhile to briefly point out some of the immediate contrasts and similarities between these respective approaches. In Saad and Zhang's work the pressure equation was discretized using a finite volume method; and the resulting linear system of algebraic equations in the pressure variable (for the latest calculated saturation variable) was solved by a bi-gradient-like method. For the saturation equation, they use a modified and robust upwind scheme (following the MUSCL approach, an acronym for Monotonically Upstream-centered Scheme for Conservation Laws) that seeks to reduce the diffusive effect that is intrinsic in rudimentary upwinding procedures [Van Leer, 1977; Randall, 1990].

By way of contrast, the FlexPDE toolkit used in the F-I approach solves the pressure (P) and saturation (S) equations simultaneously, rather than sequentially. The dynamic adaptive-grid solution algorithms incorporate finite element Galerkin methods of order 2 or 3 on the spatial domain. The temporal discretization is a fully implicit BCE (Backward Cauchy-Euler) method. As a result of solving in $\mathrm{P}$ and $\mathrm{S}$ simultaneously, the discretized equations generated with the Galerkin technique obviously form a nonlinear system of algebraic equations. In order to solve the nonlinear algebraic system, it is first linearized using a variant of the Newton-Raphson method. With today's robust solvers for linearized algebraic systems, many challenging nonlinear PDE systems can be solved accurately. But there is a price to be paid, software-wise. Each different type of PDE (elliptic, hyperbolic, linear, nonlinear, mixed types, etc.) can present a host of demands, of widelyvarying severity, upon solvers of the resulting algebraic systems. So numerous matrix solution options are made available to users of general-purpose PDE toolkits. From the several solution options available in the FlexPDE toolkit, we found that a variant of Lanczos' method was sufficient for solving simultaneous equations for $\mathrm{S}$ and $\mathrm{P}$ in the present example [Lanczos, 1997]. An interesting finding was that, although the time discretization in FlexPDE is only first-order accurate, it nonetheless seems to provide very satisfactory answers in the reservoir problems addressed so far. This result was satisfying in view of the numerical difficulties that might have been anticipated in solving the time-evolving saturation equation simultaneously with the instantly-equilibrated pressure equation. We note further that it was important to stabilize the saturation equation in the present approach by adding a regularizing term, $\varepsilon \Delta S$, to equation (E-21). (The notation, $\Delta S$, represents the Laplacian of the water saturation). Values of $\varepsilon$ are made sufficiently small to retain the basic integrity of the original saturation PDE. Finally, the numerical approaches in both Saad's work and the F-I techniques refine and merge grid cells according to criteria based on various internal estimates of local truncation error, as well as global measures. 
The following numerical values, in mks units, are assigned to reservoir parameters in this example problem:

$$
\begin{aligned}
& \mathrm{K}=5 \times 10^{-13} \mathrm{~m}^{2}, \quad \text { (absolute permeability) } \\
& \varphi=.33, \quad \text { (porosity) } \\
& \mu_{\mathrm{w}}=1 \times 10^{-3} \text { Pa.s, } \quad \text { (water viscosity) } \\
& \mu_{0}=4 \times 10^{-3} \text { Pa.s. (oil viscosity) }
\end{aligned}
$$

The domain $\Omega$ is taken formally to be the rectangular region (Figure 1),

$\Omega=\{(\mathrm{x}, \mathrm{y}): 0 \leq \mathrm{x} \leq 100 \mathrm{~m}, 0 \leq \mathrm{y} \leq 50 \mathrm{~m}\}$.

A water-injection well with an effective radius of 0.5 meter is located near the north-west corner of the domain and is centered at $(6.25,43.75 \mathrm{~m})$. An extraction well of the same radius is placed, in similar fashion, near the opposite (south-east) corner. Although the size of the wellbore may seem to be large, it has no major negative repercussions when modeling at field scales, especially away from the well location. (It is often possible to adjust the pressure BCs fairly accurately in going from one well size to a concentric and relatively larger size.) Simulations that use the actual well size will tend to over-grid unnecessarily in the vicinity of the well, unless, of course, one wishes to simulate flow behavior within the immediate wellbore neighborhood, possibly including flow within the wellbore itself. The approach followed for field-scale simulations in the present work simply excludes the region interior to each well from the definition of $\Omega$. In this way, the boundary of $\Omega$ consists of exactly three closed curves: the rectangle perimeter, $\Gamma_{1}$, and the injecting and extracting well circumferences, $\Gamma_{2}$ and $\Gamma_{3}$, respectively. The boundary $\Gamma_{1}$ is assumed to be impermeable. That is, the outward normal component of the flow velocity, $\mathrm{V} \cdot \mathrm{n}$, vanishes over $\Gamma_{1}$, which translates to the pressure $\mathrm{BC}, \partial \mathrm{P} / \partial \mathrm{n}=0$, where $\mathrm{n}$ is the outward normal vector to $\Gamma_{1}$. Pressure values of 1.5 and $1 \mathrm{MPa}$ are assigned on $\Gamma_{2}$ and $\Gamma_{3}$, respectively. Also notice that, because the problem of determining the pressure is only a BVP, these boundary assignments are sufficient to solve for $\mathrm{P}$ at any instant in time.

In solving the saturation equation (E-21), the initial saturation, as well as the saturation specification along some appropriate Cauchy curve for all $t>0$, must be known. It can be shown that, in this case, the curve $\Gamma_{2}$ in Figure 1 is an admissible Cauchy curve because: (i) the saturation is always maintained at the prescribed value of 1.0 for all $t>0$, and (ii) less trivially, all characteristic curves emanating from $\Gamma_{2}$ span the entire domain, $\Omega$. On $\Gamma_{1}$, the boundary integrals for the saturation equation can still be set to zero, because its integrand is a multiple of $V \bullet n$, where $V$ is the velocity and $\mathrm{n}$ is an outward normal. At the extraction well, however, the boundary condition is actually a part of the solution process, which means that no boundary specification is required there. The FlexPDE toolkit has a provision that enables users to implement the 'no-specification' boundary condition according to the finite element 'recycle' techniques that were discussed, for instance, by Oden et al [1986]. This option requires the integrands of certain boundary integrals to be provided by the user in the FlexPDE problem setup commands (Appendix D).

The initial and boundary conditions for this example are summarized as follows:

Initial conditions, $\mathrm{t}=0$ :

$$
\mathrm{S}=0 \text { on } \Omega \text {, }
$$


$\mathrm{P}=\mathrm{P}_{0}$, where $\mathrm{P}_{0}$ is the solution of equation (E-22), with $\mathrm{S}=0$ and the pressure $\mathrm{BCs}$ listed next.

\section{Boundary conditions:}

On $\Gamma_{1}: \mathrm{V} \cdot \mathrm{n}=\mathrm{fV} \cdot \mathrm{n}=0$ for $\mathrm{t} \geq 0$.

On $\Gamma_{2}: P=1.510^{6} \mathrm{~Pa}$ for $\mathrm{t} \geq 0$, and $\mathrm{S}=1$ for $\mathrm{t}>0$.

On $\Gamma_{3}: \mathrm{P}=10^{6} \mathrm{~Pa}$ for $\mathrm{t} \geq 0$, and a 'no-specification' $\mathrm{BC}$ for $\mathrm{S}$.

Results for this test example are shown in Figures (2) - (7). Figure 2 shows the isocontours of water saturation as a function of $\mathrm{x}$ and $\mathrm{y}$ at $\mathrm{t}=100$ days. The adaptive-grid algorithm in FlexPDE is clearly tracking the water saturation front. The abbreviated text immediately beneath the figure lists the FlexPDE input file name (descriptor file), the current time-step cycle, total elapsed time in seconds, time-step size in seconds, number of spatial grid nodes utilized in this stage, the corresponding number of cells, the RMS error over the entire domain, and the total volume of displaced oil up to this time. Because no injected water has yet reached the extraction well, we confirmed that the volume of displaced oil is equal to the volume of injected water. Figure 3 shows the self-adapting grid that is used at the specific cycle (113) of the FlexPDE solution process. The density of grid nodes is high all along the current location of the moving water saturation front and also near the injection well, where the saturation gradient is steepest. The water and oil saturation gradients are null at the extraction well at this time (100 days); so the cluster of cells in the vicinity of the extraction well is only there for resolving the local pressure gradients of oil extraction and for logical considerations related to the geometry of that region. Figure 4 shows a snapshot of the saturation distribution after 200 days; and Figure 5 displays the first-time arrival of the water saturation front at the extraction well after 340 days. Figure 6 exhibits in greater detail the so-called water break-through in a square with sides equal to eight well-diameters, about the extraction well. Finally, Figure 7 displays the oil production and the production-rate history curves. The latter curve clearly indicates the instant at which the water break-through occurred. The evolution of disparate saturation and pressure gradients, including the fluid fronts and flows near extraction wells (associated with robust outflow boundary conditions) are sharply resolved relative to other numerical solution techniques that are used widely in forward reservoir models. These are obviously significant factors for accurately determining the times, paths, and rates of fluids arriving at prodution wells. They take on additional significance in F-I parameter estimation methods, because inverse PDE solutions are reliant upon the coupled forward model solutions. The accuracy of forward model solutions and derivative pressure gradients is all the more important because forward solutions carry the information from which permeability distributions are determined with the inverse PDEs, as in the second example problem for an oil-dome scenario. 
OW Water-Drive Homogeneous Sandstone

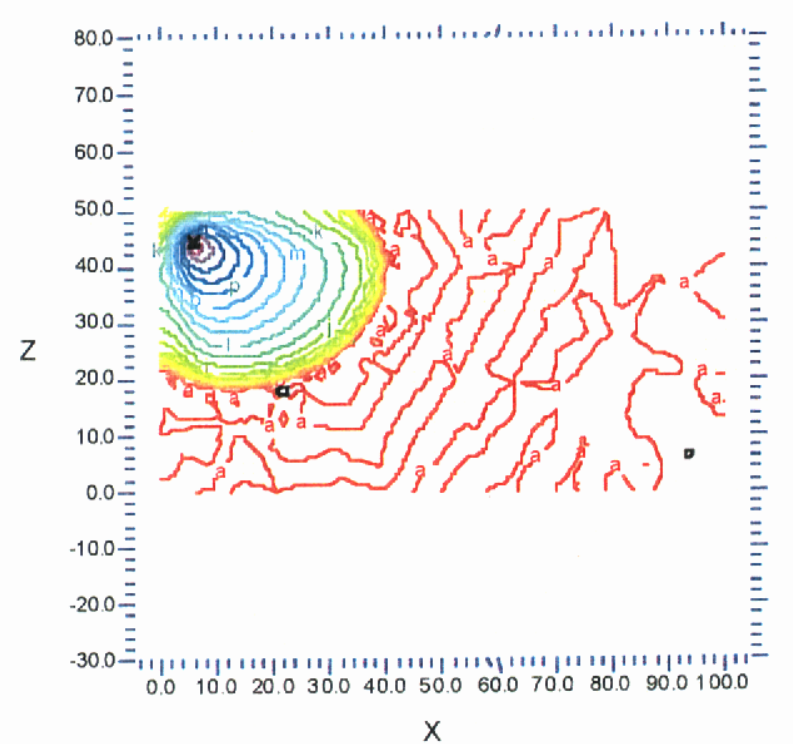

$18: 52: 146 / 24 / 98$

FlexPDE 2.04

SAAD2W1: Cycle $=113$ Time $=8.64 \mathrm{e}+6 \mathrm{dt}=2.4442 \mathrm{e}+5$ p2 2444 Nodes 1183 Cells RMSErr $=0.0019$ Integral $=589.1901$

Figure 2 - Isocontours of water saturation after 100 days

OW Water-Drive Homogeneous Sandstone

$\mathrm{X}$

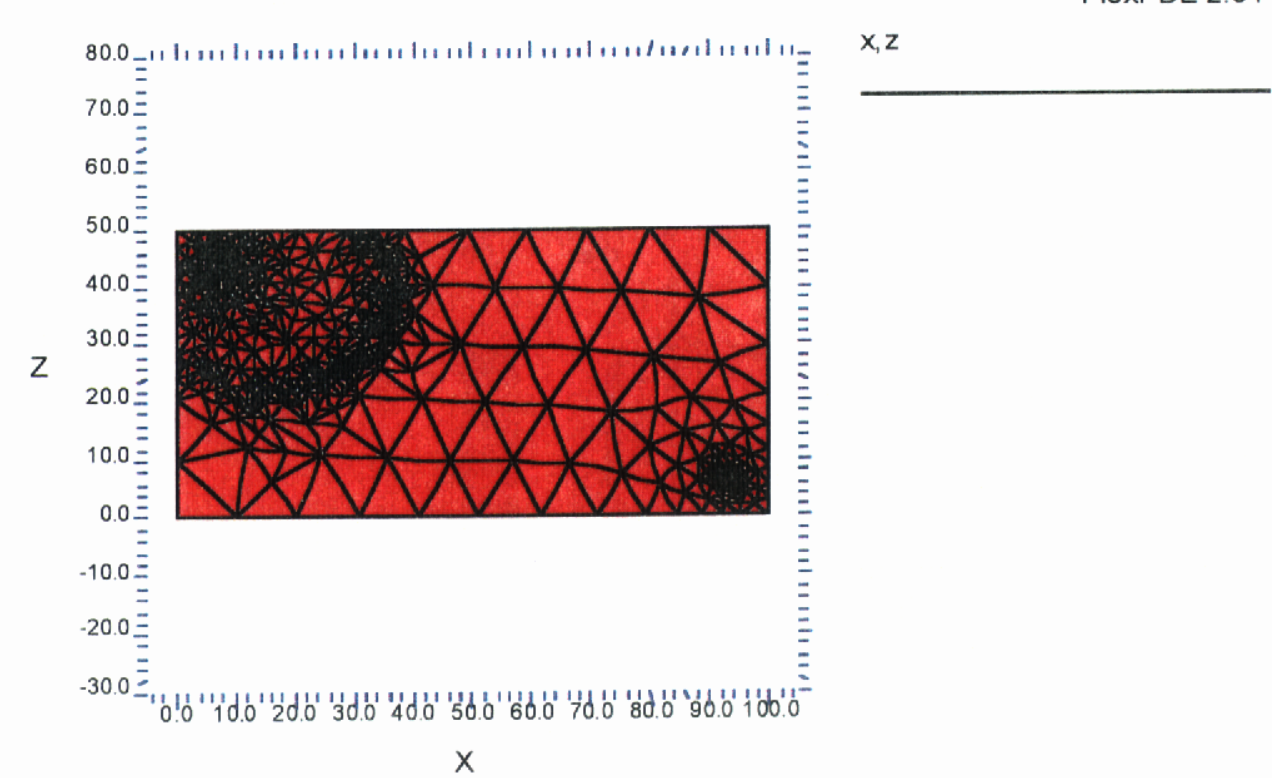

18:52:14 6/24/98

FlexPDE 2.04

SAAD2W1: Cycle $=113$ Time $=8.64 \mathrm{e}+6 \mathrm{dt}=2.4442 \mathrm{e}+5$ p2 2444 Nodes 1183 Cells RMS Err=0.0019

Figure 3 - Corresponding unstructured adaptive grid distribution after 100 days. 
OW Water-Drive Homogeneous Sandstone

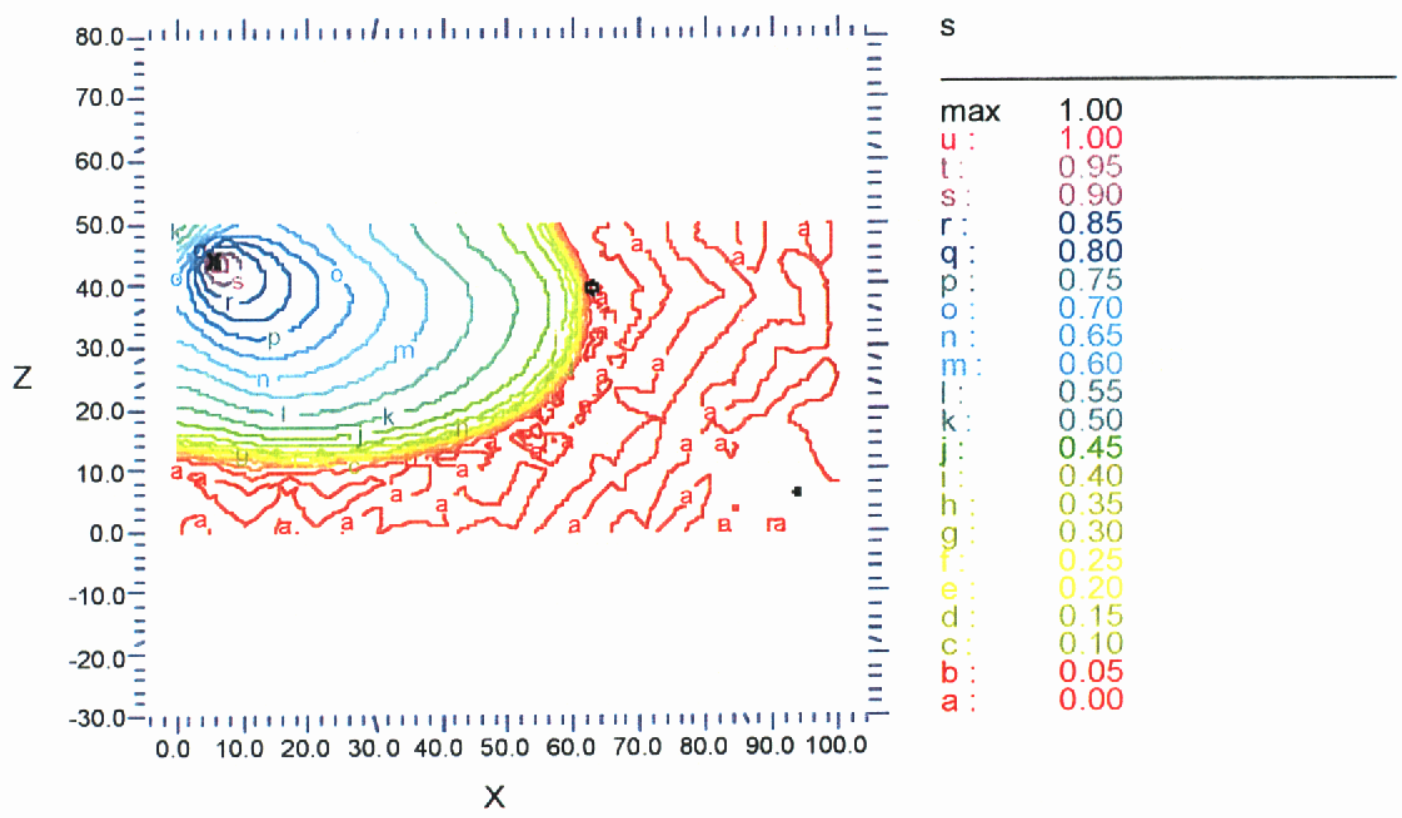

SAAD2W1: Cycle $=147$ Time $=1.728 e+7 \mathrm{dt}=2.939 e+5$ p2 2710 Nodes 1315 Cells RMS Err=0.0016 Integral $=1235.334$

Figure 4 - Isocontours of water saturation after 200 days

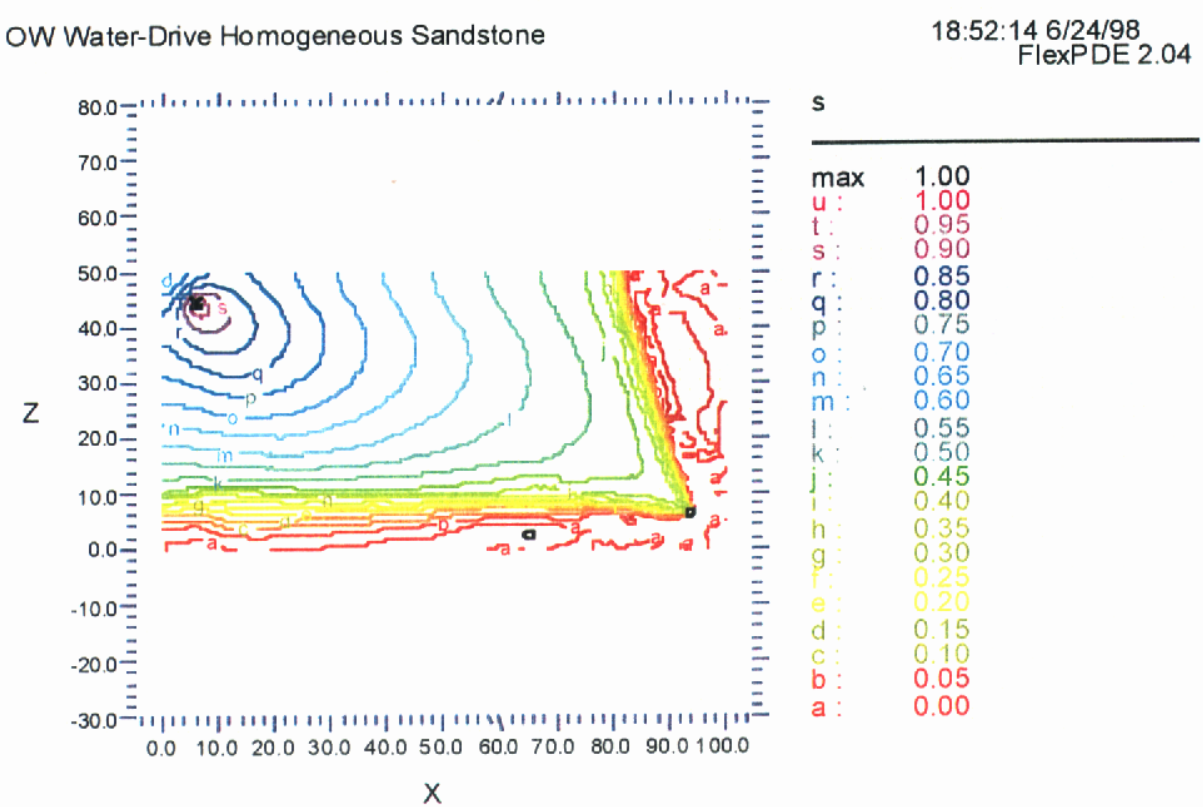

SAAD2W1: Cycle $=206$ Time $=2.9376 \mathrm{e}+7 \mathrm{dt}=2.3333 \mathrm{e}+5$ p2 1909 Nodes 920 Cells RMSEr $=0.0019$ Integral= 2206.552

Figure 5 - Water saturation at break-through after 340 days 
OW Water-Drive Homogeneous Sandstone

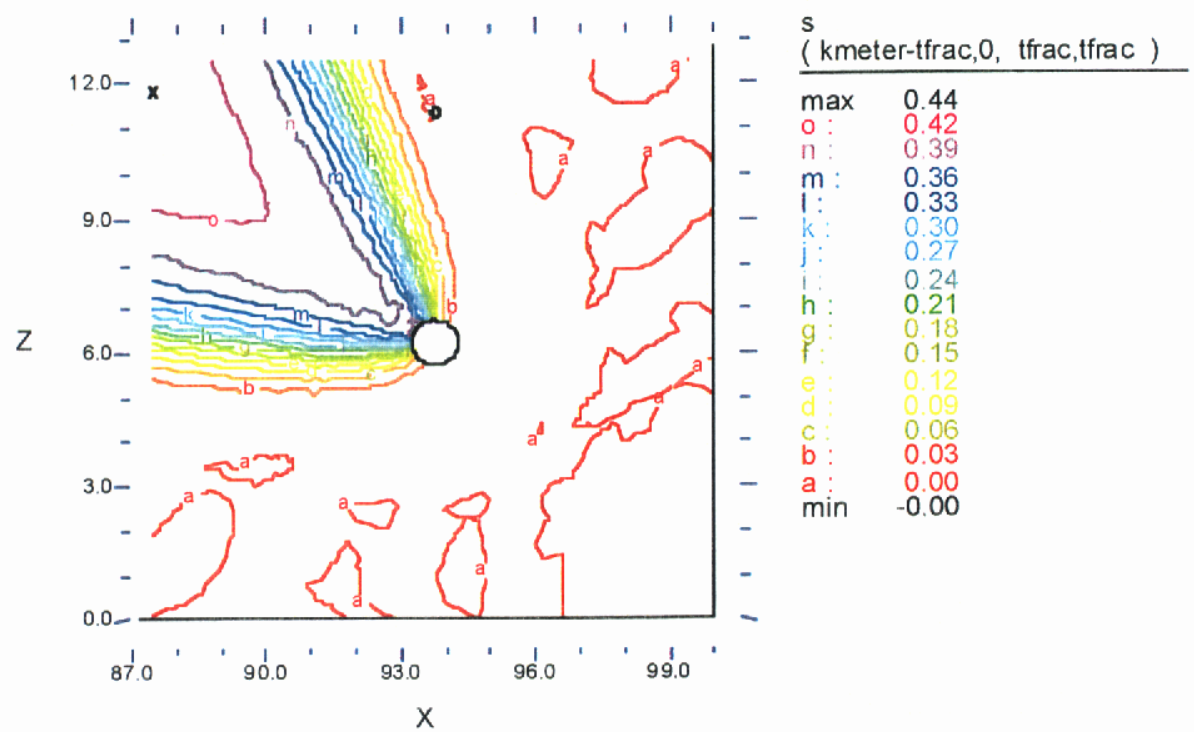

$18: 52: 146 / 24 / 98$

FlexPDE 2.04

SAAD2W1: Cycle $=206$ Time $=2.9376 \mathrm{e}+7 \mathrm{dt}=2.3333 \mathrm{e}+5$ p2 1909 Nodes 920 Cells RMS En $=0.0019$ Integral $=13.53013$

Figure 6-A blow-up of the water saturation front arrival at extraction well (see Figure 4).
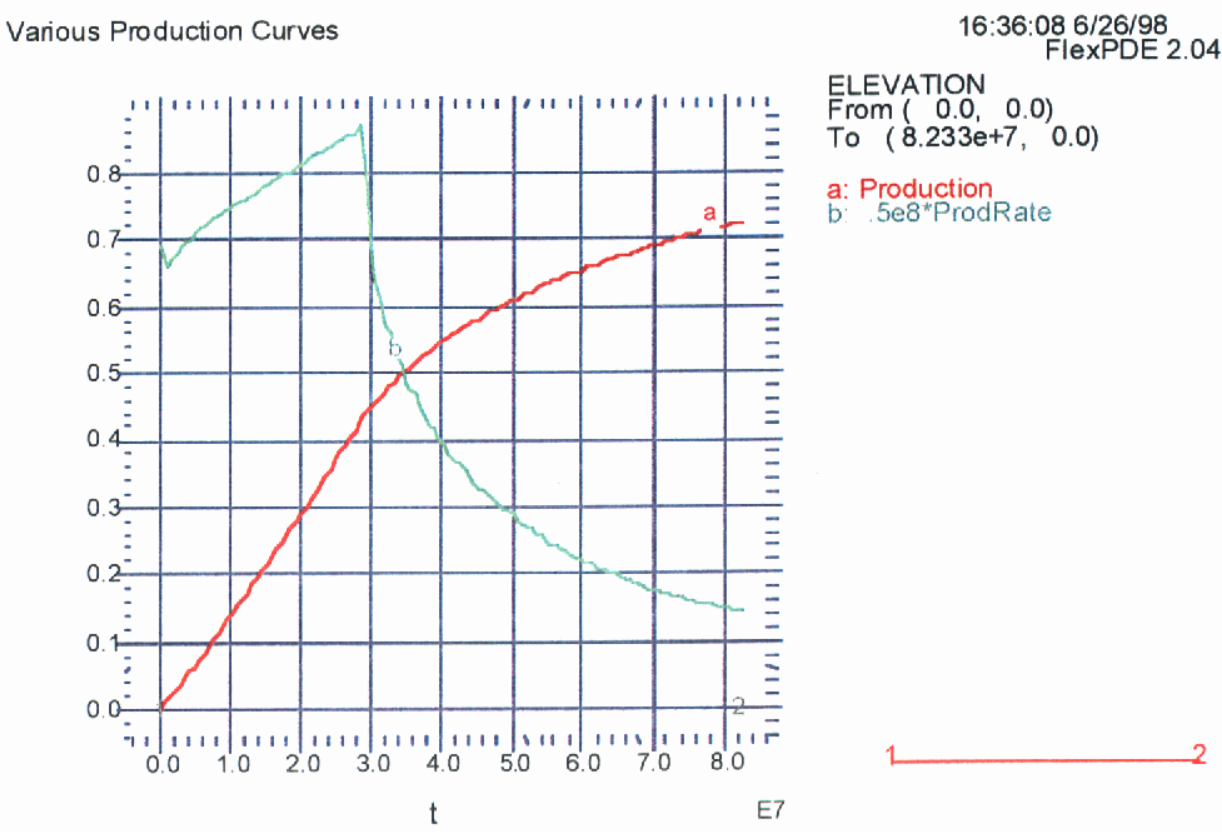

PCURVES: Grid\#4 p2 33993 Nodes 16692 Cells RMSErr $=16720$ Integral $(a)=3.909453 \mathrm{e}+7$ Integral $(\mathrm{b})=3.628479 \mathrm{e}+7$

Figure 7 - Production curve (red) and normalized Production Rate curve (green) calculated at the extraction well from $t=0$ to 1000 days . 


\section{Example Problem 2: A vertical (2-D) oil-dome problem}

This example (Figure 8) considers a vertical 2-D cross-section of an oil-dome that loosely incorporates Pampano-like field features. An extraction well with an assumed down-hole pressure value (maintained by pumping) is placed near the dome crown. Hydrostatic pressure of a supporting aquifer is assigned at both of the vertical sides near the rectangle base. This is the only place where water can flow into the dome. All pressure assignments are assumed to be timeinvariant; and all of the remaining boundaries are assumed to be impermeable.

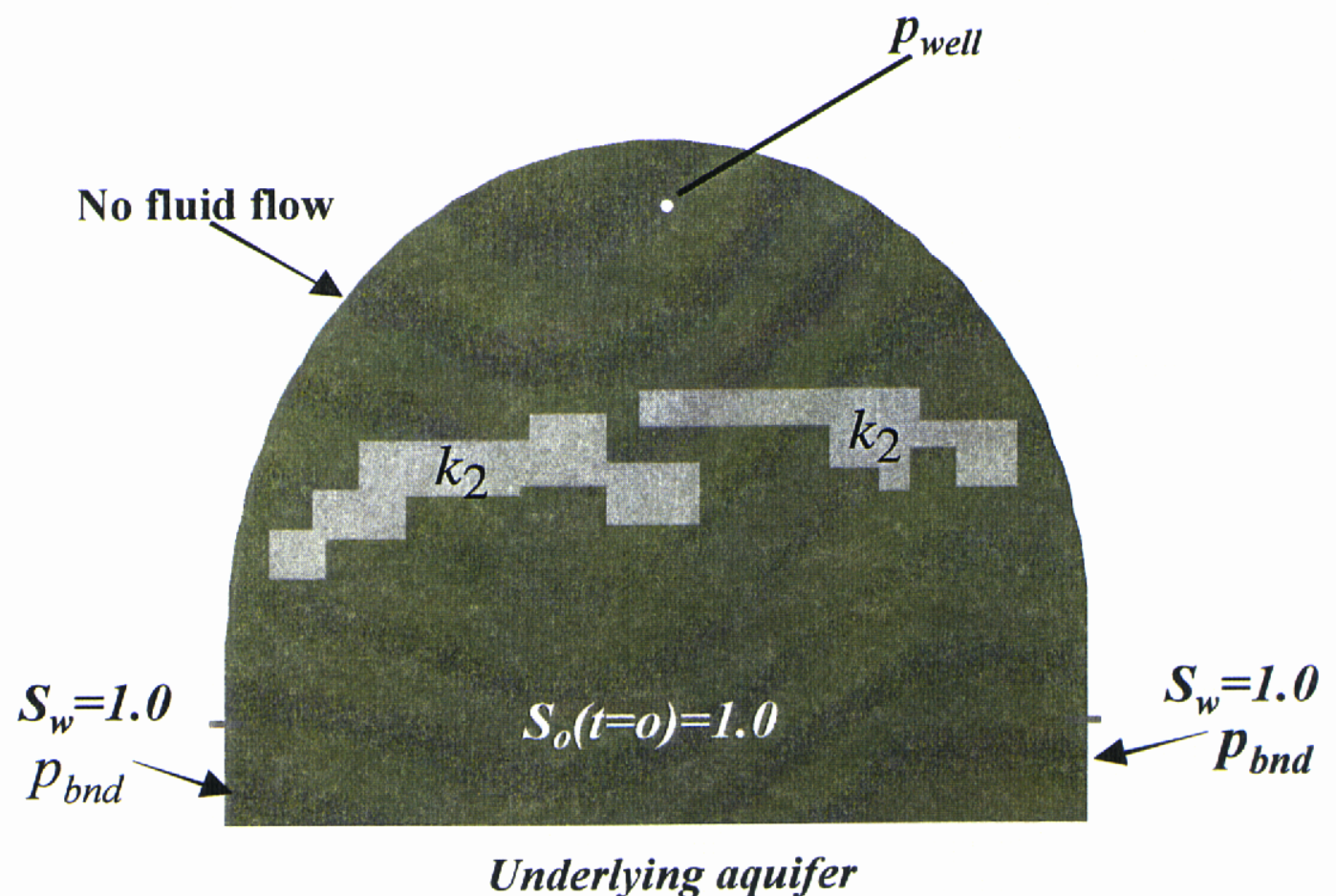

Figure 8-2-D vertical oil production water-drive model

A major objective in this example (addressed in Case 3 below) was to determine the capacity of the F-I modeling method to calculate a known heterogeneous permeability distribution in a reservoir, given sufficiently dense 'measurements' of pressure and permeability. The working principle is: If an inverse method is unable to produce a high-fidelity image when given sufficient data, there is little reason to expect the method to yield credible results for real situations with sparse data. The purpose of Cases 1 and 2 below was to verify that the FlexPDE solution algorithms are capable of resolving sharp saturation fronts and large disparate pressure gradients in forward solutions, as well as sharp interfaces between low- and high-permeability formation substructures. These cases are important because failures to resolve physical gradients adequately in simulations frequently yields erroneous estimates of fluid front propagation in heterogeneous formations, which may in turn yield erroneous estimates of where, and how much oil may be 
remaining over time in well-field design simulations and operational decision-making. (See Figures 9-16.)

The governing PDEs for this example are obtained by including gravitational terms in equations (E-21) and (E-22), which were given previously (Appendix E). Heterogeneity in this simplified dome model is modeled with two saturation-independent permeabilitics: $K_{C}$ represents the permeability of channel sands, and $\mathrm{K}_{\mathrm{I}}$ represents interchannel permeability. Rock and fluid properties, including the quadratic relative permeability, are taken to be the same here as in the previous water-flood example, with $K_{C}=K$. Depending on values of the ratio, $R=K_{I} / K_{C}$, the relative roles of individual physical processes, e.g., entrapment, refraction and retardation, are problem-specific and can significantly affect the transient evolution of recovered and remaining oil over time, as will be evident in the results displayed in Figures (9) - (14) for a case with $\mathrm{R}=1 / 10$ and in Figures (15) and (16) with $R=0$. The results obtained in these two cases clearly indicate the capabilities of our approach in tracking the complex evolution of saturation fronts, as they split, refract, and undergo retardation while propagating through the reservoir.

\section{Forward Model Verifications with Densely Sampled Data Sets}

\section{Case 1: Forward solution for pressure and saturation with $R=1 / 10$}

In this first case, with $\mathrm{R}=1 / 10$, it can be seen in Figures 8 and 9 that water from the supporting aquifer enters the lower vertical sides of the dome and drives oil through portions of the two lowpermeability interchannel sub-structures, as well as through the more permeable channels. Figure 10 indicates how the unstructured numerical solution grid adapts dynamically to anticipate, and follow, the moving saturation fronts simultaneously with pressure gradients near the extraction well. The numerical grid undergoes both refinement near large gradients and un-refinement in parts of the domain that do not contain large gradients. Figures 11 and 12 show the arrival of first water saturation front at the extraction well at 200 days. The saturation front on the right-hand-side of the dome has not yet arrived at the well. Nor has the saturation front yet made its way through the relatively narrow channel between the two low-permeability sub-structures in the central-dome region. The water fronts have however continued to penetrate the outer regions of the low- $\mathrm{K}$ substructures. Figures 13 and 14 show the arrival of the second saturation front at the extraction well at 220 days. The lagging saturation front has started to enter the narrow channel between the two low-K sub-structures. At 420 days we observed that significant oil (approximately 20\%) was retarded in the low-K interchannel sediments. After 4 ycars, cssentially all of the oil in the dome has been produced in this case. 
OW Water-Drive Heterogeneous sand/silt

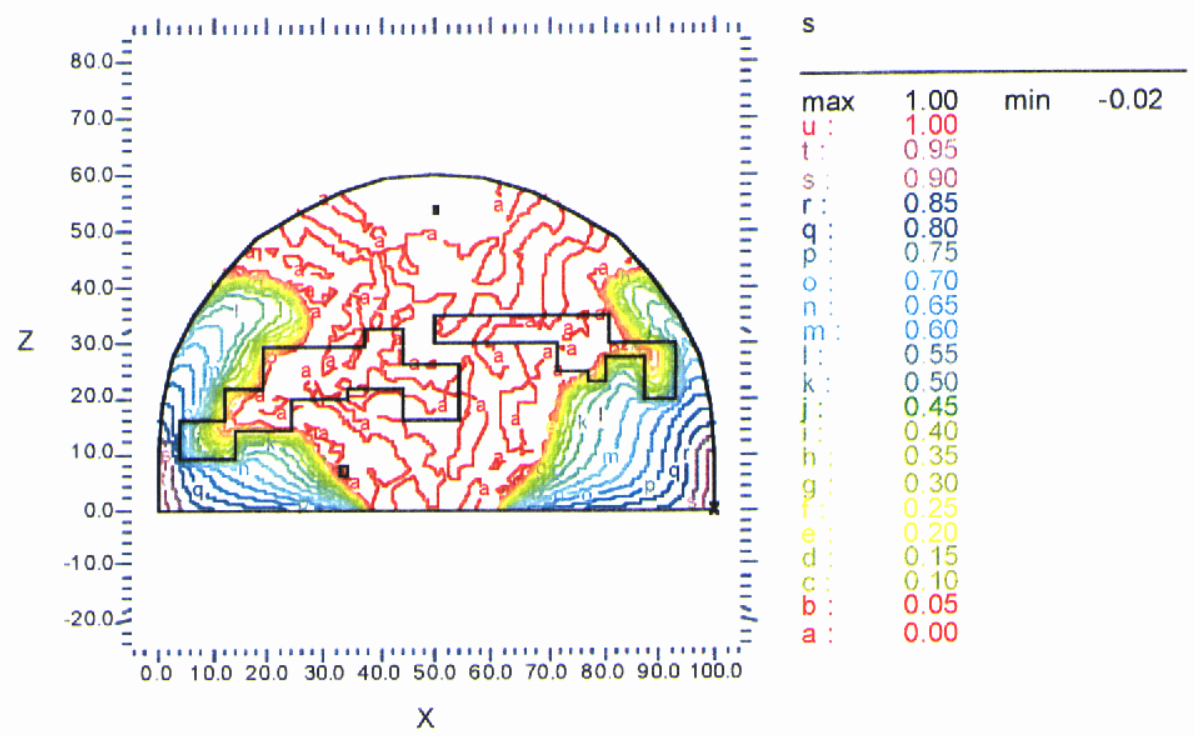

DMHSS1: Cycle $=121$ Time $=8.64 \mathrm{e}+6 \mathrm{dt}=2.1502 \mathrm{e}+5$ p2 5912 Nodes 2876 Cells RMS Err $=0.0016$ Integral $=1060.421$

Figure 9 - Isocontours of water saturation after 100 days with $R=1 / 10$

OW Water-Drive Heterogeneous sand/silt

$17: 30: 436 / 19 / 98$

FlexPDE 2.04

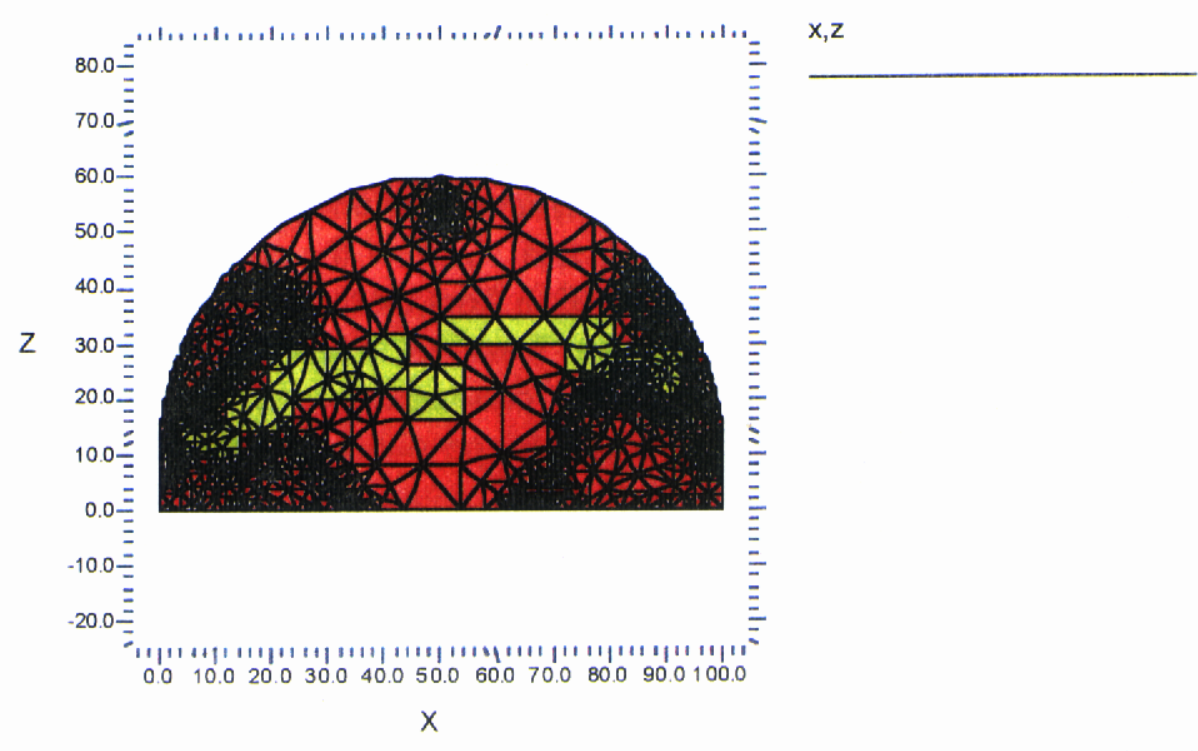

DMHSS1: Cycle $=121$ Time $=8.64 \mathrm{e}+6 \mathrm{dt}=2.1502 \mathrm{e}+5$ p2 5912 Nodes 2876 Cells RMSErr $=0.0016$

Figure 10 - Dynamically generated grid after 100 days 
OW Water-Drive Heterogeneous sand/silt

$17: 30: 436 / 19 / 98$

FlexPDE 2.04

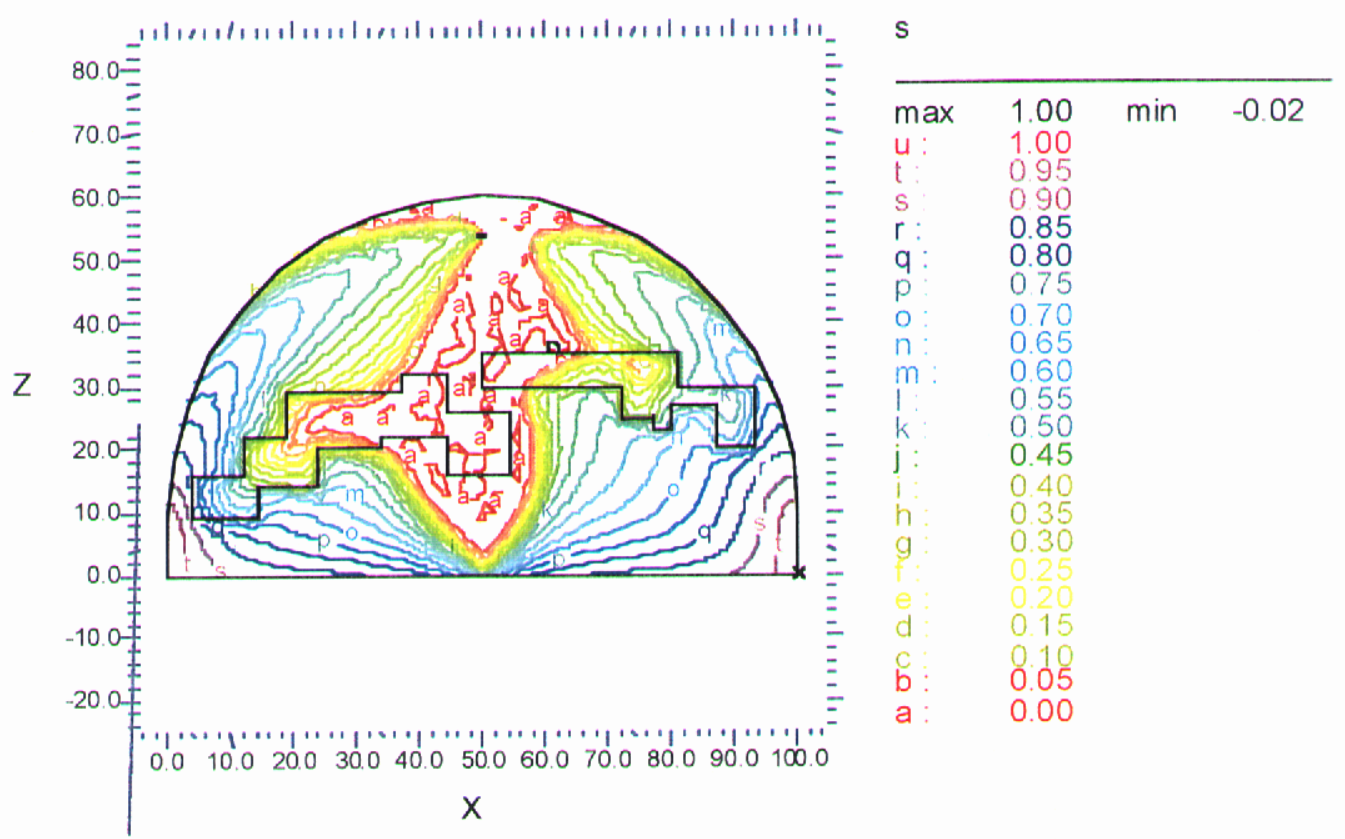

DMHSS1: Cycle $=171$ Time $=1.728 e+7 \mathrm{dt}=2.2799 \mathrm{e}+5$ p2 5936 Nodes 2902 Cells RMS Err $=0.0019$ Integral $=2140.259$

Figure 11 - First time arrival of water break-through after 200 days

OW Water-Drive Heterogeneous sand/silt

$17: 30: 436 / 19 / 98$

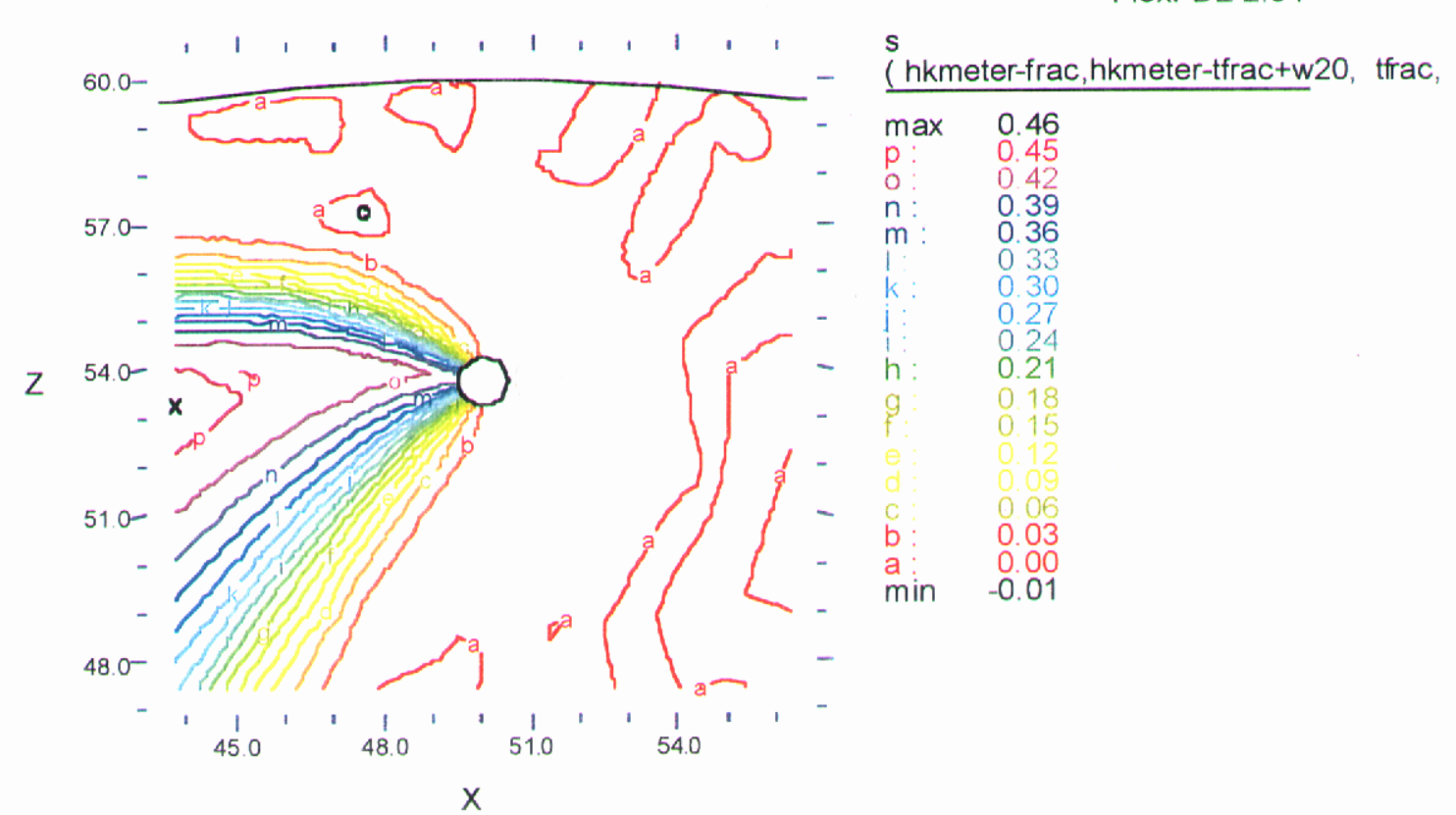

DMHSS1: $\quad$ ycle $=171$ Time $=1.728 \mathrm{e}+7 \mathrm{dt}=2.2799 \mathrm{e}+5 \mathrm{p} 25936$ Nodes 2902 Cells RMSErr $=0.0019$ Integral= 12.27777

Figure 12-Blow-up of first time arrival of water break-through at extraction well 


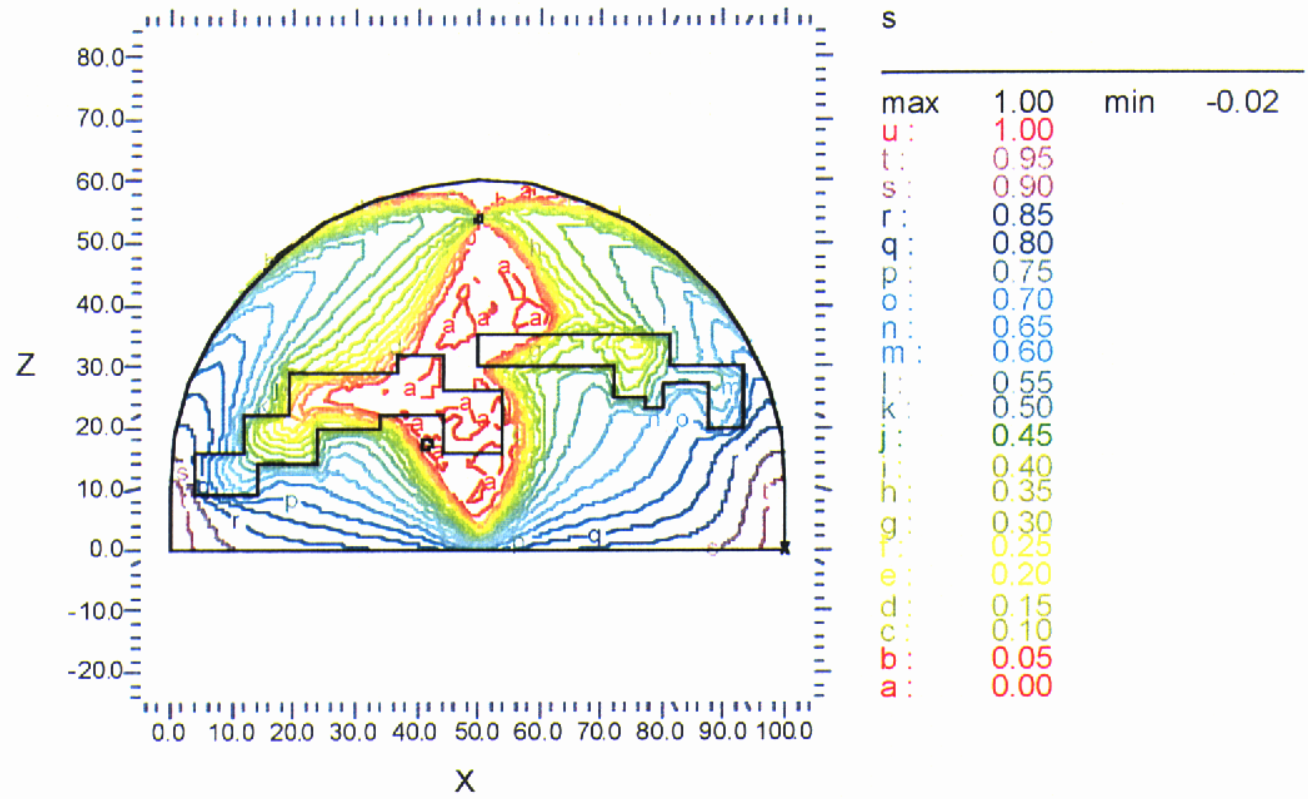

DMHSS1: Cycle $=187$ Time $=1.9008 \mathrm{e}+7 \mathrm{dt}=2.9579 \mathrm{e}+5$ p2 5954 Nodes 2912 Cells RMSErr $=0.001$ Integral= 2324.401

Figure 13 - Second water break-through at 220 days

OW Water-Drive Heterogeneous sand/silt

$17: 30: 436 / 19 / 98$

FlexPDE 2.04

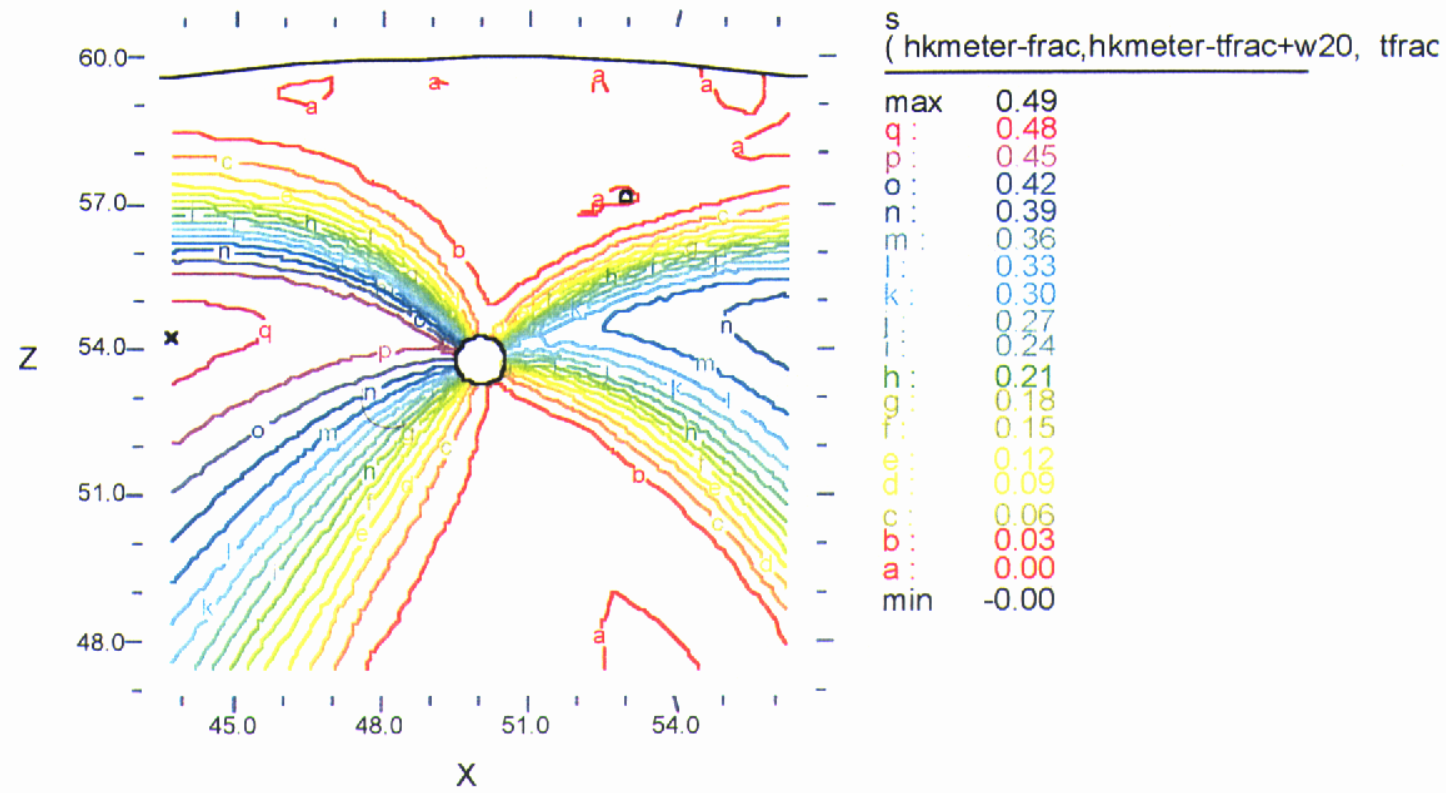

DMHSS1: Cycle $=187$ Time $=1.9008 \mathrm{e}+7 \mathrm{dt}=2.9579 \mathrm{e}+5$ p2 5954 Nodes 2912 Cells RMS Err $=0.00^{\circ}$ Integral= 23.30921

Figure 14-Blow-up of second water break-through at extraction well 


\section{Case 2: Forward solution for pressure and saturation with $R=0$}

The permeability in natural systems may vary by many orders of magnitude. In such instances the contrast ratio, $\mathrm{R}$, may approach zero in parts of the formation. The problem considered in this second case is identical to Case 1 above, except that $\mathrm{R}$ is here taken to be zero. The results shown in Figure 15 indicate that, at 100 days, the water saturation front has not penetrated the low-K substructures, which is in distinct contrast to the corresponding results in Case 1. Therefore retardation effects, in which water resides for some significant amount of time in the interchannel sediments before exiting through the downstream interface(s), are practically negligible in the present case. It is apparent in Figure 15 that saturation fronts simply go around the interchannel sub-structures; and the flow evolution pattern is somewhat different than when significant retardation occurs. Such behavior potentially affects oil production histories and thus remaining oil. Figure 16 shows that, after 420 days of pumping, oil is possibly trapped in several areas: (i) beneath the concavedownward regions of the low-K regions, (ii) in the narrow channel between the low-K regions, and (iii) in the vertical column area above the narrow channel. There is practically no retardation of oil in the low-K sediments at any time. After 2.3 years, approximately $70 \%$ of the oil has been produced; and it appears that most of the remaining 30\% is trapped, more or less indefinitely, beneath the low-K substructure (with the larger concavity) on the left-hand-side of the dome.

The results in these cases (as well as others that are not included in this report) suggest that correct handling of sharp saturation fronts and formation interfaces is important for simulating histories of oil recovery, retardation, and trapping. They further suggest that the shape, scale, and permeability contrast of formation heterogeneities may be significant determinants of critical transient flow processes in estimations of remaining oil.

OW Water-Drive Homogeneous Impermeable

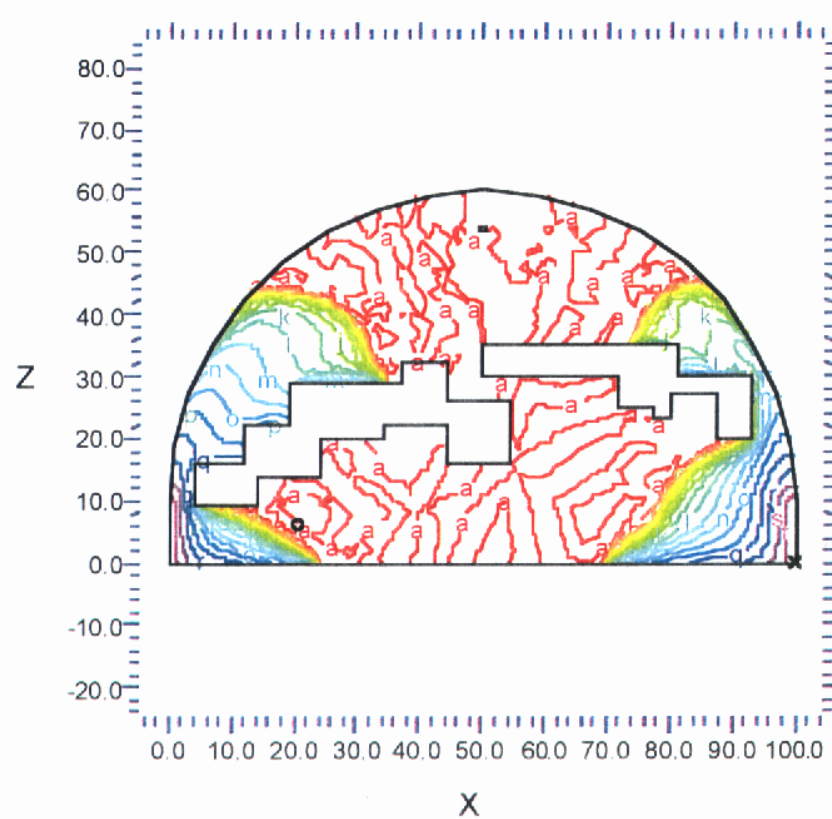

$19: 20: 056 / 25 / 98$

FlexPDE 2.04

s

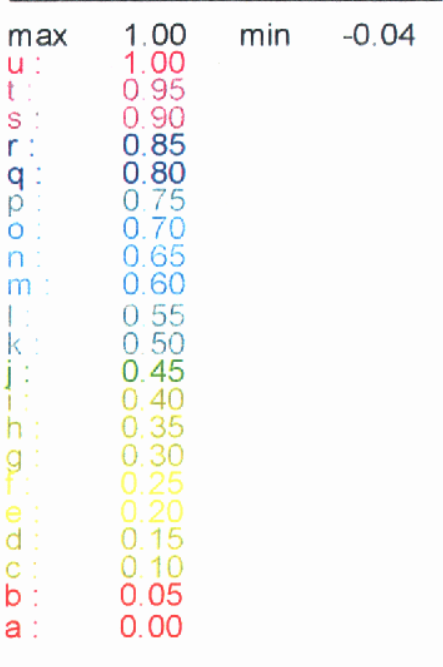

DMHI2: Cycle $=109$ Time $=8.64 e+6 \mathrm{dt}=2.071 \mathrm{e}+5$ p2 3710 Nodes 1728 Cells RMSErr $=0.0029$ Integral= 823.0667

Figure 15 - Isocontours of water saturation after 100 days for $\mathrm{R}=0$ 


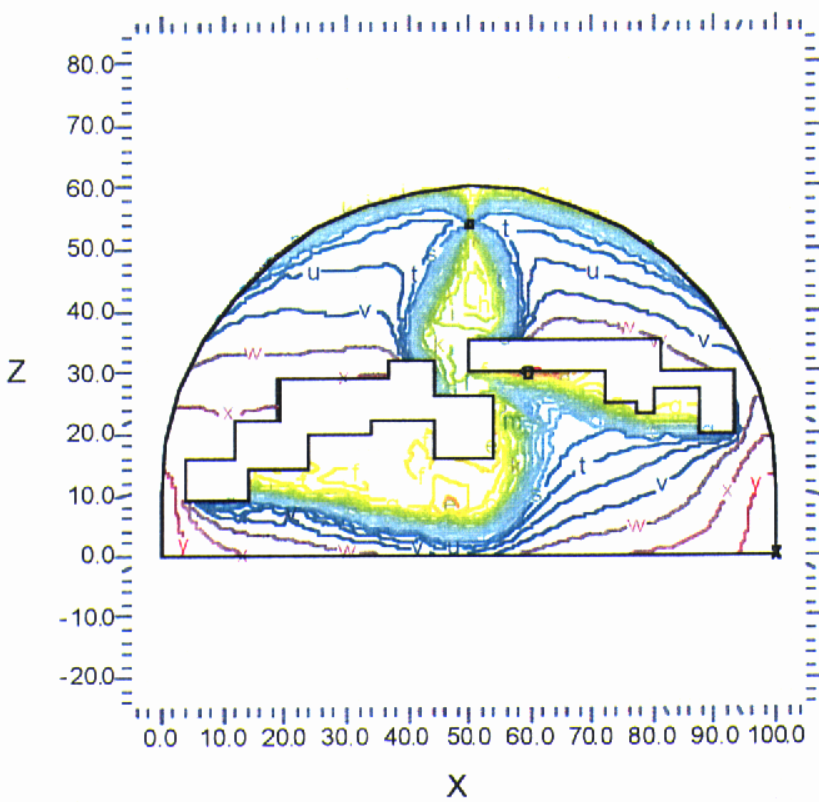

\begin{tabular}{llll}
\hline $\max$ & 1.00 & $\mathrm{e}$ & -0.05 \\
$\mathrm{z} \vdots$ & 1.00 & $\mathrm{~d}$ & -0.10 \\
$\mathrm{y} \vdots$ & 0.95 & $\mathrm{c}:$ & -0.15 \\
$\mathrm{x} \vdots$ & 0.90 & $\mathrm{~b}$ & -0.20 \\
$\mathrm{w}$ & 0.85 & $\mathrm{a}$ & -0.25 \\
$\mathrm{v}:$ & 0.80 & $\min$ & -0.26
\end{tabular}

DMHI2: Cycle $=183$ Time $=3.6288 \mathrm{e}+7 \mathrm{dt}=1.1208 \mathrm{e}+6$ p2 4073 Nodes 1897 Cells RMSErr= 0.0065 Integral $=2557.32$

Figure 16 - Isocontours of water saturation after 420 days for $\mathrm{R}=0$

\section{Inverse Model Verifications with Densely Sampled Data Sets}

\section{Case 3: Inverse solution for permeability (K) from dense pressure data}

The purpose of this example was to test the capacity of the inverse solution method, alone, for calculating a known heterogeneous permeability distribution from 'sufficiently dense' measurements of pressure at wellbores. As indicated previously, an inverse method that is expected to yield credible results for real situations with sparse data must first be able to produce high-fidelity images when given sufficiently dense data. The actual number of datum points that would constitute sufficiently dense sampling is generally nebulous, owing to both the problemspecific and nonlinear nature of reservoir parameter estimation problems. So benchmarking against ground-truth examples is imperative for gauging the potential effectiveness of any inverse technique. From signal-processing principles, pressure data are considered to be 'sufficiently dense' when simple interpolations (with F-I finite element nodes and basis functions) between datum points resolve the range of spatial frequencies that constitute good permeability images. High-quality images of permeability were obtained in the present case when many thousands to hundreds of thousands of pressure datum points were available. The results presented in Figures 17-19 were obtained with approximately 200,000 discretely sampled pressure datum points (Figure 20). The 'measured' pressure values were actually abstracted from ground-truth values at discrete wellbore locations; and spatial filtering PDEs were not included in this case.

Several observations can be made from results obtained in this case in Figures 17-19. First, considerable resolving power is attainable with the F-I techniques and the FlexPDE solution algorithms. The image quality obtained in Figure 19 is representative of many runs that were 
solved for this case, with some problem-specific differences that will be indicated later. Hence this case provides a useful henchmark for examining effects of data sparsity, which is always a central issue in practical reservoir applications. Finally, the image in Figure 19 demonstrates the fundamental fact that images from a finite number of datum points cannot be truly perfect (unique) - - no matter how densely they're spaced - - because some information is always lacking between the datum points. Although not a dramatic effect, as in sparsely sampled problems that appear later, spatial filtering was nominally beneficial in imaging densely sampled data. Problem-specific factors that affect images in these F-I solutions include: data density/sparsity, permeability contrast ratios at rock interfaces, information propagation modes, and robustness of the numerical algorithms.

Although spatial filtering PDEs were not incorporated in this case, the inverse solution process was nonetheless challenging for reasons that follow in a somewhat detailed discussion. We first note that, because fluid pressure equilibrates almost instantaneously, it satisfies the steady-state flow equations (E-22) and (E-19) at any given time(s). Consequently, the basic inverse technique (Appendix B and Gelinas et al [1998]) applied here solves the steady-state inverse equation (E-22) for the sum of all mobilities, $\mathrm{K} \bullet \mathrm{M}$ (Appendix E). Data that has been measured at some time shortly after pumping starts, $\mathrm{t}=0_{+}$, and before fluids have changed from their initial conditions, are used to solve for $K \cdot M$ (and $K$ ) in this test case. The discretely measured pressures at $t=0_{+}$are, in fact, the ground-truth values sampled at 200,000 datum points (Case 1). At $t=0_{+}$, fluid saturations are equal to their initial values, $S_{o}=1.0$ and $S=S_{w}=0$ everywhere in the dome; and the total mobility, $M$, is thus given by equation (E-18). The remaining information that is needed to solve the inverse equation are values of $\mathrm{K}$ on appropriate Cauchy lines (Appendix B). The vertical boundaries near the dome floor, where water enters/leaves the oil-dome, are taken to be Cauchy lines because all characteristics (streamlines) in the domain emanate from these boundaries and terminate at the extraction well. The 'measured' values of $\mathrm{K}$ on the Cauchy lines are $\mathrm{K}=\mathrm{K}_{\mathrm{C}}=5 \times 10^{-13} \mathrm{~m}^{2}$. (We note here that Cauchy lines can be selected in many different ways. This is an area that is under continuing study and promises to produce even better images from fewer datum points in future works.)

The above process of solving the inverse equation for $\mathrm{K}$ is a fair test of the techniques under development here, because the algorithms have no way of knowing that the 200,000 'measured' datum points are identically equal to the ground-truth values at discrete wellbore locations from Case 1. Figures $17-19$ show an evolving development of the K-distribution as its ultimate solution develops progressively from an arbitrary starting value (for $\mathrm{K}$ ) by an iterative process. In these solutions, equation (E-22) was regularized and solved for $\mathrm{K}$ in three stages. Progressively smaller values were assigned to the regularization coefficient, which maintains the integrity of the original hyperbolic PDE, in each stage. (Recall previous discussion that defined regularization of the hyperbolic saturation equation for the horizontal water-drive problem.) The regularized inverse equation in the present case is, however, much more difficult to solve than the PDEs considered in the previous water-drive problem (example problem 1). The linearized algebraic system in this case is so close to being singular (very large condition number) that it requires a much more robust solution technique than was used in previous examples. We therefore found it necessary to use (in FlexPDE ) a Vandenberg conjugate gradient algorithm with incomplete Cholesky pre-conditioning in order to solve for $\mathrm{K}$ in this case [Vandenberg, 1988; Jea and Young, 1983].

It is apparent in the captions of Figures $17-19$ that the number of adaptive-grid nodes tends to increase synchronously with the quality of the K-images, until the demanded accuracy is achieved 
in each stage of the solutions. From a signal-processing point of view, increasing the number of spatial grid nodes is tantamount to incorporating higher and higher spatial frequency components, which enhance the spatial resolution of the K-images in each stage of development. Viewed as an input-output system, the output solutions from FlexPDE are K-images composed of spatial frequency components that were 'signalled' by the input pressure (Figure 20) and the initial Cauchy data acting through the inverse flow equation. The flow equation acts essentially as a nonlinear transfer function. The quality of the image depends upon the capacity of available grid nodes to resolve the spatial frequency components of the $\mathrm{K}$-image consistently with the sampling density. Clearly, a failure to solve the inverse flow equation with high-accuracy would add distortion to the output image of $\mathrm{K}$, which could not be distinguished from noise (or errors) in the input pressure data. FlexPDE, like many other robust PDE solvers, achieves high levels of accuracy by using both local and global error diagnostics to dynamically control the location, nature, and extent of adaptive grid refinements and un-refinements. The utility of unstructured adaptive-grid PDE solvers in this work stems largely from their capacity to place grid nodes where they are most needed, without fixed topological structure constraints, and when they are most needed for resolving physical features that are commensurate with the information contained in the discretely sampled pressure and permeability data.

Returning to the results in Figure 19, the permeability contrast ratio was equal to $\mathrm{R}=1 / 10$; and the K-isocontours conform generally to the shape of heterogeneity substructures in the oil-dome. But, even with extremely dense datum points the image of the low- and high-permeability interfaces in Figure 19 is not completely sharp. In addition to the absence of information between datum points, image resolution can be affected by such factors as numerical error (from PDE discretization schemes, matrix condition, and regularization), data noise, derived pressure gradient error, and possibly effects associated with mathematical instability. Notice that errors from noisy Laplacians of pressure in many other inverse techniques do not occur here, because finite element methods avert Laplacian evaluations via an integration-by-parts of divergence terms in flow equations. While results to-date are certainly promising, we have gained deepened insights during Phase I that will potentially yield still greater levels of image resolution from less data in future efforts. 
OW Water-Drive Homogeneous Impermeable

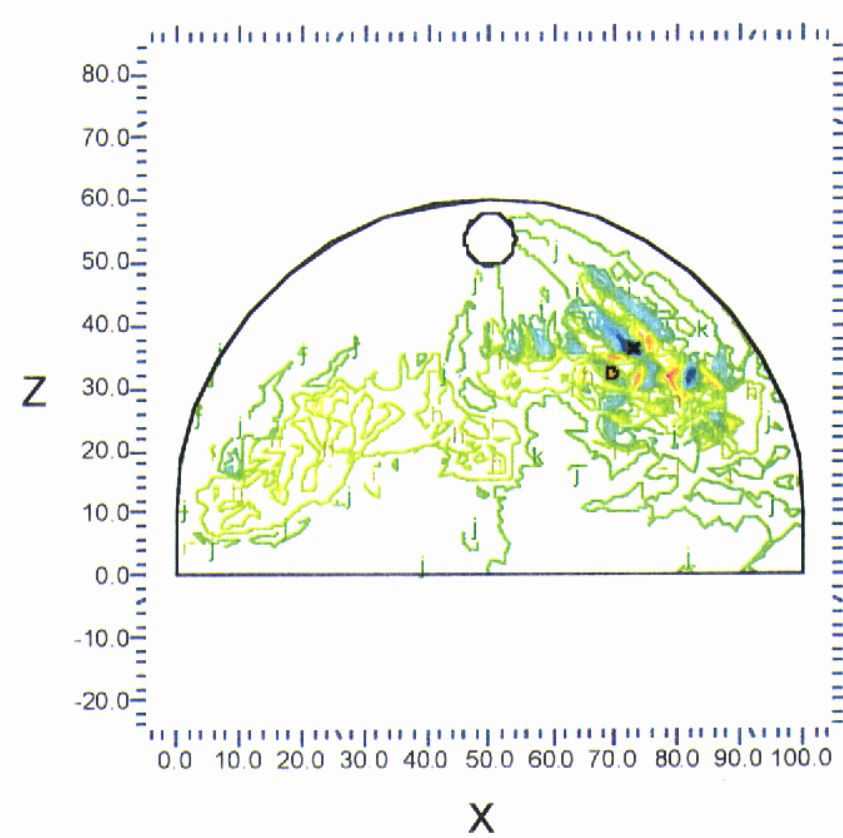

14: $18: 40$ 6/27/98

FlexPDE 2.04

DMINV4: Grid\#2 p2 834 Nodes 388 Cells RMS Err $=0.0447$ Integral $=3783.017$

Figure 17. Recovery of K from densely sampled pressure data; 834 nodes; Stage 1.

OW Water-Drive Homogeneous Impermeable

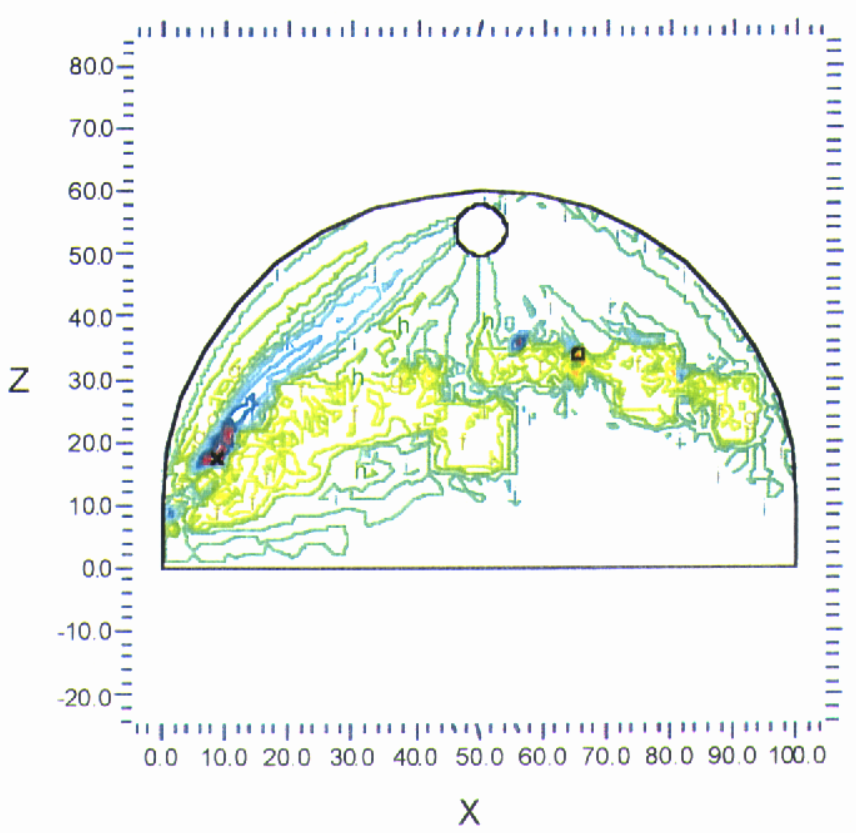

$14: 18: 406 / 27 / 98$

FlexPDE 2.04

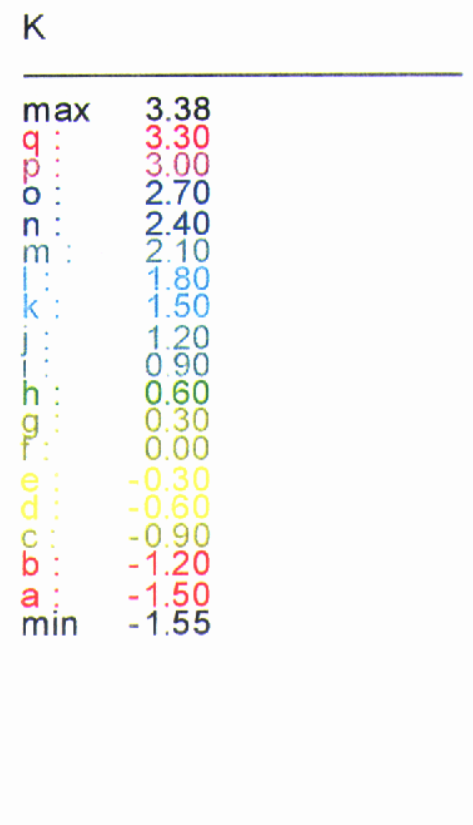

DMINV4: Grid\#1 p2 1717 Nodes 817 Cells RMS Err= 0.0239 Integral= 3917.034

Figure 18. Recovery of K from densely sampled pressure data; 1,717 nodes; Stage 2 . 
OW Water-Drive Homogeneous Impermeable

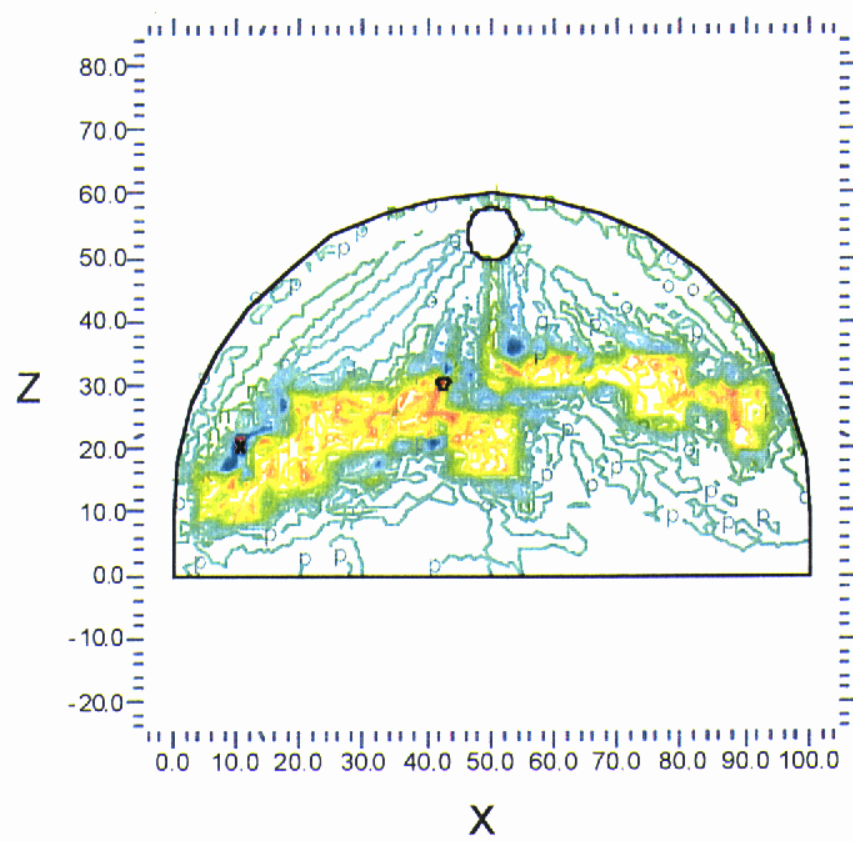

14:18:40 6/27/98

FlexPDE 2.04

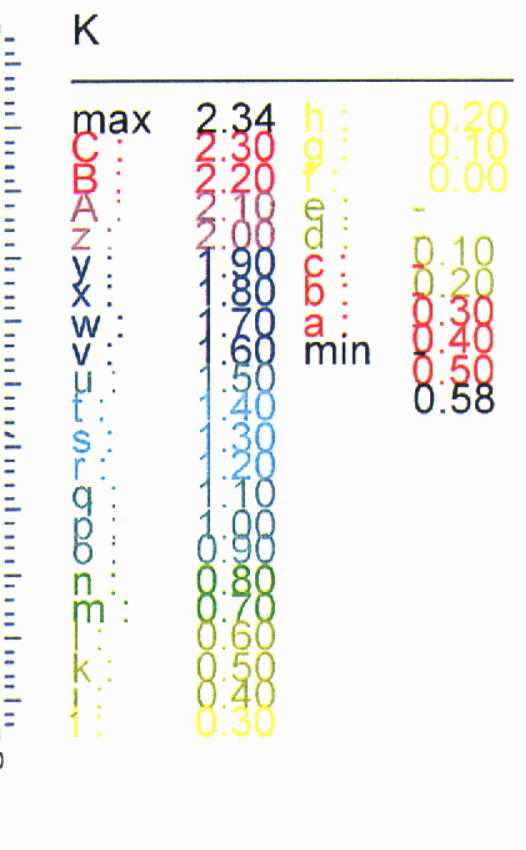

DMINV4: Grid\#1 p2 2471 Nodes 1175 Cells RMS Err= 0.0326

Integral $=4048.382$

Figure 19. Recovery of K from densely sampled pressure data; 2471 nodes; stage 3. 


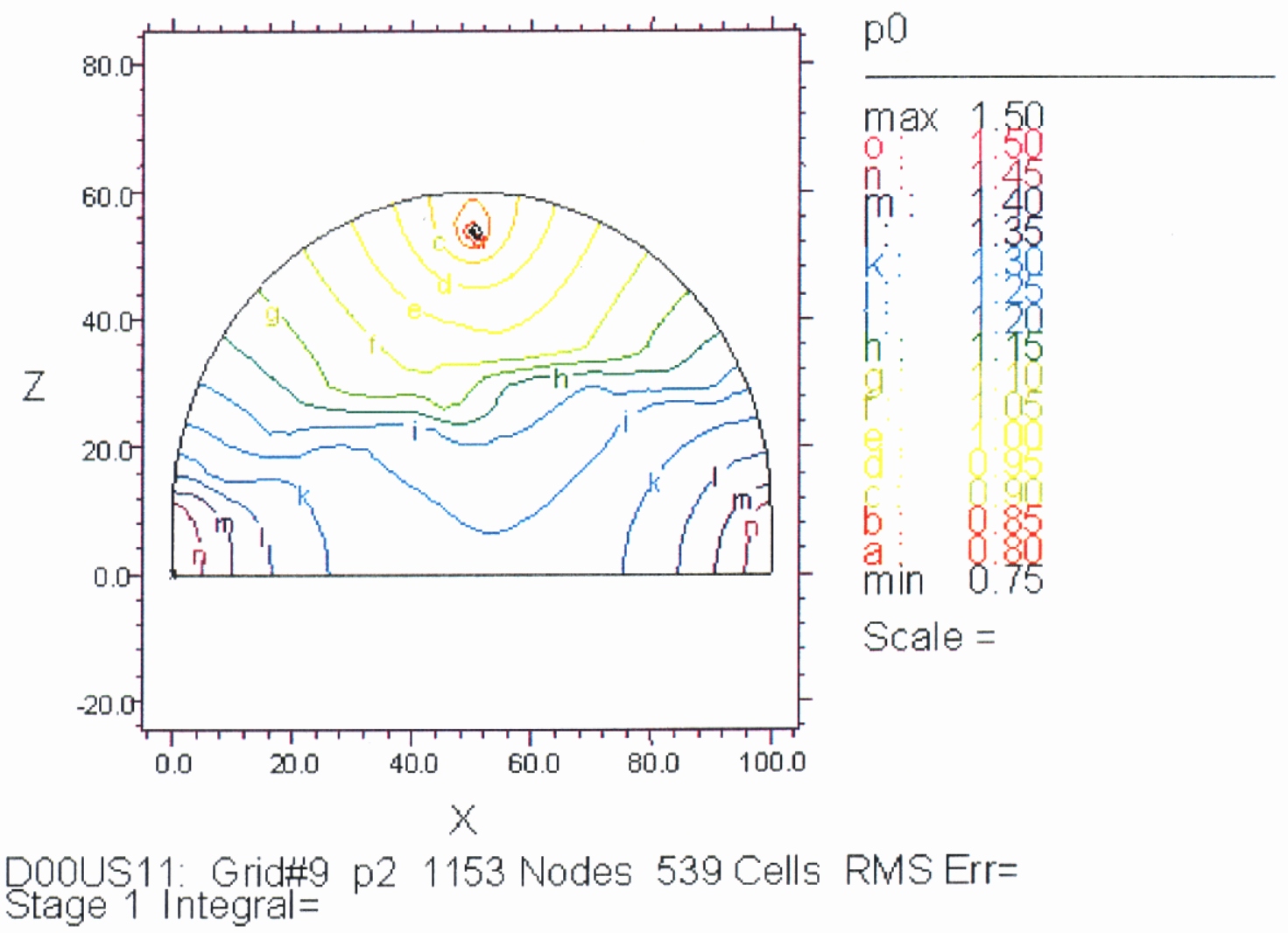

Figure 20. Isocontours of densely sampled pressure data $(t=0+$ snapshot $)$

\section{Inverse Model Verifications with Sparsely Sampled Data Sets}

The purpose of the remaining cases in this report was to test the capacity of the F-I techniques for producing credible images of heterogeneous reservoir formations from sparsely located pressure 'measurements'. As was true in previous cases, the number of datum points that will be needed to produce credible images from sparse data is nebulous. Also, the potential role and effectiveness of spatial filtering has not previously been established in this type of inverse method, to the best of our knowledge. We therefore benchmark representative cases against ground-truth examples to measure the potential effectiveness of this inverse technique, having previously noted the method-specific, problem-specific, and nonlinear nature of reservoir parameter estimation. When dealing with sparse pressure data, the inverse solution technique described in Case 3 is augmented with additional PDEs that spatially filter the derived pressure gradients. Spatial filtering is necessary because interpolations that respect both the flow equation and the discrete data are noisy. Recall that pressure data were interpolated between wellbores by finite element solutions of the flow equation, which were "clamped" to measured values at discrete wellbores. Unless conditioned by spatial filtering, the interpolations would not know that they must be "bandlimited" functions prescribed by undersampling theory [Bracewell, 1986]. Expressed in terms of 
Fourier transforms, undersampling of signals by discrete measurements produces images with the high-frequency tail truncated beyond some spectral cutoff. Values of the cutoff frequency are related to the spatial intervals between measurements. The truncated high-frequency information is known to masquerade at lower frequencies in the cutoff spectrum; and this effect is referred to as "aliasing". If the high-frequency tail is not too important, sparse sampling procedures produce fair images. If allowed to persist, high-frequency interpolation noise is magnified in derived pressure gradients that are then used to solve the inverse flow PDE (equation (E-19)) for permeability. The basic technique for solving spatial-filtering PDEs simultaneously with an inverse flow PDE is described in Appendix B for ground water flows. See equations (B-17a) - (B-17d). These techniques, with suitable modifications for dealing with transient snapshots of pressure and other data, have been extended successfully to reservoir problems in Phase I.

\section{Case 4: Sparsely Sampled Cases - Spatial Filtering Applied}

Case 4 is actually made up of four individual problems in which a permeability image is calculated from four sparsely sampled data sets composed of 1,20,75 and 300 pressure datum points, respectively. Each data set was abstracted at discrete measurement locations at time $=0_{+}$, as in Case 3. The full F-I method was applied to each of the data sets. The resulting permeability images are presented in Figures 21-24, proceeding from the least-sparse (300 datum points) to the most-sparse ( 1 datum point) data sets.

The F-I solutions were stable; and the images were plausible relative to ground-truth in all cases when due account is taken of data sparsity. Figures 21 and 22 indicate that very credible images of formation heterogeneity were obtained commensurate with the 300 and 75 datum points that were given. The image obtained from 20 datum points in Figure 23 gives a plausible realization of low permeability horizontally across the mid-region of the domain. The image in Figure 24 was calculated from a single pressure datum point 'measured' in the middle of the domain. This case confirms that the F-I method was stable in the most extreme conceivable circumstance, with one pressure measurement. There was, of course, other information supplied to the problem via the 'known' permeability and hydrostatic pressure values on Cauchy lines at reservoir-aquifer interfaces, by 'measured' pressure conditions at the production well, and by the no-flow boundary conditions on the remaining boundaries of the oil-dome. The results in these cases are generally representative of many other runs that were executed with widely varying numbers of 'measured' datum points. In many cases with relatively sparse data ( $5-20$ datum points) image resolution sometimes exceeded our initial expectations from simple data-sampling/signal-processing principles. Image resolution improved progressively as the number of datum points increased, as expected. Finally, experience and insights gained in this initial phase of work suggested several ways to possibly improve the quality of these images with fewer datum points in future work. 


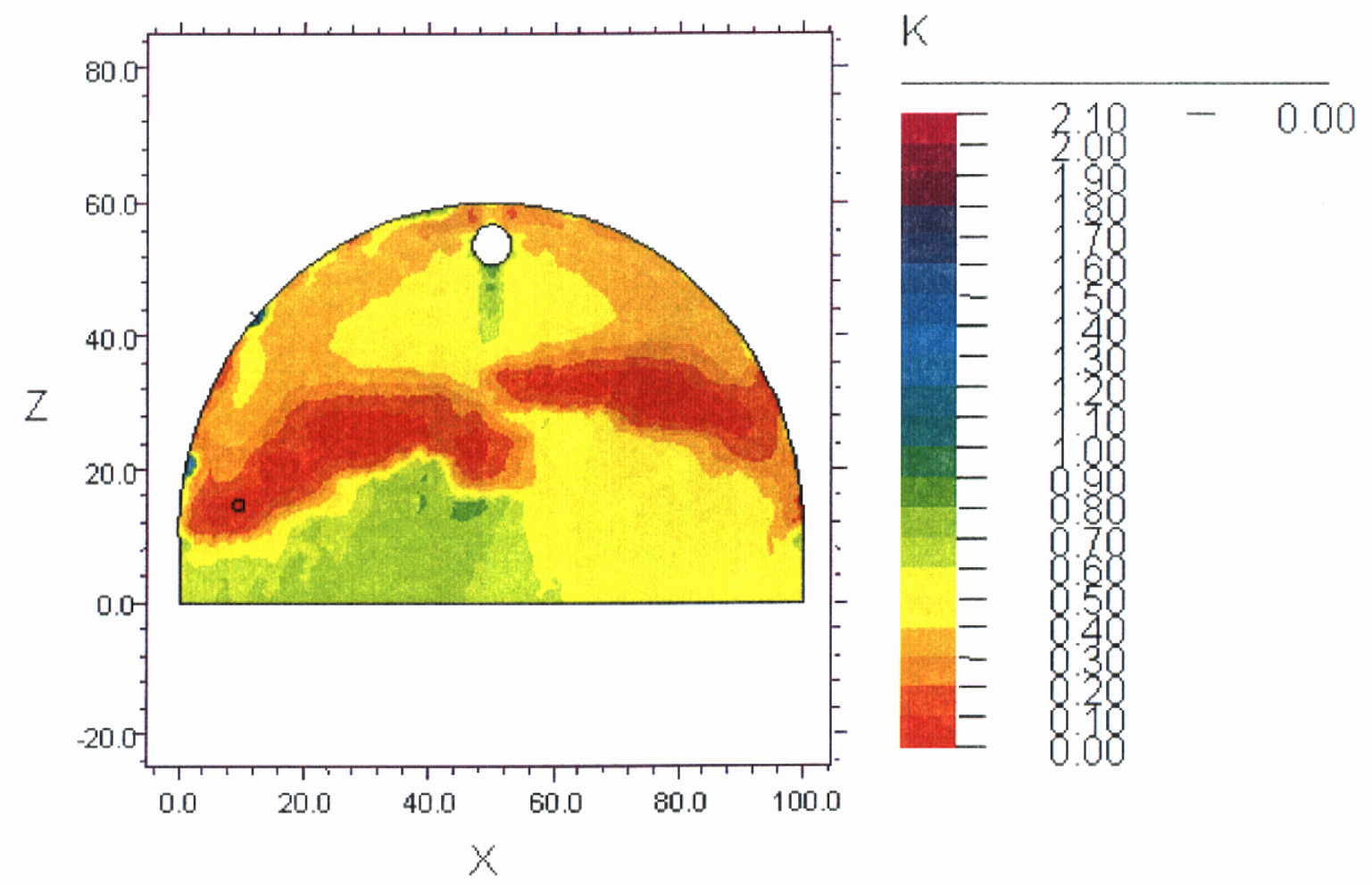

D20: Grid\#9 p2 12211 Nodes 5983 Cells RMS Err= Stage 1 Integral=

Figure 21. Calculated permeability image from approximately 300 pressure datum points, with spatial filtering applied. 


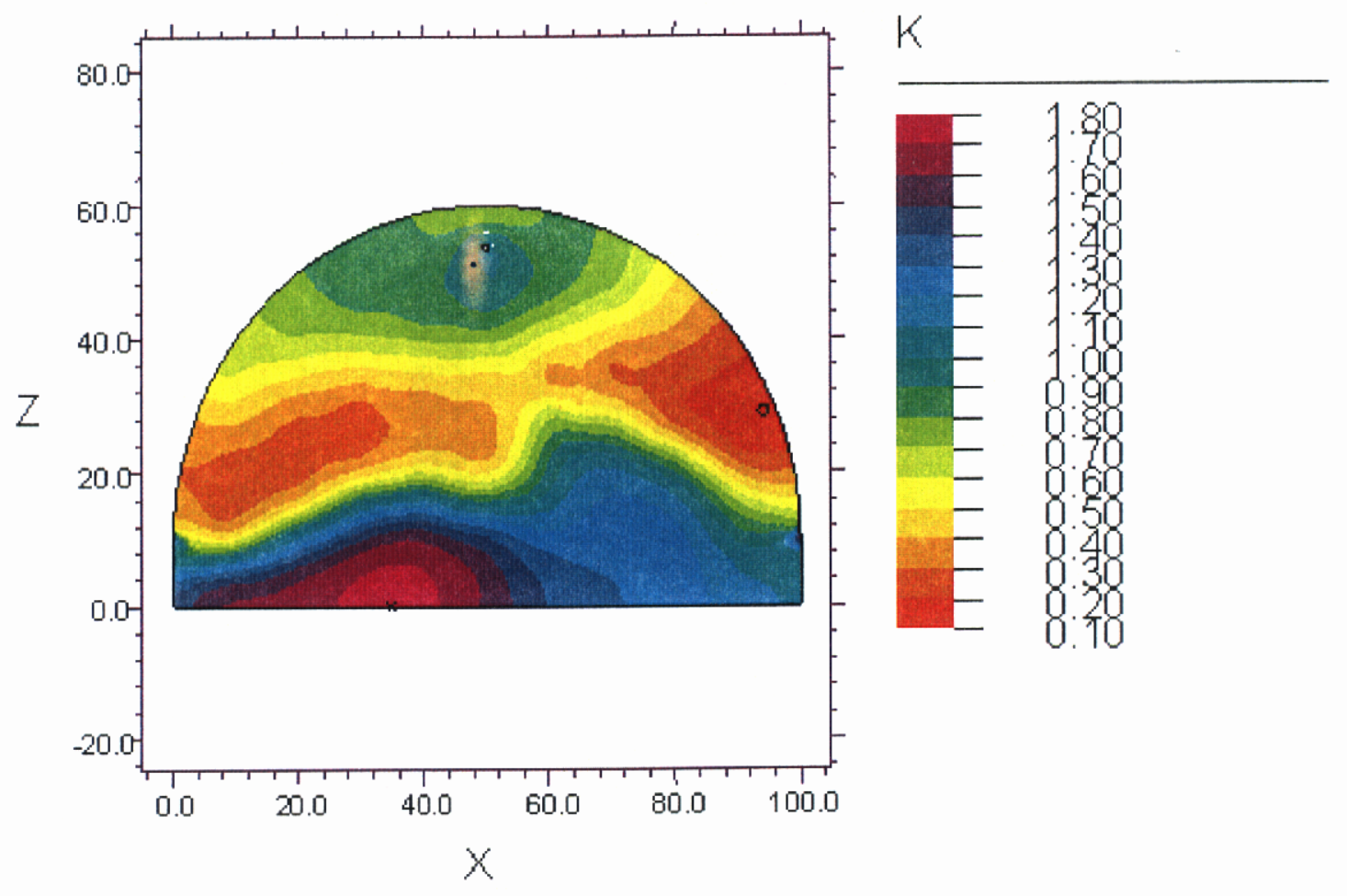

D81US11: Grid\#9 p2 2179 Nodes 1043 Cells RMS Err=
Stage 2 Integral=

Figure 22. Calculated permeability image from approximately 75 pressure datum points, with spatial filtering applied. 


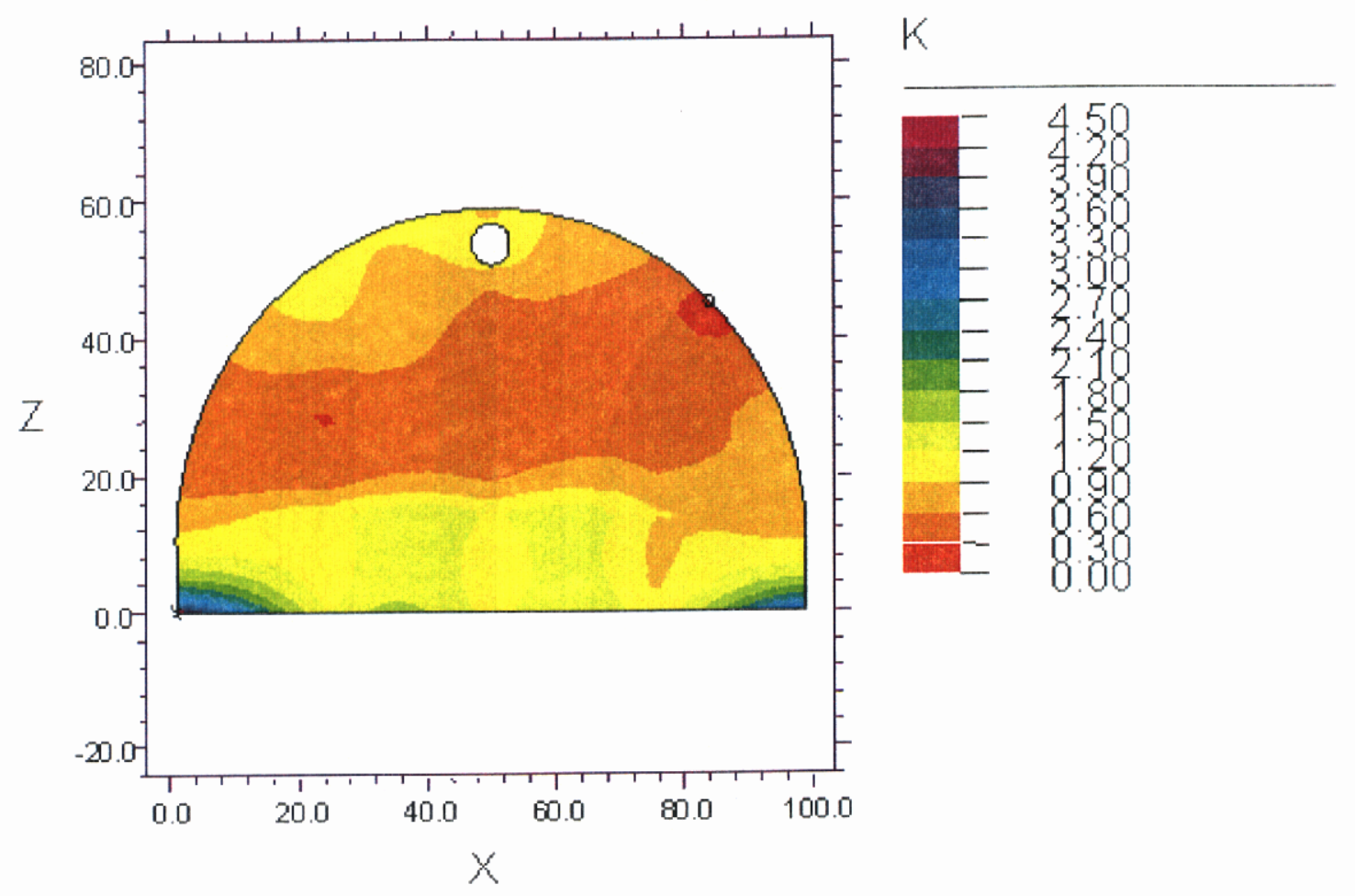

D5: Grid\#7 p2 1404 Nodes 664 Cells RMS Err= Stage 1 Integral=

Figure 23. Calculated permeability image from approximately 20 pressure datum points, with spatial filtering applied. 


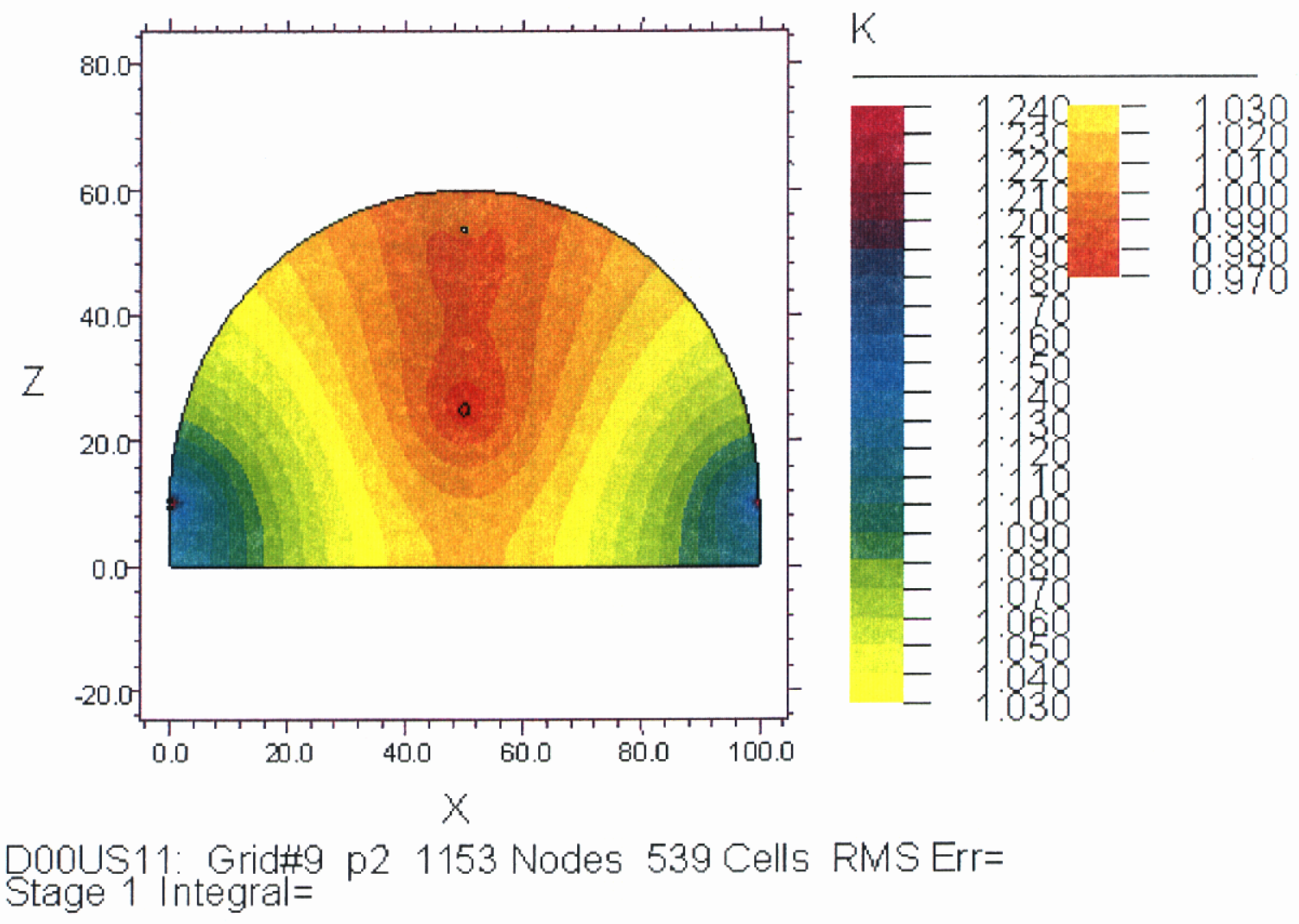

Figure 24. Calculated permeability image from one pressure datum point, with spatial filtering applied.

\section{Case 5: Sparsely Sampled Cases - No Spatial Filtering Applied}

The same data sets that were tested with the full F-I methodology above (Figures 21-24) were solved here with spatial filtering eliminated. The objective was to examine the potential benefits that spatial filtering can provide. The results were immediate and compelling. Permeability solutions were unstable and fraught with artifacts when pressure data was too sparse. Specifically, the unfiltered solutions in the oil-dome example were unstable when fewer than approximately 100 'measured' pressure datum points were used. Figure 25 presents the image that was obtained without spatial filtering in the case with 75 pressure datum points. We also observed that images obtained with no spatial filtering and 300 pressure datum points did not approach the quality of the images shown in Figure 22 with spatial filtering applied for 75 datum points. 


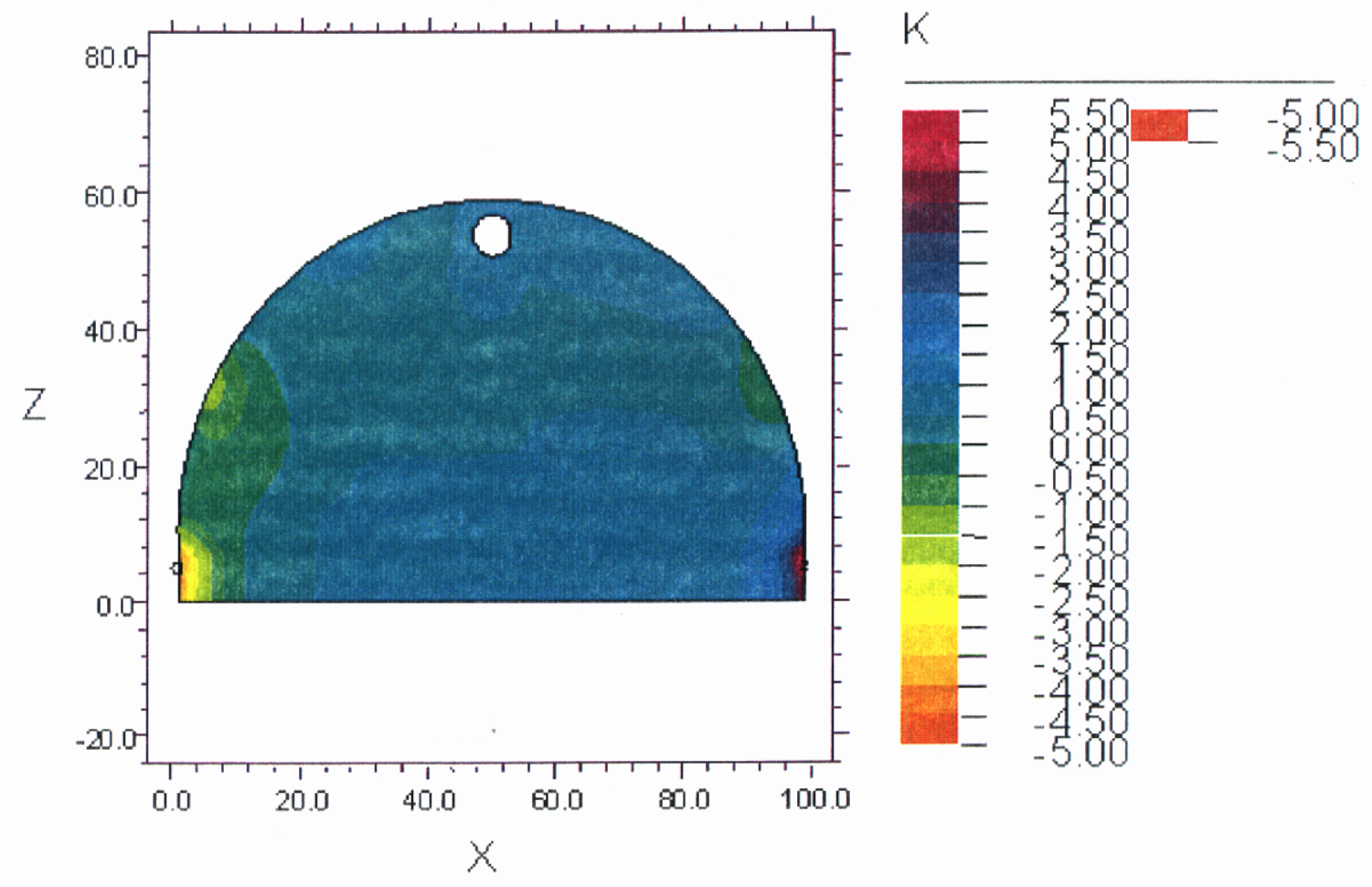

Q10: Grid\#10 p2 6535 Nodes 3195 Cells RMS Err= Stage 1 Integral =

Figure 25. Permeability image calculated from approximately 75 pressure datum points. With no spatial filtering applied, this inverse solution was unstable.

\section{IV-2 Synopsis of Examples and Results}

Owing to the breadth of test cases, results, and diverse motivations in this section, we present a concluding synopsis for interested readers.

\section{Example Problem 1:}

The first example was an idealized water-drive scenario on a 2-D horizontal domain (Figure 1). The absolute permeability was assumed to be spatially constant, and gravitational forces were neglected. Specific objectives in this example problem were to benchmark: (i) conceptual model formulations, (ii) FlexPDE solution algorithms, and (iii) forward solutions for fluid pressure and saturations. The results obtained with FlexPDE were found to compare favorably with results presented by Saad and Zhang [1998], who used an alternative adaptive-grid solution technique. Our results (Figures 2-7) indicated that the evolution of sharp fluid fronts and flows near extraction wells (with robust outflow boundary conditions) are resolved very well relative to most other numerical solution techniques. These are significant factors for determining the times, paths, and rates of fluids arriving at production wells, as well as the distribution of oil remaining in the formation over time. 


\section{Example Problem 2:}

The second example was an idealized vertical 2-D cross-section of an oil-dome. It loosely incorporates Pampano-like field data as depicted in Figure 8. Rock hetereogeneity was modeled with two absolute permeabilities; $\mathrm{K}_{\mathrm{C}}$ represented channel sands, and $\mathrm{K}_{\mathrm{I}}$ represented interchannel permeability. The contrast ratio at heterogeneous formation interfaces is given by $R=K_{I} / K_{C}$. An extraction well with an assumed down-hole pressure value (maintained by pumping) was located near the dome crown. The governing PDEs included gravitational effects (Appendix D). Pressure assignments at bounding aquifer interfaces were assumed to be time-invariant; and dome boundaries were assumed to be impermeable, except at the supporting aquifer interfaces.

A primary objective in this example was to benchmark inverse solutions for permeability. Test cases were solved for absolute permeability distributions from discretely 'measured' pressure datum points. The 'measured' datum points were, in fact, abstracted from ground-truth forward model solutions at designated wellbore co-ordinates. The density of datum points used to estimate permeability spanned a broad spectrum, ranging from extremely dense sampling $(>200,000$ datum points) to extremcly sparse sampling (one datum point). Key issucs in thesc cases were: "What is the best image quality that might be expected from F-I permeability interpolations between wellbore measurements for data sampling densities that range from very sparse to very dense?"; "How to extract the greatest amount of information from sparse data sets for reservoir systems, noting that such systems do not generally satisfy linearity and other assumptions that are needed for conventional Fourier-analysis-based signal-processing techniques?"; "How to implement spatial filtering techniques in physical configuration space, as an alternative to Fourier-transform techniques?"; and "How to suppress noise in both forward and inverse model simulations?" These issues are critical to successful advances toward project end-objectives. The results obtained to-date are encouraging.

\section{Forward Model Verifications (Cases 1 and 2):}

To address the issues above (and others), we first verified that highly accurate forward solutions were attainable for pressure and saturation in the oil-dome example. A first test case assumed that heterogeneous formation interfaces had contrast ratio, $\mathrm{R}=1 / 10$; and the second case assumed that $\mathrm{R}=0$. Results in Cases 1 and 2 (Figures 9-16) confirmed that FlexPDE algorithms resolve the propagation of disparate fluid pressure and saturation fronts extremely well for even the sharpest interfaces $(\mathrm{R}=0)$ in heterogeneous formations. FlexPDE solutions clearly tracked the complex evolution of saturation fronts, which could split, refract, and undergo retardation as they propagated through the reservoir. Depending on values of $\mathrm{R}$, the relative roles of individual physical processes, e.g., entrapment, refraction, and retardation were problem-specific and could affect the transient evolution of recovered and remaining oil over time.

\section{Inverse Model Verifications (Cases 3-5):}

The remaining cases in this section solved inverse problems for permeability distributions from data sets with varying degrees of sparsity. Because the resolution of permeability images is generally an unknown function of data density, we tested the importance of spatial filtering in F-I solutions versus sampling density. Densely sampled data sets were benchmarked in Case 3, and sparsely sampled data sets were considered in Cases 4 and 5. 


\section{Densely Sampled Data (Case 3):}

Case 3 tested the capacity of the inverse solution method, alone, to calculate a known heterogeneous permeability distribution from densely sampled data. The working principle is: If an inverse method is unable to produce a high-fidelity image when given sufficient data, there is little reason to expect the method to yield credible results for real situations with sparse data. Results in Case 3 (Figures 17-19) yielded high-quality images of permeability relative to ground-truth when sufficiently dense data was given (thousands to hundreds of thousands of datum points, depending on problem specifics). These results demonstrated that considerable resolving power is attainable with the F-I techniques and the FlexPDE solution algorithms. They further provided a useful basis for benchmarking the effects of data sparsity, which is always a central issue in practical reservoir applications. Finally, they supported the fundamental fact that images from a finite number of datum points cannot be truly perfect (unique) - - no matter how densely they're spaced - - owing to the absence of information between datum points. Spatial filtering was generally beneficial to image quality in densely sampled cases, but not to the dramatic extent that is observed with sparse data sets. Problem-specific factors that affected the impact of spatial filtering in F-I solutions include: data density/sparsity, permeability contrast ratios at interfaces, information propagation modes, and robustness of the numerical algorithms.

Sparsely Sampled Data - With Spatial Filtering (Case 4):

Case 4 solved for permeability from sparsely sampled data sets, which contained 1, 20, 75, and 300 pressure datum points, respectively. The full F-I method was applied, in which spatial filtering PDEs were solved simultaneously with the forward and inverse flow equations. The solutions were stable and the images were plausible relative to ground-truth, commensurate with the amount of available data, in all cases. The results obtained in these cases were generally representative of numerous additional runs that were executed with varying numbers of 'measured' datum points. In many cases with relatively sparse data (5-20 datum points) image resolution sometimes exceeded our initial expectations from elementary data-sampling/signal-processing principles. Image resolution improved progressively as the number of datum points increased, as expected.

Sparsely Sampled Data - No Spatial Filtering (Case 5):

Case 5 tested the potential benefit of spatial filtering by deleting it altogether. The results were immediate and compelling. Permeability solutions were unstable and fraught with artifacts when pressure data was too sparse -- as is true in many inverse techniques. Specifically, unfiltered solutions in the oil-dome example were unstable when fewer than 75 'measured' pressure datum points were used. This was a dramatic departure from the stable images that were consistently obtained with spatial filtering applied.

\section{Concluding Remarks:}

It is apparent in these Phase I results that the combined signal-processing and physics-based FI techniques, solved wilh rigorous mathematical and numerical methods, enhance the resolution of images of permeability away from wellbores. In some instances, where many other existing techniques would be unstable, the F-I method was stable and produced surprisingly good images from sparse data. Insights gained in Phase I suggest that additional advances may materialize as these incipient principles and solution techniques are developed more fully over time. For example, results and descriptions in Section IV indicate that the evolution of sharp fluid fronts, which may 
split, refract, and undergo retardation as they propagate through the reservoir, are resolved accurately in forward BOM solutions with these modeling techniques. Data sets observed at specified times (or snapshots) during transient wellfield operations may serve as additional constraints for enhancing permeability images. The results further suggest that oil recovery is a function of low- and high-permeability contrasts, of physical scale and shape of rock heterogeneity, and of disparate-scale flow and saturation gradient evolution. A capacity to better resolve these sensitive features with multiple data snapshots and enhanced images of reservoir processes away from wellbores potentially opens promising new paths toward attainment of far-market objectives.

\section{Recommendations}

From a critical evaluation of Phase I results we believe that continuation of this project to a Phase II effort is warranted and would recommend it to those who understand the risk-versuspotential return factors in far-market endeavors. A primary objective in Phase II would be to further expand the concepts and methods proven in Phase I to more realistic 2-D porous media physics. This would include: (i) representative capillary pressure curves and other relevant reservoir data, (ii) investigating the effects of undersampling in reservoir contexts, and (iii) incorporating timedependent production data with other transient data. We believe that it is important at this stage of development to rule out possible show-stoppers in three essential areas before expending the much greater levels of effort that would be required with 3-D codes and additional technical scope in a final Phase III: First, the extension of capillary physics extends the range and possible severity of nonlinear coupling and uncertainty effects that need to be assessed. Second, the nature and significance of image distortion between boreholes must be assessed for a sizeable range of small and large data sets. These results would provide an early view of the value of individual pressure measurements and the significance of their number and locations relative to production and/or injection wells. Third, the inversion techniques applied for steady-state ground water problems (Appendix B) will extend, theoretically, to inversions of data gathered at multiple times during wellfield operations. This is a key concept for economically enhancing image resolution from sparse data, which needs to be confirmed in 2-D, with oil-water physics. Each of these are formidable tasks that we believe can, and must, be resolved in Phase II, on the path toward the final far-market goals. 


\section{Appendix A: Theory}

Although physical systems may sometimes behave deterministically, they are nonetheless stochastic in their basic nature because fundamental uncertainties exist at all scales of measurement. Consequently, both dynamics and statistics must be considered in any modeling effort that seeks to gain a fuller understanding of the information contained in measured data and then use it to produce more reliable predictions of subsurface properties and flow behavior. The theoretical basis of stochastic modeling approaches is found in kinetic theory, where the dynamical and statistical axioms of physics are used to generate continuum flow models of mass, momentum, and energy balance. Two theoretically equivalent approaches are found. One derives from the work of Langevin [1908] and is the basis of many of the random field models that are used extensively in subsurface flow problems today. Random field techniques focus attention on evaluations of fluctuations of both dynamic variables and constitutive property distributions about their respective means. At their lowest statistical order, fluctuations are characterized by (second-order) co-variances and cross-covariances. They are evaluated by numerous techniques that include: inference of prior information, stipulated properties, estimators from functional analysis, perturbation analyses, fluctuation PDEs, maximum likelihood, etc. (See for example, McGlaughlin and Townley [1996]; Gelhar [1993]; Dagan [1989]; Harter, Gutjahr, and Yeh [1996]; Hsu, Zhang, and Neuman [1996]; among many others.) The other, less-used, approach is based on a hierarchy of partial differential equations (PDEs) for statistically averaged moments of constitutive properties and flow variables [Gelinas, Doss, Ziagos and Nelson, 1998, Neuman and Orr, 1993, and Dagan, 1989]. Moment PDE heirarchies can be derived using either Langevin techniques or axiomatic techniques of classical and/or quantum physics, as discussed further below. When attempting to determine formation properties from measured data, both random field and moment PDE approaches seek to replace an ill-posed inverse problem by a well-posed one. Distinctly different formulations and solution techniques are used to represent and solve the defining equations in the respective approaches. In addition to subsurface flow problems, the random field and moment PDE hierarchy approaches have been applied for several decades in numerous other technical disciplines, for both classical and quantum systems. (See, for example, Osborn [1963]; Gelinas and Osborn [1966], [1967]; Osborn et al [1967], [1968], Akcasu and Osborn [1966], Lax [1966], Gelinas [1976], among many others.) Advances in numerical PDE solution techniques and software tools have recently helped to resolve some of the longstanding issues that previously hampered application of the moment PDE approach in ground water problems [Gelinas et al, 1998]. With the reasonable prospect of extending the new ground water techniques successfully to reservoir problems, the moment PDE approach was applied in this Phase I DeepLook project. A more specific description of Langevin and moment PDE approaches is sketched immediately below in the context of reservoir problems.

\section{Langevin techniques for random fields}

With the possible exception of manual history-matching, variants of basic Langevin (or random field) techniques are perhaps the most widely used approach in subsurface flow 
problems today. Stochastic flow and constitutive property variables are represented by a mean plus a random fluctuation function (or "equivalent noise source" function) in Langevin approaches. As a simple example, consider a two-phase system of oil and water in isotropic media where sources, sinks, capillary pressure, and gravity effects are neglected. From Darcy's law, the total fluid velocity, V(x), is expressed in a stochastic version of the Buckley - Leverett formulation (Appendix E) as

$$
\mathrm{V}(\mathrm{x})=-\mathrm{M}(\mathrm{x}) \bullet[\mathrm{K}(\mathrm{x}) \bullet \nabla \mathrm{P}(\mathrm{x})] .
$$

The total fluid mobility, $\mathrm{M}(\mathrm{x})$, is taken to be a known function for present purposes. The absolute permeability, $\mathrm{K}(\mathrm{x})$, is a function of rock properties, which are not varying in time; and $\mathrm{P}(\mathrm{x})$ is the fluid pressure. The velocity is steady-state because changes in pressure equilibrate very rapidly relative to changes in fluid saturations. To develop a Langevin model based on Eq. (A-1), the fluid velocity and pressure are represented as

$$
\begin{array}{ll}
\mathrm{V}(\mathrm{x})=\langle\mathrm{V}(\mathrm{x})\rangle+\mathrm{V}^{\prime}(\mathrm{x}), & \left.<\mathrm{V}^{\prime}(\mathrm{x})\right\rangle=0, \\
\mathrm{P}(\mathrm{x})=<\mathrm{P}(\mathrm{x})\rangle+\mathrm{P}^{\prime}(\mathrm{x}), & \left.<\mathrm{P}^{\prime}(\mathrm{x})\right\rangle=0,
\end{array}
$$

where $\mathrm{V}^{\prime}(\mathrm{x})$ and $\mathrm{P}^{\prime}(\mathrm{x})$ are random fluctuation functions and angle brackets denote statistical averages. Similarly, absolute permeability is represented as the random variable,

$$
\mathrm{K}(\mathrm{x})=<\mathrm{K}(\mathrm{x})>+\mathrm{K}^{\prime}(\mathrm{x}), \quad<\mathrm{K}^{\prime}(\mathrm{x})>=0 . \quad(\mathrm{A}-4)
$$

Random field methods focus largely on the evaluation of the fluctuation functions $\mathrm{V}^{\prime}(\mathrm{x}), \mathrm{P}^{\prime}(\mathrm{x})$, and $\mathrm{K}^{\prime}(\mathrm{x})$, as well as their second moments, making extensive use of geostatistical stipulations, perturbation techniques, and functional analysis ('estimator') techniques. When the random variables $\mathrm{V}(\mathrm{x}), \mathrm{P}(\mathrm{x})$, and $\mathrm{K}(\mathrm{x})$ take specialized normal or log-normal forms, they are completely defined by their means and varianccs, from which uncertainties and prediction errors are readily estimated. These specialized forms of distribution functions may not, however, accurately represent physical conditions in an actual reservoir system. In such instances, results regarding not only the physical state but also estimated uncertainties in parameter realizations could be misleading. Nonetheless, such statistically-based parameter estimation techniques generally invoke certain specializations (e.g., Gaussian or multi-Gaussian distributions, spatially constant means, homogeneity, small log-transmissivity variances, etc.) in order to make the calculation of parameter realizations tractable [Zimmerman et al, 1998]. Formation property realizations are usually conditioned by more or less systematic comparisons between measured pressures, permeability, and forward model calculations of pressure. But, owing to its illposedness, an inverse flow PDE for permeability (or transmissivity) is usually not explicitly solved and/or calibrated properly to permeability measurements. The odds are then overwhelming that estimated realizations of $\mathrm{K}(\mathrm{x})$ will fail to satisfy continuity constraints of 
the flow equation in the sense of the inverse solution, for reasons that will become more apparent in later sections. Excellent summaries, reviews and their cited references of many of the existing approaches can be found in works by McLaughlin and Townley [1996], Zimmerman, et al [1998], Dagan [1989], and Gelhar [1993].

\section{Moment PDE techniques}

An alternative stochastic approach is developed in terms of a statistical hicrarchy of evolution equations (PDEs) for averages of moments, e.g., mean, mean-square, and possibly higher moments and cross-moments of flow and constitutive property variables. The averaged moments are in fact physical observables that describe both the dynamical evolution and the statistical properties of physical systems ${ }^{3}$. Statistical moments in ground water and reservoir problems are usually truncated at second-order; and the resulting system of moment PDEs is to be directly solved and calibrated with measured data. But, first, several long-standing problems that have prevented successful solution and calibration of $\mathrm{F}$ I moment equations at the lowest statistical order require resolution if further progress is to be accomplished.

Direct solutions of inverse flow equations for mean aquifer transmissivity have been investigated over a span of at least forty years with various method-of-characteristics, fixedgrid finite difference and finite element techniques. (See, for example, articles by R. W. Nelson [1961, 1962], Emsellen and de Marsily [1971], Frind and Pinder [1973], and Dagan [1989].) Well-known problems associated with non-uniqueness, noise, and mathematical instability were not mitigated satisfactorily in these and other similar efforts. Several essential tools and techniques were lacking; they include:

- highly accurate dynamic adaptive-grid PDE solution methods, to help reduce or eliminate purely numerical sources of noise,

- simultaneous solutions of nonlinear forward and inverse PDEs, to better constrain realizations and mitigate mathematical instability,

- effective spatial filtering techniques, to help suppress noise in both pressure interpolations and derived pressure gradients,

- calibration techniques that respect basic sampling principles for sparse data and non-uniqueness,

- dynamic adaptive-grid finite element solutions of F-I flow equations, to interpolate transmissivity and pressure distributions, while also respecting measured datum points, and

- calibration techniques that respect Cauchy problem requirements in the inverse equation [John, 1982; Courant and Hilbert, 1953; Hadamard, 1952].

\footnotetext{
${ }^{3}$ Although not the main point of this discussion, the calibration of modeled physical observables to measurements generally requires fewer datum points than corresponding calibrations of modeled probability distribution functions to measurements. We have therefore not considered pdf approaches in this work. Interested readers may, however, wish to peruse the interesting work of Carle and Fogg [1996a,b], which describes a pdf transition probability technique for estimating properties of porous media.
} 
Recent advances in these six problem areas have apparently mitigated many of the classic barriers for solving ground water inverse problems with direct solutions of F-I moment PDEs [Gelinas et al,1998].

To develop the first-order moment equation for the oil-water example considered above; Equations (A-2) - (A-4) are substituted into Equation (A-1). Statistical averaging of the resulting equation gives

$$
<\mathrm{V}(\mathrm{x})>=-\mathrm{M}(\mathrm{x}) \bullet\left[<\mathrm{K}(\mathrm{x})>\bullet \nabla<\mathrm{P}(\mathrm{x})>+<\mathrm{K}^{\prime}(\mathrm{x}) \nabla \mathrm{P}^{\prime}(\mathrm{x})>\right] . \quad(\mathrm{A}-5)
$$

Without loss of generality, $M$ is taken to be identity for present purposes. For steady-state incompressible flow, the lowest-order PDE for flow in porous media with no sources or sinks is:

$$
\nabla \bullet<\mathrm{V}(\mathrm{x})>=0
$$

which can be written, using (A-5), as:

$$
\nabla \bullet[<\mathrm{K}(\mathrm{x})>\bullet \nabla<\mathrm{P}(\mathrm{x})>]+\nabla \bullet\left[<\mathrm{K}^{\prime}(\mathrm{x}) \bullet \nabla \mathrm{P}^{\prime}(\mathrm{x})>\right]=0 .
$$

In a convenient operator notation for moment PDEs, the first-order flow equation (A-7) is written as

$$
\mathrm{L}_{1}\left(\mathrm{~V}_{\mathrm{m}}\right)=\mathrm{Q}_{\mathrm{r}}
$$

The operator $L_{1}\left(V_{m}\right)$ represents the left-hand-side of the first-order moment equation. Functionally, it is the negative divergence of the mean Darcy velocity $\left(V_{m}\right)$, namely;

$$
\mathrm{L}_{1}\left(\mathrm{~V}_{\mathrm{m}}\right)=\nabla \cdot[<\mathrm{K}(\mathrm{x})>\bullet \nabla<\mathrm{P}(\mathrm{x})>]=-\nabla \cdot \mathrm{V}_{\mathrm{m}}, \quad(\mathrm{A}-9 \mathrm{a})
$$

where

$$
\mathrm{V}_{\mathrm{m}} \equiv-<\mathrm{K}(\mathrm{x})>\bullet \nabla<\mathrm{P}(\mathrm{x})>
$$

The operator $Q_{r}$ on the right-hand-side of equation (A-8) involves a second-order mixed moment of absolute permeability and pressure gradient fluctuations about their respective means, namely,

$$
\mathrm{Q}_{\mathrm{r}}=-\nabla \cdot\left\langle\mathrm{K}^{\prime}(\mathrm{x}) \bullet \nabla \mathrm{P}^{\prime}(\mathrm{x})\right\rangle \equiv \nabla \cdot \mathrm{V}_{\mathrm{r}}
$$

where the 'residual' velocity $V_{r}$ is defined by 


$$
\left.\mathrm{V}_{\mathrm{r}} \equiv-<\mathrm{K}^{\prime}(\mathrm{x}) \bullet \nabla \mathrm{P}^{\prime}(\mathrm{x})\right\rangle .
$$

The residual velocity is a (second-order) cross-correlation term that contributes to the rate of change of the mean flow equation. Using equations (A-9) and (A-10), equation (A-8) can readily be written as a conventional mean flow equation with an effective source term, $Q_{r}$, as

$$
-\nabla \bullet \mathrm{V}_{\mathrm{m}}=\mathrm{Q}_{\mathrm{r}}\left(\mathrm{V}_{\mathrm{r}}\right) .
$$

Owing to the presence of the second-order term $V_{r}$ in the mean flow equation (A-11), a second-moment PDE is generally needed for evaluating $Q_{r}$. Second-moment PDEs are frequently integro-differential equations, as can be seen in the article by Neuman and Orr [1993]. It serves our purpose here to again use operator notation, with spatial arguments suppressed, to represent the second-order moment PDE for $V_{r} \sim<K^{\prime} \bullet \nabla P^{\prime}>$ as

$$
\left.\left.\mathrm{L}_{2}\left(<\mathrm{K}^{\prime} \bullet \nabla \mathrm{P}^{\prime}\right\rangle\right)=\mathrm{f}_{2}(<\mathrm{K}\rangle,\langle\mathrm{P}\rangle,\left\langle\mathrm{K}^{\prime} \mathrm{K}^{\prime}\right\rangle,\left\langle\mathrm{P}^{\prime} \mathrm{P}^{\prime}\right\rangle\right),
$$

where third-order moments have been truncated. The right-hand-side of this equation (A12 ) is a complicated function $\left(f_{2}\right)$ of the arguments indicated. Because Eqn. (A-12) depends upon second-order correlations, $\left\langle\mathrm{K}^{\prime} \mathrm{K}^{\prime}\right\rangle$ and $\left\langle\mathrm{P}^{\prime} \mathrm{P}^{\prime}\right\rangle$ the second-order hierarchy is completed by the PDEs for $\left\langle\mathrm{K}^{\prime} \mathrm{K}^{\prime}\right\rangle$ and $\left\langle\mathrm{P}^{\prime} \mathrm{P}^{\prime}\right\rangle$, namely,

$$
\begin{aligned}
& \left.\left.\mathrm{I}_{3}\left(<\mathrm{K}^{\prime} \mathrm{K}^{\prime}\right\rangle\right)=\mathrm{f}_{3}(<\mathrm{K}\rangle,\langle\mathrm{P}\rangle,\left\langle\mathrm{K}^{\prime} \mathrm{P}^{\prime}\right\rangle,\left\langle\mathrm{P}^{\prime} \mathrm{P}^{\prime}\right\rangle\right), \\
& \left.\left.\mathrm{L}_{4}\left(<\mathrm{P}^{\prime} \mathrm{P}^{\prime}\right\rangle\right)=\mathrm{f}_{4}(<\mathrm{K}\rangle,\langle\mathrm{P}\rangle,\left\langle\mathrm{K}^{\prime} \mathrm{P}^{\prime}\right\rangle,\left\langle\mathrm{K}^{\prime} \mathrm{K}^{\prime}\right\rangle\right) .
\end{aligned}
$$

To briefly summarize, the system of first- and second-moment PDEs is comprised of Equations (A-11) - (A-14). Several properties of the first-order flow equation are immediately evident:

- If $Q_{r}$ is negligible, the mean flow equation (A-11) can be directly solved and calibrated to measured data if wellfield source terms and boundary conditions are known or stipulated to be statistically independent. In this instance, statistical properties can be evaluated separately from the mean flow PDE.

- If the cross-correlation term $\left(V_{r}\right)$ in $Q_{r}$ is Fickian (i.e., proportional to a mean coefficient times $\nabla\langle\mathrm{P}\rangle$ ), solution of the moment PDE hierarchy is again separable in the sense that the mean flow equation (A-11) reduces to

$$
\nabla \bullet[<\tilde{\mathrm{K}})>\bullet \nabla<\mathrm{P}>]=0,
$$

which resembles the conventional source-free mean flow PDE with permeability $<\tilde{\mathrm{K}}>$. In these two instances, with $Q$, equated to either zero or a Fickian function, the higher-order 
moments may depend on the mean variables, but the mean variables do not depend on the higher-order moments. The mean and higher-order moments can then be calibrated to measured data by separate techniques without loss of generality.

- If the cross-correlation term $\left(V_{r}\right)$ is believed to be known independently, e.g., from other geostatistical analyses, the PDE hierarchy may again be solved in a separable manner, using the F-I methods to solve and calibrate the mean flow equation (A-11), with $Q_{r}$ given from other analyses.

- Finally, when the permeability and pressure are significantly correlated, simultaneous solution and calibration of the coupled equations and (A-11) - (A-14) are generally required for the best results. General solutions of the moment PDE hierarchy are difficult to obtain in practice because the second-order moment PDEs (A-12) - (A-14) are usually nonlinear integro-differential equations [Neuman and Orr, 1993]. It is possible, but not yet clear, that extended adaptive-grid techniques will fare significantly better than the specialized transform and Monte Carlo techniques used to date for the solution of integrodifferential PDEs.

Regardless of the significance of residual flux, $Q_{r}$, appropriate sparse-data calibration techniques for the forward and inverse solutions of the lowest-order flow equation (A-11) are of overriding importance. For it is at low-order where the classic problems of illposedness and mathematical instability reside. All stochastic inverse methods, including widely-used manual history-matching, are dependent upon the quality of the low-order simulations. Hence we have emphasized in this report only the lowest-order flow equation (A-11) for cases in which $Q_{r}$ is either Fickian or negligible. 


\section{Appendix B: Analytic Description of the F-I Method}

\section{Simultaneous Conditioning of Forward and Inverse Flow Models}

In the interest of economy, this Appendix abstracts pertinent parts of the mathematical development of the new F-I modeling techniques from their original documentation in an LLNL report by Gelinas et al [1998]. The context of the original developments was for single-phase flow in saturated porous media for ground water remediation problems. These techniques are being extended to F-I modeling of 2-D transient oil-water problems in this Phase I project, where the primary emphasis is on proofs of concepts. Analytic descriptions of the simultaneous F-I modeling techniques are presented here in their original notation pertaining to ground water aquifer problems. Additionally detailed mathematical proofs and text will be found in journal articles when they become generally available.

To emphasize basic concepts without significant loss of generality, steady-state ground water flow in a confined aquifer is modeled according to Darcy's law over regional scales. The steady-state flow equation in three spatial dimensions (3-D) can be written as:

$$
\nabla \cdot(\mathrm{K} \nabla \mathrm{h})+\mathrm{Q}=0,
$$

where $\mathrm{K}$ is the hydraulic conductivity of the aquifer, $\nabla \mathrm{h}$ is the hydraulic head gradient, and $\mathrm{Q}$ represents internal source and sink rates. When the aquifer thickness is substantially smaller than the horizontal scale of the flow domain, the two-dimensional (2-D) flow equation,

$$
\nabla \bullet(\mathrm{T} \nabla \mathrm{h})+\mathrm{Q}=0,
$$

is a reasonable approximation for many aquifers. The transmissivity $\mathrm{T}$ is defined as the product of $\mathrm{K}$ and the aquifer thickness, $\mathrm{b}$. (Unless otherwise noted, we exercise an understood convention in subsurface flow articles of using scalar notation for tensor quantities, e.g., $\mathrm{K}$ and $\mathrm{T}$ in this report.) $\mathrm{A}$ site characterized by borehole measurements of hydraulic head and transmissivity is depicted schematically in Figure B1. Hydraulic head and transmissivity are measured at discrete locations in the domain, $\Omega$, and on its boundaries $\partial \Omega$. The thickness of the aquifer is assumed to be known from measurements, as are pumping sources and sink rates in equations (B-1) and (B-2). Transmissivity data are usually more sparse than head data. Head measurements are usually much more accurate and localized at the borehole than are transmissivity values. For convenience in this initial work, Q is henceforth taken to be zero. 


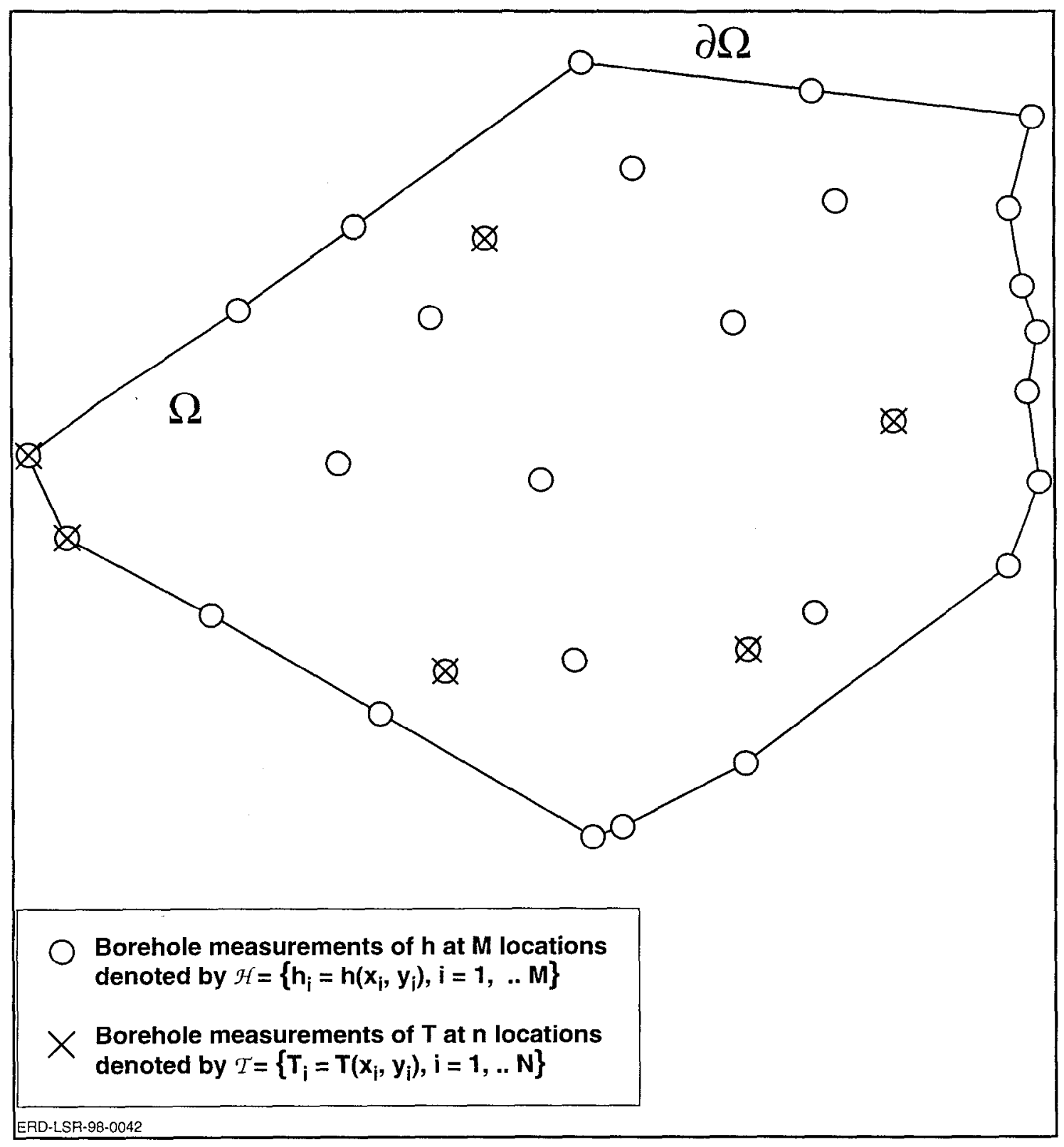

Figure B-1: Remediation site domain $\Omega$ with boundary $\partial \Omega$, characterized by discrete borehole measurements of $h$ and $T$.

The main task is to determine transmissivity distributions everywhere in a domain $\Omega$ based on knowledge of measured hydraulic head and transmissivity at a discrete and finite subset of $\Omega$ (see Figure 1). The data is assumed to fairly conform with the model assumptions of a steady-state condition of an aquifer region with no internal ground water sources or sinks. The case with non-zero sources/sinks is being developed in separate work. Because the present work focuses on the mathematical model for the lowest-order (mean) equations of the moment hierarchy, the angle brackets for statistical averages in the 
text will henceforth be dropped for convenience. Also, to facilitate subsequent discussions, the following definitions are introduced here, referring to Figure B1.

$S \quad$ A finite set of 2-D Cartesian coordinates $\left\{\left(\mathrm{x}_{\mathrm{i}}, \mathrm{y}_{\mathrm{i}}\right), \mathrm{i}=1,2, \ldots\right\}$ at which measurements of hydraulic head are collected. This 'fixed point set' becomes part of the nodal assembly in the finite element solution of the governing PDEs.

$\mathcal{H}$ The set of measured head values on the set $S$.

$\mathcal{H}^{\prime} \quad$ Is constructed from $\mathscr{T}$ by replacing all elements of $\mathscr{H}$ by those obtained from the somewhat smoothed solution $h_{s}$ in equation (B-9).

$\tau$ A set of transmissivities inferred from other well tests at some subset of $S$. These will be referred to as transmissivity measurements.

$\Omega \quad$ A domain in the $\mathrm{x}-\mathrm{y}$ plane over which one attempts to solve for $\mathrm{h}$ and $\mathrm{T}$ and whose boundary $\partial \Omega$ is a polygon consisting of those linear segments joining the 'outermost' points of the set $S$.

$S \quad$ All points of $S$ which are not included in $\partial \Omega ; S^{\prime}=S-\partial \Omega$.

$\Omega^{\prime} \quad$ The entire domain excluding the set $S ; \Omega^{\prime}=\Omega-S$.

$\mathrm{C}^{\mathrm{n}}(\Omega) \quad$ The class of all continuously differentiable functions in $\Omega$ up to order $\mathrm{n}$.

Notice that it is always possible to construct $\Omega$ so that it is the convex hull of $S$. However, such smallest convex set containing the set $S$ could be inadequate in specific instances that are described in forthcoming journal articles.

Starting from the mean flow equation for porous media with physical sources and sinks of ground water $(Q)$ equal to zero,

$$
\nabla \bullet(\mathrm{T} \nabla \mathrm{h})=0
$$

and assuming that $\mathrm{h}(\mathrm{x}, \mathrm{y})$ is known everywhere in $\Omega$, then equation (B-3) can in principle be solved for $\mathrm{T}(\mathrm{x}, \mathrm{y})$ as the unknown dependent variable. This is the inverse solution process for $\mathrm{T}$ and is to be contrasted with the familiar usage of equation (B-3) to solve a forward problem for $h$ when $T$ is given everywhere in $\Omega$. Frequently, equation (B-3) is referred to as the inverse equation when the inverse solution process is being considered; its solution, $\mathrm{T}$, is then referred to as the inverse solution. Being a first order PDE in T, equation (B-3) can be solved by the method of characteristics provided that its coefficients satisfy some reasonable smoothness conditions. Although the classic characteristic approach is not employed in this work; the latent wealth of information pertaining to the theory of first order partial differential equations [Courant and Hilbert, 1953; John, 1982] is nonetheless employed extensively and implicitly in the present work.

Sparsely measured head data (as well as sparse transmissivity data) present both the major problem and the reason for transmissivity parameter estimation. Because of the lack of complete knowledge of h everywhere in $\Omega$, it follows, that head gradients are not defined in all of $\Omega$; and thus a PDE simply does not exist from which $\mathrm{T}$ can be determined. An 
immediate way to proceed, then, is to find an interpolating surface that passes through all of the head datum points. Because a vast number of such surfaces often exist, one finds that, unless further constraints are imposed, the inverse solution (if found) will often yield unphysical transmissivity distributions. The cause of this difficulty is readily explained: most trial interpolating surfaces that pass through the measured head datum points form relative maxima or minima 'spikes' on the set $S$, thereby violating the maximum-principle for elliptic differential equations. See, for example, Protter and Weinberger [1984]. The interpolating surfaces must therefore be selected judiciously-which is the starting point for the developments in this work.

For readers who may need a "refresher", it is important to review briefly the method of characteristics for first-order quasi-linear differential equations. In order to write equation (B-3) in standard form, one assumes, momentarily, that the head, $h$, belongs to the class of functions $C^{2}(\Omega)$. The theory of characteristics then leads one to solve the following set of autonomous ordinary differential equations (ODEs):

$$
\begin{gathered}
\frac{d x}{d s}=\frac{\partial h}{\partial x} \\
\frac{d y}{d s}=\frac{\partial h}{\partial y} \\
\frac{d T}{d s}=-T \Delta h
\end{gathered}
$$

where $s$ is a parameter measured from some given 'initial' point $\left(\mathrm{x}_{0}, \mathrm{y}_{0}, \mathrm{~T}_{0}\right)$, and $\Delta$ denotes the Laplacian operator. The first two ODEs (B-4) and (B-5) easily map the geometry of characteristic curves (or more precisely, the projection of the characteristic curves on the $x-y$ plane) based solely on knowledge of head gradients. The third ODE determines $T$ uniquely along the entire characteristic which passes through $\left(\mathrm{x}_{0}, \mathrm{y}_{0}\right)$ and such that $\mathrm{T}=\mathrm{T}_{0}$ at $\mathrm{s}=0$. In fact, if the parametric solutions: $\mathrm{x}=\mathrm{x}\left(\mathrm{s}, \mathrm{x}_{0}, \mathrm{y}_{0}\right), \mathrm{y}=\mathrm{y}\left(\mathrm{s}, \mathrm{x}_{0}, \mathrm{y}_{0}\right)$ obtained from equations $(\mathrm{B}-4)$ and (B-5) are substituted into equation (B-6), the solution to equation (B-6) is obtained readily in the form

$$
\mathrm{T}=\mathrm{T}_{0} \exp \left\{-\int_{0}^{\mathrm{s}}\{\Delta \mathrm{h}(\tau) \mathrm{d} \tau\}\right.
$$

which incidentally guarantees that $\mathrm{T}$ will always remain positive, as expected. It is assumed in equation (B-7) that the functional form of $\Delta h$ is known at least along the characteristic curve through $\left(\mathrm{x}_{0}, \mathrm{y}_{0}\right)$. Transmissivities would be completely determined everywhere in $\Omega$ once $\mathrm{T}$ is given values along an entire non-characteristic initial curve $\Gamma$, provided that all of the characteristic curves emanating from $\Gamma$ sweep the entire domain $\Omega$. If not, one can only solve for $\mathrm{T}$ on the subdomain that is swept out by the characteristic curves.

When dealing with the reality of non-smooth $\mathrm{h}$, several daunting problems and conccrns must be addressed. The first problem, the satisfactory resolution of head Laplacians from sparse data, is in practice intractable because the head gradients are often discontinuous on 
$\Omega$; and the head Laplacian is therefore undefined on all of $\Omega$. A second concern is that only one transmissivity value is needed on a characteristic curve in order to determine all other transmissivity values along that curve uniquely. If additional inconsistent values were to be assigned somehow on the same characteristic curve, as is frequently done in stochastic transmissivity parameterizations, zonation schemes, and history-matching techniques, fundamental ODE solution requirements for the inverse equation are contradicted and physical continuity is not respected in the inverse sense.

A third problem is one of managing logistics; i.e., the bookkeeping of all generated characteristic curves emanating from some finite set of points traversing $\Gamma$ for the eventuality of constructing the integral surface containing these characteristic curves. Thoughts concerning these problems motivated the present quest for more viable parameter estimation techniques-techniques that can solve simultaneous forward and inverse flow PDEs, augmented by spatial filtering PDEs for data smoothing, in a true physical continuum according to kinetic theory.

The process developed here for heading off some of the classic difficulties mentioned above is composed of four basic steps. Each step can be described and verified separately before merging them finally into a simultaneous, iterative process for sparse, noisy data in field applications. The basic steps are:

1. Form a trial interpolating surface $\mathrm{h}_{\mathrm{r}}(\mathrm{x}, \mathrm{y})$ in $\Omega$ such that, on the discrete subset $S^{\prime}$ of $\Omega, h_{\mathrm{r}}$ is forced to take on the corresponding measured values in the set $\mathcal{H}$. The interpolator employed here is a variant of the flow equation.

2. Introduce and solve an additional PDE for smoothing $h_{r}$ in $\Omega$. The amount of smoothing of spikes in $\mathrm{h}_{\mathrm{r}}$ at measurement points is controlled by user-selected parameters. This produces spatially filtered distributions $h_{s}$ that can deviate from $\mathscr{H}$ on the set $S^{\prime}$ by any preset amount.

3. Introduce and solve two additional PDEs that produce smoothed head gradient components, $u$ and $v$, from $h_{\mathrm{c}}$ obtained above. It is extremely important to smooth head gradients prior to using them as coefficients in the inverse equation.

4. Solve a regularized variant of the inverse equation (B-3) using a spatially filtered head gradient $(\mathrm{u}, \mathrm{v})$ in lieu of $\nabla \mathrm{h}$.

The first step is a 'rough' interpolation of the hydraulic head data. The key idea is to bring a stronger influence of physical dynamics to bear on the interpolation than is usually applied in geostatistical interpolation methods. An obvious choice is to use a variant of the mean flow PDE, itself, as the interpolator. Letting $\mathrm{T}$ be a constant average value (or any constant value), the interpolating equation is

$$
\nabla \bullet\left(T \nabla h_{r}\right)=0 .
$$

It is important to note that equation (B-8) is to be satisfied in $\Omega$ ', rather than in the entire domain $\Omega$. The solution $\mathrm{h}_{\mathrm{r}}$ is 'pinned,' or 'clamped', to the set $\mathcal{H}$ on the remaining set $S$. In other words, $\mathrm{h}_{\mathrm{r}}$ is constrained to both respect the values of measured head and to satisfy the flow equation everywhere else in $\Omega$. This is directly analogous to solving mechanical stress problems with fixed loads at given coordinates. Galerkin numerical solution methods 
are especially well-suited for solving these types of PDEs because they can respect the data at fixed points and yield optimized solutions that minimize PDE residuals over the rest of the problem domain. On $\partial \Omega$, Dirichlet boundary conditions are assigned to $h_{r}$. Linear interpolation is expedient but other methods of assignment may be used when they are more appropriate.

This first trial interpolating surface is calculated in this work by employing a dynamic adaptive grid finite element code toolkit, FlexPDE, which enables the user to solve equation (B-8) in $\Omega^{\prime}$, subject to the pinning constraints. (This feature was implemented in our version of the FlexPDE toolkit by its author, R.G. Nelson, specifically for development of the present inverse techniques. See the texts of Backstrom $(1994,1998)$ for not only specific information about using such advanced toolkits as PDEase and FlexPDE but also new modes of posing computational models with these emerging tools in order to respect and enforce essential mathematical requisites in complex physical problems.)

The solution $h_{r}$ in this step is expected to possess relative maxima or minima that may be associated with several possible origins. First is the sweeping approximation of replacing $T$ by its average value on $\Omega$. While this might seem to be the major cause for such extraneous behavior, it is seldom the case. Other possible causes include: data errors or noise, ground water sources/sinks that were previously unknown, absence of a perfect steady-state in nature, and local/non-Darcy effects (equivalent sources).

The second step performs an additional smoothing process on $h_{\mathrm{r}}$ over $\Omega$ in order to damp or completely diminish spurious spikes. It is necessitated because complete clamping of $\mathrm{h}_{\mathrm{r}}$ to $\mathscr{H}$ on $S$ can create a corresponding set of local spikes, which cannot exist anywhere in $\Omega$, in the absence of physical sources or sinks. (When such spikes actually turn out to be previously unknown physical sources or sinks, the presently described techniques turn out to be useful 'source-finders,' which is another subject that will be considered as part of 'data-mining' in future work.) Looking ahead to the next (third) step, much of the important information content from hydraulic head measurements, no matter how sparse or devoid of high spatial frequency information they may be, resides in the gradients and Laplacians of the head, as was indicated in previous discussion. The objective in the second and third steps is to extract as much information as possible by performing spatial filtering that is commensurate with the spatial intervals between the measured data [Bracewell, 1986]. Such filtering is also required to produce a smoothed $h_{s}$ that effectively satisfies the maximum principle for elliptic differential equations. The clamping imposed on $h_{r}$ is therefore relaxed at the measurement points by solving the following PDE for $h_{s}$ on $\Omega$ :

$$
\Delta \mathrm{h}_{\mathrm{s}}+\alpha\left(\mathrm{h}_{\mathrm{r}}-\mathrm{h}_{\mathrm{s}}\right)=0 \quad \text { in } \Omega .
$$

The Laplacian operation in the first term of equation (B-9) is recognized to be a bandpass filter function [McGillem and Cooper, 1984]. It does not have a sharp cut-off at any spatial frequency; so some latitude can be exercised in Laplacian smoothing with this factor in mind. The second term in equation (B-9) can be viewed as a penalty function that controls the amount of smoothing of $h_{r}$ through proper choices of the parameter $\alpha$. Clearly, very large $\alpha$ yields an $h_{s}$ that hardly differs from $h_{r}$; that is, very little smoothing is done. And vice versa, as $\alpha$ approaches zero, $h_{s}$ approaches a harmonic solution in $\Omega$. But too 
much smoothing is obviously undesirable when it loses (by aliasing) significant amounts of information about the set $\mathcal{H}$ on $S$ '. Criteria for the extent of smoothing in this step are discussed in technical journal submissions. (Recall that the average transmissivity assumed in the first step is not the final distribution that is sought.) The solution $h_{s}$ is then used to construct a new set $\mathcal{H}^{\prime}$ of smoothed heads on $S^{\prime}$, and $\mathscr{H}^{\prime}$ replaces $\mathscr{H}$ in subsequent steps.

The rationale for step three is built on the fact that the gradient of $h_{s}$, and not $h_{s}$ per se, is the critical determinant in solving for $T$ in step four. Because small deviations of $h_{s}$ from a correspondingly true head produce large deviations in calculated head gradient, this smoothing step is mandatory. The principle applied in the third step is similar to that in step two. Defining $(\mathrm{u}, \mathrm{v})$ to be the desired smoothed head gradients, relative to less-smooth gradients $\left(\partial \mathrm{h}_{\mathrm{s}} / \partial \mathrm{x}, \partial \mathrm{h}_{\mathrm{s}} / \partial \mathrm{y}\right)$, the following two PDEs are introduced and solved for on $\Omega$ :

$$
\begin{aligned}
& \Delta \mathrm{u}+\beta\left(\frac{\partial \mathrm{h}_{\mathrm{s}}}{\partial \mathrm{x}}-\mathrm{u}\right)=0 \quad \text { in } \quad \Omega \\
& \Delta \mathrm{v}+\beta\left(\frac{\partial \mathrm{h}_{\mathrm{s}}}{\partial \mathrm{y}}-\mathrm{v}\right)=0 \quad \text { in } \quad \Omega .
\end{aligned}
$$

The options used for assigning boundary conditions (BCs) for $\mathrm{u}$ and $\mathrm{v}$ on $\partial \Omega$ are straightforward and are specifically discussed in journal submissions.

The fourth step attempts to determine $T$ based on knowledge of spatially filtered head gradient $(\mathrm{u}, \mathrm{v})$ obtained in step three. As part of a procedure that deals mainly with solutions of boundary value problems (BVPs) in steps $1-3$, it is both desirable and advantageous to recast the inverse equation so that a BVP can be prescribed here as well. This is accomplished by solving a regularized version of equation (B-3) through the addition of the regularizing term $\varepsilon \Delta \mathrm{T}$, for sufficiently small $\varepsilon$,

$$
\nabla \bullet(\mathrm{Tu}, \mathrm{Tv})+\varepsilon \Delta \mathrm{T}=0 \quad \text { in } \quad \Omega \text {. }
$$

This modified equation (B-12) is in principle an elliptic equation. But in practice it is essentially a hyperbolic PDE. The solution to equation (B-12) can then be completely determined, provided that $T$ is given along some non-characteristic curve $\Gamma$ in the closure of $\Omega$ and such that the continuum of characteristics emanating from $\Gamma$ span the entire domain $\Omega$. For brevity, a $\Gamma$ curve that satisfies these conditions will be referred to as an admissible $\Gamma$ curve. If no admissible $\Gamma$ curve exists for the entire domain $\Omega$, then one must partition $\Omega$ into two or more subdomains, each of which possesses its own admissible $\Gamma$ curve. Fortunately, in the absence of sources and sinks in ground water flow problems, the geometry of characteristic curves is usually simple enough (but not trivial!) that it only requires the specification of a single admissible $\Gamma$ curve. No further specification of $T$ along the remainder of $\partial \Omega$ is required. Such a 'no specification' boundary condition is exacted by recycling integrands in all boundary integrals that are produced from integration by parts in the numerical solution process [Oden et al, 1986]. That is, whatever expression appears in a boundary integral, it is reused, as is, in forming the mass matrix of the Galerkin equations. 
It appears, so far, that knowledge about the projection of the characteristic curves (or simply characteristics) on the $\mathrm{x}-\mathrm{y}$ plane for equation (B-12) with $\varepsilon=0$, enters in this analysis for the end-purpose of determining admissible curves $\Gamma$. Such a purpose could just as well be accomplished quickly by graphically investigating the normalized spatially filtered head gradient $(\mathrm{u} / \mathrm{s}, \mathrm{v} / \mathrm{s}), \mathrm{s}=\left(\mathrm{u}^{2}+\mathrm{v}^{2}\right)^{1 / 2}$ (i.e., a vector field plot). Knowledge about the characteristics, and more importantly how stably and efficiently they are determined, actually serve a broader goal in this work. Consider, then, the solution $\psi$ of the following first order PDE:

$$
\mathrm{u} \frac{\partial \psi}{\partial \mathrm{x}}+\mathrm{v} \frac{\partial \psi}{\partial \mathrm{y}}=0 \quad \text { in } \quad \Omega
$$

and suppose $\Gamma$ is an admissible curve in $\Omega$, which could be a part of $\partial \Omega$. For simplicity, let $\psi$ take on any monotonically increasing set of values along $\Gamma$, say for instance $\psi=s, s$ being some parameterization of $\Gamma$ such that $0 \leq s \leq 1$ (see Figure B2). One can conclude from the theory of characteristics that the continuum of curves $\{\psi=C, 0 \leq C \leq 1\}$ is precisely the set of all characteristic curves for equation (B-13). That is, if one can solve equation (B-13) 'directly' in $\Omega$ in lieu of actually solving the standard ODEs for the characteristic curves, namely,

$$
\frac{\mathrm{dx}}{\mathrm{ds}}=\mathrm{u}, \frac{\mathrm{dy}}{\mathrm{ds}}=\mathrm{v},
$$

the entire geometry of characteristics is then obtained, all at once, from the knowledge of $\psi$. Such direct solution of equation (B-13) can be accomplished by regularization with the term $\varepsilon \Delta \psi$, paralleling the previous discussion. The essential $\mathrm{BC}$ required here is that which is given along $\Gamma$, namely $\psi=\mathrm{s}$; and 'no specification' $\mathrm{BC}$ is required along the remainder of $\partial \Omega$. The function $\psi$ should not be confused with the classical stream function, which is usually defined as a solution of the Cauchy-Reimann equations and is obtained as the solution of a harmonic equation with proper choices of $\mathrm{BC}$ on $\partial \Omega$. Classical stream line solutions obtained in this way are identical to $\psi$ only for constant $\mathrm{T}$. 


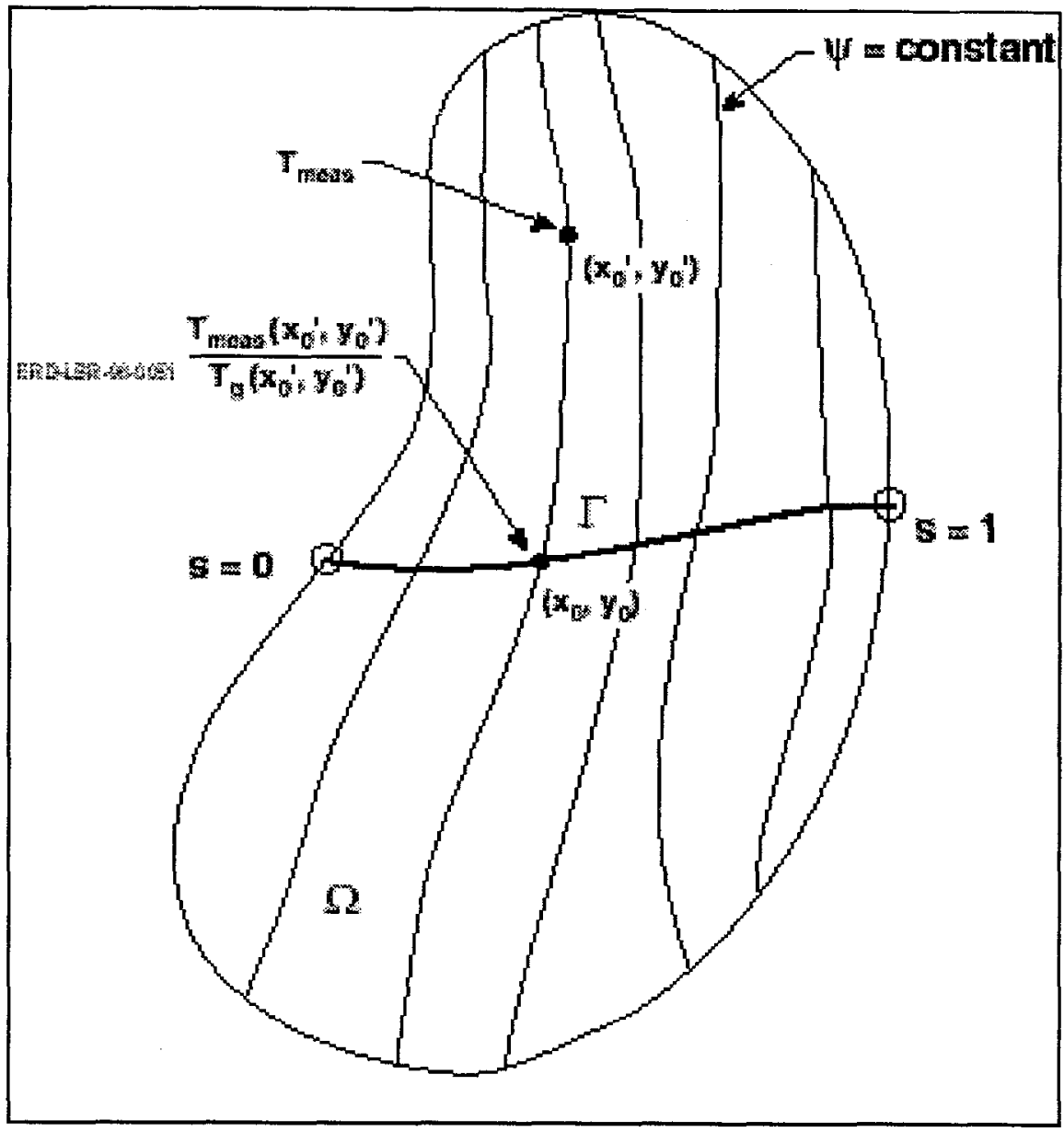

Figure B-2: Schematic representation of an admissible $\Gamma$ curve in a domain $\Omega$. Transmissivity values measured at $\left(\mathrm{x}_{0}{ }^{\prime}, \mathrm{y}_{0}{ }^{\prime}\right)$ can be projected to the $\Gamma$ curve by procedures developed in journal article submissions.

Returning to equation (B-12), it is of interest to note its relationship to equation (B-13). For simplicity assume that both $u$ and $v$ are differentiable. Equation $(B-12)$ can then be written for $\varepsilon=0$ as,

$$
u \frac{\partial T}{\partial x}+v \frac{\partial T}{\partial y}+T\left(\frac{\partial u}{\partial x}+\frac{\partial v}{\partial y}\right)=0
$$

In the absence of the last term (which is essentially $T \Delta h$ ), equation (B-15) is identical to equation (B-13) with $\psi$ replaced by $\mathrm{T}$. The point to be made here is that the behavior of the solution process in (B-13) is expected to reflect on how the solution process to (B-15) will develop. In fact, it was found that it is often convenient to represent $T$ in $(B-12)$ as the product of two functions,

$$
\mathrm{T}=\Phi \bullet \mathrm{T}_{\mathrm{g}}
$$


where $T_{\mathrm{g}}$ is a general or generic solution to equation $(\mathrm{B}-12)$ with $\mathrm{T}_{\mathrm{g}}=1$ on $\Gamma$; and $\Phi$ is a particular solution to equation (B-13) with $\Phi=\mathrm{T}_{\gamma}$ along $\Gamma$. It is clear from this specification that $\mathrm{T}=\mathrm{T}_{\gamma}$ on $\Gamma$. That the factorized form of $\mathrm{T}$ in equation (B-16) formally satisfies (B-12) is also straightforward to deduce. One can further notice that $(u, v)$ only needs to be continuous along $\Gamma$ in order for the representation (B-16) to make sense. Also notice that, if $\Gamma$ is either a portion of the domain boundary $\partial \Omega$ or if it is an isocontour $h(x, y)=$ constant, then no assumptions about the smoothness of $(\mathrm{u}, \mathrm{v})$ along $\Gamma$ are required. The additional rationale for the $\mathrm{T}$ factorization will be addressed shortly.

The foregoing analysis of the four main steps was presented as sequential steps so that each succeeding step builds on the results of the preceding one, but not vice-versa. The core algorithm presented in this work, however, consists of merging, with slight modification, the equations studied in these steps into a full-fledged system of coupled nonlinear equations. The purpose of the sequential presentation was to: (i) better understand the motivations and rationale leading to the creation and execution of each step; (ii) derive a new set of head values $\mathcal{H}^{\prime}$ that are more compatible with prospective transmissivity distributions than $\mathcal{H}$; and (iii) obtain a reasonably good starting set of trial values for the quartic $\{h, u, v, T\}$.

The system of equations employed for the final determination of $\mathrm{T}$ is derived with few minor modifications from the four steps discussed previously. The system solved in this final stage consists of four PDEs in four unknowns $\{h, u, v, T\}$ expressed as:

$$
\begin{gathered}
\nabla \bullet \mathrm{T} \nabla \mathrm{h}=0, \\
\Delta \mathrm{u}+\alpha\left(\frac{\partial \mathrm{h}}{\partial \mathrm{x}}-\mathrm{u}\right)=0, \\
\Delta \mathrm{v}+\alpha\left(\frac{\partial \mathrm{h}}{\partial \mathrm{y}}-\mathrm{v}\right)=0, \\
\nabla \bullet(\mathrm{Tu}, \mathrm{Tv})+\beta \Delta \mathrm{T}=0 .
\end{gathered}
$$

The main distinction between equations (B-17) and the previous individual steps is that equation (B-8) with constant average $\mathrm{T}$ in step $\mathrm{l}$ is replaced by equation (B-17a), with both $\mathrm{T}$ and h unknown. A similar distinction holds between equation (B-17d) and equation (B12). Whereas equation (B-17d) treats $T, u$, and $v$ as unknowns, equation (B-12) was solved only for $\mathrm{T}$ with $\mathrm{u}$ and $\mathrm{v}$ assumed known. Notice that no further smoothing of $\mathrm{h}$, itself, is performed here. The smoothing of head gradients is however, retained. Discussions related to boundary conditions in the previous steps carry over to this system of coupled equations, with one exception. Namely, the set $\mathcal{H}$ in step one is replaced by the set $\mathcal{H}^{\prime}$ here. Taken as a whole, the system of PDEs (B-17) is obviously nonlinear in the unknown variables $\{\mathrm{h}, \mathrm{u}$, $\mathrm{v}, \mathrm{T}\}$. As such, it is important to start with good initial trial estimates according to the procedures developed in this work for starting the Newton-Raphson linearization process employed in the numerical PDE solver. 
As discussed previously for equation (B-16), one can factorize $\mathrm{T}$ as $\mathrm{T}=\Phi \cdot \bullet^{\prime} \mathrm{T}_{\mathrm{g}}$ and solve the system (B-17) with $\mathrm{T}_{\mathrm{g}}$ in place of $\mathrm{T}$, obtaining solutions for $\mathrm{u}, \mathrm{v}$, and $\mathrm{T}_{\mathrm{g}}$. One can then obtain $\Phi$ by solving separately, the following regularized PDE:

$$
\mathrm{u} \frac{\partial \Phi}{\partial \mathrm{x}}+\mathrm{v} \frac{\partial \Phi}{\partial \mathrm{y}}+\varepsilon \Delta \Phi=0
$$

The system (B-17) with $\mathrm{T}$ replaced by $\mathrm{T}_{\mathrm{g}}$ is devoid of any particular assignment of transmissivity data along $\Gamma$. (Recall that $T_{\mathrm{g}}=1$ along $\Gamma$ under this factorization.) The system (B-17) therefore needs to be solved only once; and many different possible realizations of $\mathrm{T}$ along $\Gamma$ can then be tested quickly by solving the simpler, single equation (B-18). The facility of $\mathrm{T}_{\mathrm{g}}=1$ on $\mathrm{T}$ implies that a reasonable initial guess for the NewtonRaphson linearization could be taken as $\mathrm{T}_{\mathrm{g}}=1$ everywhere in $\Omega$. On the other hand, an assignment of highly varying $T$ along $\Gamma$ when solving the original unfactorized $T$ can lead to instabilities and outright divergence of the numerical solution process.

\section{Undersampling Effects}

The primary challenge of inverse modeling is to deal with the daunting problems associated with determining transmissivity realizations (images) between boreholes from sparsely sampled data, so that the realizations obtained will agree as closely as possible with the actual spatial distribution that exists in nature. The realizations sought must respect all applicable principles of mathematics, physics, and signal processing. For testing groundtruth notice that, if an inverse solution algorithm is somehow given a set of practically perfect (very highly-resolved) discrete data for transmissivity and head from a well-posed problem, the algorithm has no way of knowing that the problem was abstracted from one with a unique solution. So a first test of any inverse method/algorithm is that it should produce a close semblance of the unique transmissivity distribution when such a data set is provided. That is the type of test used here to establish a connection with ground-truth. Once that is established, the effects of sparse data sampling can be examined gainfully.

Highly accurate adaptive-grid PDE tools enable one to construct ground-truth data sets for well-posed problems that do not have analytic solutions. The following such problem is constructed here with discontinuous transmissivity: A domain $\Omega$ consisting of the square ($4 \leq \mathrm{x} \leq 4)$ by $(-4 \leq \mathrm{y} \leq 4)$ is selected. As shown in Figure $\mathrm{B}-3, \Omega$ is partitioned into 64 equal blocks, or zones, of unit squares; and $\mathrm{T}$ is assigned a constant value on each of these 64 zones. Notice that the constant zonal values of $\mathrm{T}$ alternate several times between increasing and decreasing, geometrically, giving rise to a haphazard staircase-like shape with a range of $\mathrm{T}$ between 1 and about 75. Given this distribution for transmissivity in $\Omega$, the following boundary value problem (BVP) for $h$ is solved numerically: $\nabla \bullet(T \nabla h)=0$ in $\Omega$, along with the $B C s h=100$ on the side $x=-4 ; h=10$ on the opposite side, $x=4$, and zero flux condition along the remaining sides $y= \pm 4$. The solution to this BVP was obtained to a very high accuracy, using the PDEase toolkit. The solution obtained for the pair $(\mathrm{T}, \mathrm{h})$ is shown in Figure B-4 as head isocontours. To verify ground-truth of the inverse solution, a very dense set of discretely sampled datum points was then abstracted from the head 
isocontours in Figure B-4, along with a dense set of discretely sampled transmissivity datum points only on the Cauchy line, taken to be along the vertical line at $x=-4$. Provided with these sets of dense datum points, the F-I algorithms described previously in this Appendix calculated a transmissivity solution that was indistinguishable from the original distribution shown in Figure B-3. With ground-truth established, the effects of data undersampling are examined next.

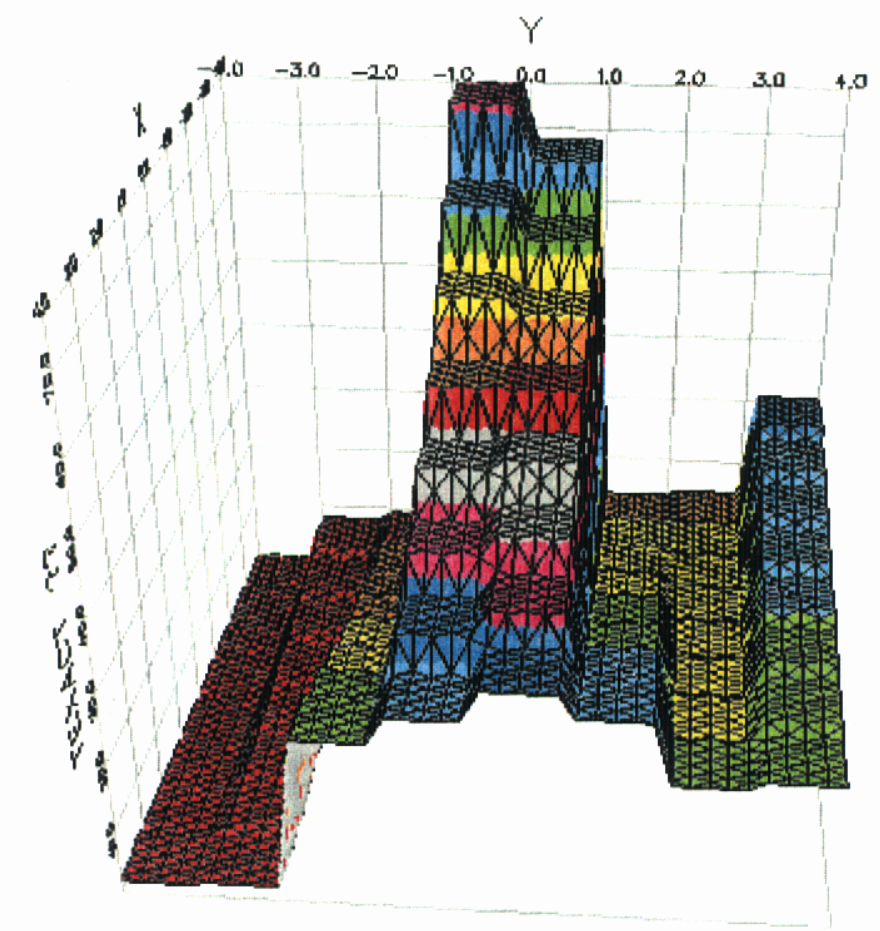

Figure B-3: Plot of synthetic transmissivity used to generate the ground-truth head solution. 


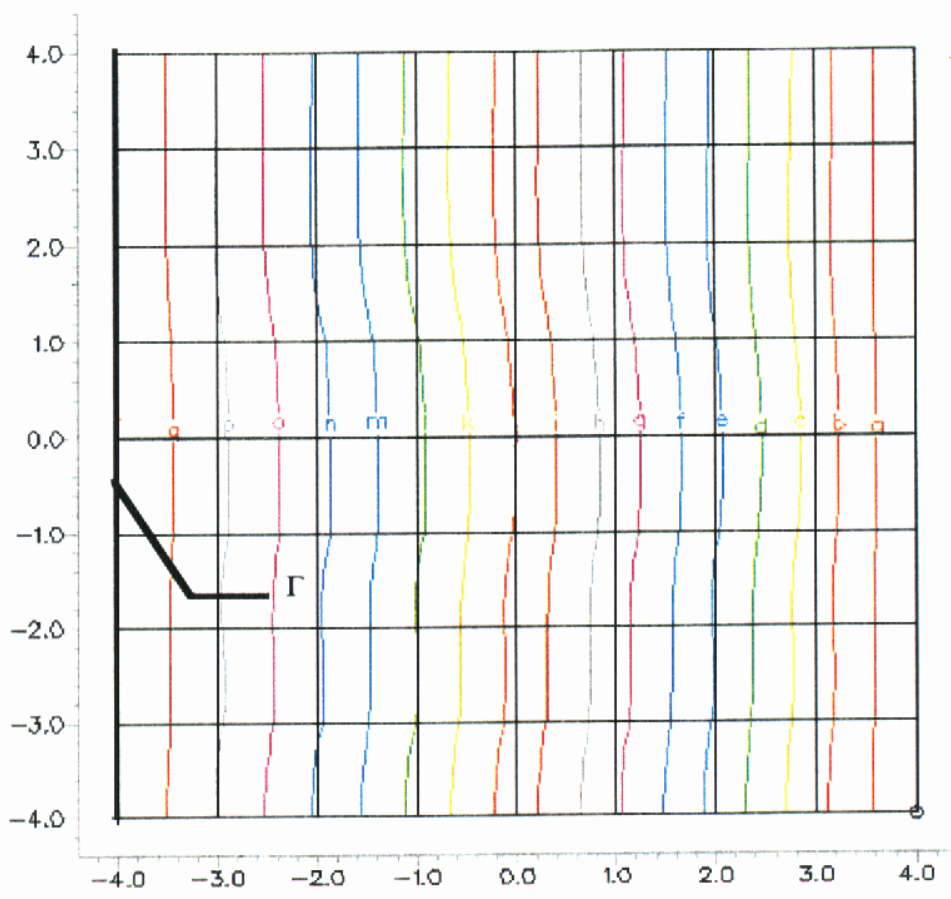

Figure B-4: Isocontour plot of the ground-truth solution $h(x, y)$. The contours $a, b \ldots, q$ correspond to $h=$ $15,20, \ldots, 95$ respectively.

Sixty-four 'measured' $\mathrm{h}$ data are given only at the center co-ordinates of each of the 64 blocks shown in Figure B-4, and T is given along the side $x=-4 ;(-4 \leq y \leq 4)$. Exact transmissivity values were assigned along $\Gamma$ in the calculation of the particular solution $\Phi$ equation (B-17). Using the factorized methods described previously, the discontinuous transmissivity features of the 'true' solution (Figure B-3) are resolved as shown in Figure B-5 for $T_{g}$ and in Figure B-6 for $T_{\text {calc }}$. As expected, sharp discontinuities in the true $T$ distribution of Figure B-3 are smoothed by the undersampling. The general features of the transmissivity distribution have however been reproduced with surprisingly good fidelity from the information contained in such few observation points, in our opinion. The Maximum Relative Error Norm (MREN) $<245 \%$, and the Absolute Relative Error (ARE) $<19 \%$. Maximum errors, as seen in Figure B-7, occur at the T discontinuities, as anticipated. It is here that adaptive grid PDE solvers apparently demonstrate their worth for calculating gradients with the maximum fidelity that is compatible with supporting measured data. 


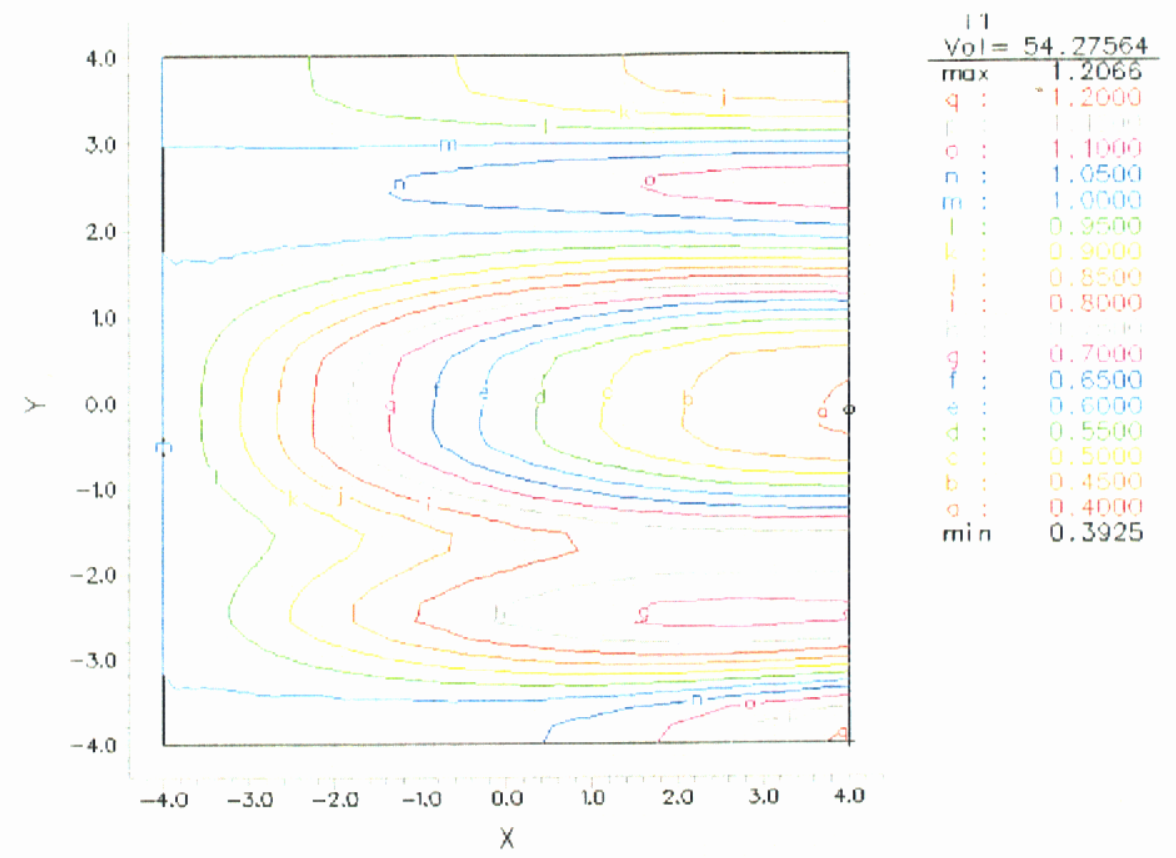

Figure B-5: Isocontour of $T_{g}$ for $T_{g}=1$ along $\Gamma$.

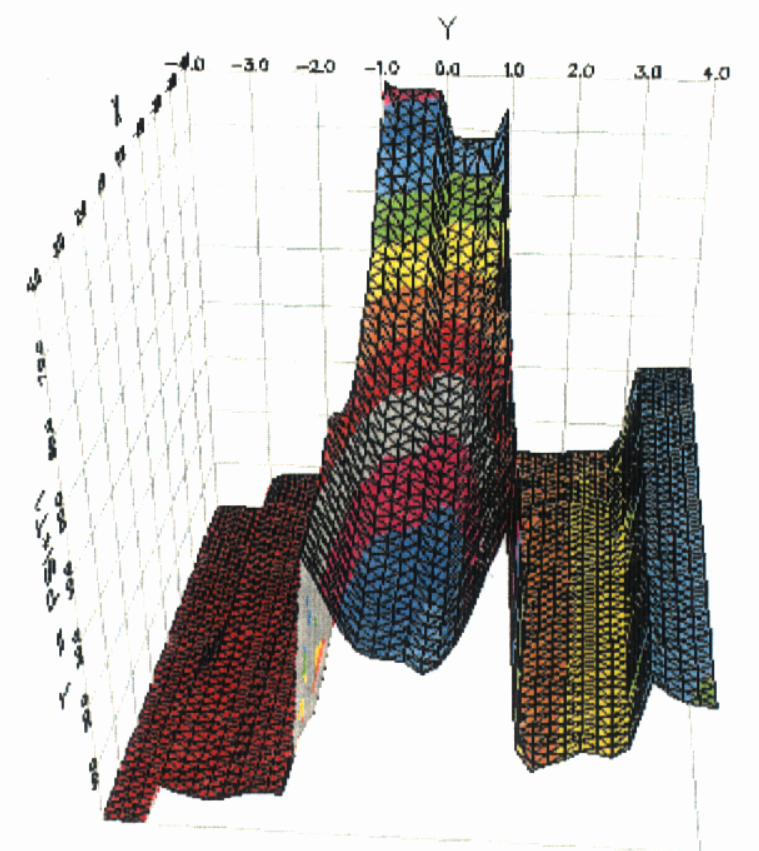

Figure B-6: $T_{\text {calc }}$ surface plot. This estimated transmissivity has the overall shape of the 'true' solution shown in Figure B-3. 


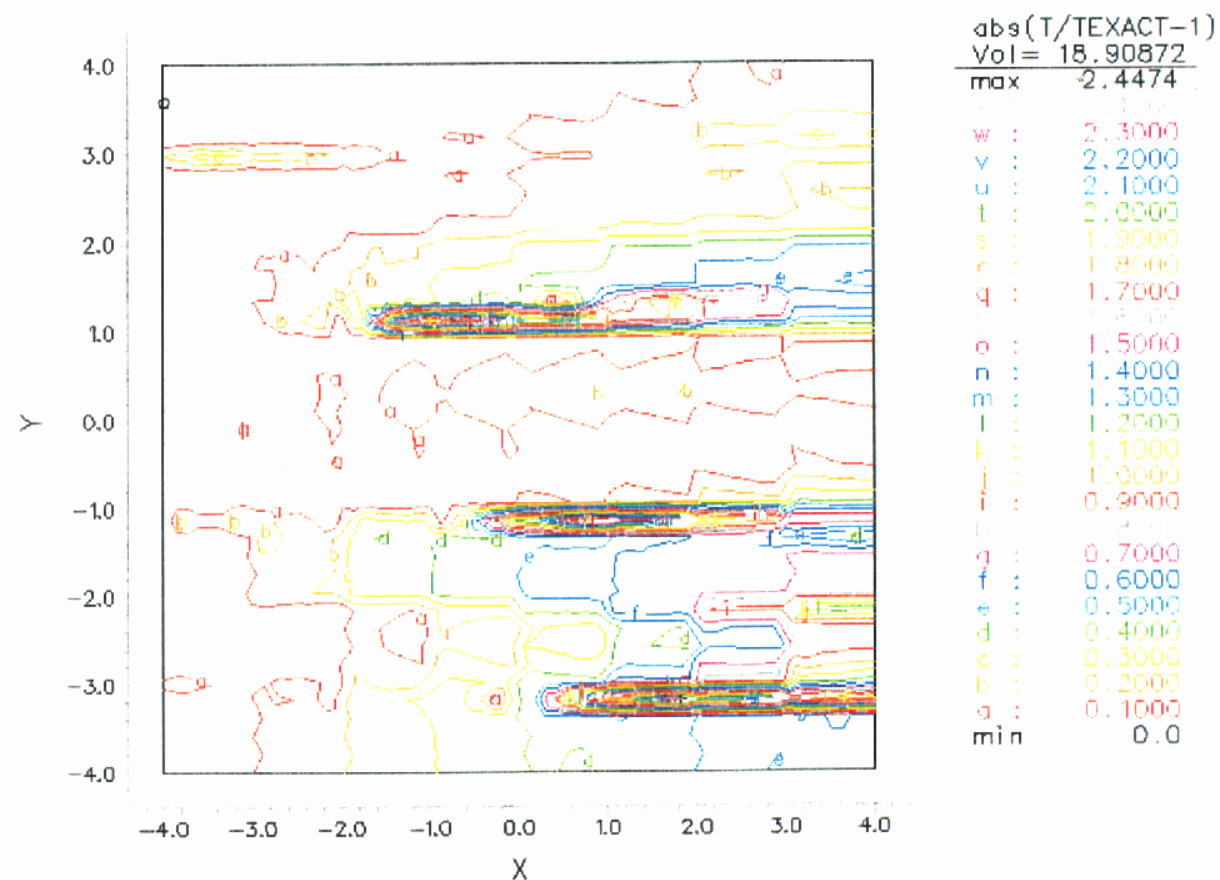

Figure B-7: $\quad$ Plot of MREN for the undersampled $h$ and T. Maximum error occurs along horizontal lines between large changes in $\mathrm{T}$ and does not exceed $245 \%$. The ARE is $<19 \%$. 
This page intentionally left blank 


\section{Appendix C: A Technical Description of FlexPDE Toolkit}

FlexPDE is a software tool for the solution of systems of partial differential equations. It offers an integrated problem solving environment, including a problem description language, a finite element numerical modeling facility, and graphical output of solutions. The interactive run-modify-run environment provides convenient, effective development and debugging of models.

\section{Equation Systems}

FlexPDE can treat boundary value and eigenvalue problems in two or three space dimensions, as well as initial/boundary value problems in two or three space dimensions plus time. Systems may contain equations of different types; e.g., elliptic, parabolic, hyperbolic, algebraic, etc. (Users must be cognizant of appropriate initial values, boundary values, and other mathematical requisites.) The equations are assumed to be of first or second order in space, and first order in time. Equations of higher order must be rewritten as systems of equations of lower order. Equations may be linear or nonlinear, and FlexPDE will automatically apply a solution method which is appropriate to the system. The number of simultaneous equations is limited only by the resources of the computer.

\section{Boundary Conditions}

Boundary conditions may be specified as arbitrary combinations of "value" and "natural" conditions.

"Value" boundary conditions specify the value of a given dependent variable as a function of constants, spatial coordinates, and values or derivatives of dependent variables. "Natural" boundary conditions depend for their meaning on the way the equations are written, but in the usual case refer to the specification of a boundary flux. Natural boundary conditions are given as functions of constants, spatial coordinates, and values or derivatives of dependent variables. Consider for example the heat equation $\operatorname{div}\left(-\mathrm{K}^{*} \operatorname{grad}(\mathrm{T})\right)=\mathrm{H}$. Application of the divergence theorem to the left side reduces it to the surface integral of $\left(-\mathrm{K}^{*} \operatorname{grad}(\mathrm{T})\right)$, which is the meaning of the natural boundary condition, ie. the surface flux.

\section{Problem Domains}

Problem domains can be arbitrarily complex in two space dimensions, but contiguity is assumed. Two-dimensional domains may be made up of an arbitrary number of regions, with differing parameter definitions in all or any region. Three dimensional domains are constructed as layered extrusions of two-dimensional domains, and so are more restricted. Any number of layers may be specified, and material parameters may be different in any layer of any region. I ayer interfaces may be non-planar, specified by arbitrary functions of $2 \mathrm{D}$ spatial coordinates, but must not intersect.

\section{Problem Descriptors}

FlexPDE uses a sophisticated grammar-based input format, which allows problem descriptions to be written in a compact and readable form, following very closeiy the mathematical description of the 
equations and parameters. The problem domain is specified by walking the region boundaries, attaching boundary conditions as appropriate.

\section{Symbolic Equation Processing}

Equations and definitions presented in the problem descriptor are subjected to various symbolic operations internal to FlexPDE. This includes expansion of vector differential operators (div and curl), simplification of arithmetic, and formation of Galerkin integrals and Jacobian matrices. All these derived equations are held internally as computation trees, and are evaluated as needed during the progress of the solution. In this portion of its processing, FlexPDE is similar to a symbolic algebraic processor or a language compiler.

\section{Finite Element Model}

FlexPDE forms a Galerkin finite element model of the equation system, using quadratic or cubic basis functions involving nodal values of system variables only ${ }^{4}$. This model assumes that the dependent variables are continuous over the problem domain, but does not require or impose continuity of derivatives of the dependent variables. Second-order terms in the equations will give rise to various forms of flux continuity (through surface integrals generated by integration by parts), and these conditions will be imposed in an integral scnse over the cell faces.

\section{Numerical Solutions}

During the symbolic processing of the equation system, FlexPDE detects whether the problem is time dependent or steady-state, linear or nonlinear, symmetric or nonsymmetric. Appropriate solution algorithms are then applied to effect an efficient solution. Symmetric steady-state systems attempt to apply an incomplete Choleski conjugate gradient iteration method (ICCG); if this fails, or if the system is time-dependent, a preconditioned conjugate gradient technique is applied. Nonsymmetric systems use a Lanczos (bi-conjugate gradient) iteration method. Nonlinear systems use a NewtonRaphson iteration with backtracking, while linear systems perform an iteration on residuals.

\section{Adaptive Meshes}

FlexPDE automatically generates an unstructured computational mesh of triangles or tetrahedra which fill the domain and match region boundaries. If the solution generated on the initial mesh fails

\footnotetext{
${ }^{4}$ There are ramifications to this model which require care on the part of the user. In electromagnetics, for example, the normal component of $\mathrm{D}$ is continuous across material interfaces, while the tangential component of $E$ is continuous. It is not possible in general to satisfy both of these conditions if the field components themselves are chosen as the system variables. There are two ways to address this difficulty. First is to pose the problem in terms of potentials and not field components. The potential equation $\operatorname{div}\left(\mathrm{eps}^{*} \operatorname{grad}(\mathrm{V})\right)=$ rho accurately represents all the physical requirements of the system. The cell-face integral of the normal component of $\mathrm{D}$ will be continuous across material interfaces (this follows from application of the divergence theorem to the PDE), and the tangential component of $E$ will be everywhere continuous on the interface (since $\mathrm{V}$ is single-valued on the interface). If the user is still determined to model field components instead of potentials, then he must restrict himself to problems in which the continuity requirements can be met, ie., in which some of the field components are missing.
} 
to meet the user-specified error tolerance in every cell, then the mesh is adaptively refined and a new solution is found, until the requested accuracy criterion is met. In time dependent problems, meshes will be refined where necessary, and un-refined where no longer required, so that mesh density will follow moving fronts.

\section{Graphical Output}

Graphical output can be requested for any function of independent and dependent variables and constants. Available graphic formats include contour plots, surface plots, elevations (line-outs), vector fields, and deformed meshes. Arbitrary function values, including area and surface integrals, can be reported on any plot, and a summary page can be written with reports of arbitrary function values.

\section{Availability}

FlexPDE is a product of PDE Solutions Inc. More information can be found on Internet at www.pdesolutions.com. 
This page intentionally left blank 


\section{Appendix D: Listing of Problem Setup Commands}

FlexPDE Descriptor File for the forward Dome Model Problem (Figures 8-14 of text)

\{DMHSS1 --> Heterogeneous Sand (K=5.e-13)/Silt (K=5.e-14) Dome Problem. \}

TITLE 'OW Water-Drive Heterogeneous sand/silt'

\section{SELECT}

errlim $=2 . e-3$

nodelimit $=8000$

upwind $=$ off

firstparts

nrupdate $=4$

tcenter $=1$
! Maximum error bound

! Do not use FlexPDE upwinding

! Force integration by parts of first order terms

! Maximum number of Newton-Raphson Updates

! Use the fully implicit BCE method

VARIABLES

$\mathrm{s}, \mathrm{p}$

! Main system variables: Saturation s and Pressure p.

COORDINATES

cartesian ('x','z')

! Default coordinate system is $(\mathrm{x}, \mathrm{y})$

\section{DEFINITIONS}

p0 $=\quad$ table('dmhom1p.p01') saturation

$\mathrm{s} 0=0$

muo $=4 . e-3$

muw $=1 . e-3$

Kref $=5 \cdot e-13$

$\mathrm{K}=\mathrm{Kref}$

$\mathrm{M}=\mathrm{S}^{\wedge} 2 / \mathrm{muw}+(1-\mathrm{S})^{\wedge} 2 / \mathrm{muo}$

rhow $=1 . \mathrm{e} 3$

rhoo $=.8 \mathrm{e} 3$

$\mathrm{g}=9.8$

$\operatorname{grad} z=\operatorname{vector}(0,1)$

Mbar $=$ rhow $^{*} \mathrm{~S}^{\wedge} 2 / \mathrm{muw}+\mathrm{rhoo}^{*}(1-\mathrm{S})^{\wedge} 2 /$ muo

$\mathrm{f}=\mathrm{S}^{\wedge} 2 / \mathrm{muw} / \mathrm{M}$

$\mathrm{krw}=\mathrm{S}^{\wedge} 2 / \mathrm{muw}$

$\mathrm{V}=-\mathrm{K}^{*}\left(\mathrm{M}^{*} \operatorname{grad}(\mathrm{p})+\mathrm{Mbar} \mathrm{g}^{*} \operatorname{gradz}\right)$

$\mathrm{VW}=-\mathrm{K}^{*} \mathrm{krw}^{*}\left(\operatorname{grad}(\mathrm{p})+\right.$ rhow* $\left.\mathrm{g}^{*} \operatorname{gradz}\right)$ base

Pin $=1.5 \mathrm{e} 6-$-rhow ${ }^{*} \mathrm{~g}^{*} \mathrm{z}$ ! hydrostatic pressure along vertical sides of rectangle

Pout $=\operatorname{Pin} / 2$

kmeter $=100$

hkmeter $=\mathrm{kmeter} / 2$

$\mathrm{w} 20=.2 *$ hkmeter
I Read table of pressure variable for zero

everywhere. Could use instead $\mathrm{p} 0=1\}$

! Initial Saturation

! oil viscosity in pascal second

! water ........

! Sand absolute permeability $\mathrm{m}^{\wedge} 2$

! Total relative permeability

! Water density $\mathrm{Kg} / \mathrm{m}^{\wedge} 3$

! Oil density

! Gravitational acceleration $\mathrm{m} / \mathrm{s}^{\wedge} 2$
! extraction well pressure

! Dome diameter $=$ length of rectangle base

!width of rectangle base 

crown

frac $=h \mathrm{hkmeter} / 8 \quad !$ distance of well center along axis $\mathrm{x}=\mathrm{hkmeter}$ from dome

tfrac $=2 *$ frac

$\mathrm{hcz}=$ ! $\quad$ ! Radius of extraction well

$\mathrm{h} 1=\mathrm{h} \overline{\mathrm{k}}$ meter-frac

phi $=.33$

mag $=-1 / \operatorname{sqrt}\left(\mathrm{dx}(\mathrm{p})^{\wedge} 2+\mathrm{dz}(\mathrm{p})^{\wedge} 2\right)$

$\mathrm{npx}=\mathrm{dx}(\mathrm{p})^{*} \mathrm{mag}$

npy $=\mathrm{dz}(\mathrm{p}) * \mathrm{mag}$

eps $=2 \cdot e-7$

area $=1$

truearea $=$ integral(area)

sint $=$ integral $(\mathrm{s}) /$ ruearea

$x 1=-6 \quad y 1=4 \quad x 2=-3 \quad y 2=-5$

INITIAL VALUES

$$
\begin{aligned}
& \mathrm{s}=\mathrm{s} 0 \\
& \mathrm{p}=\mathrm{p} 0
\end{aligned}
$$

EQUATIONS

$$
\begin{array}{ll}
\text { phi*dt(s) }+\operatorname{div}(V W)-\text { eps }^{*} \operatorname{div}(\operatorname{grad}(\mathrm{s}))=0 & \text { ! Saturation PDE } \\
\operatorname{div}(\mathrm{V})=0 & \text { ! Pressure PDE }
\end{array}
$$

\section{BOUNDARIES}

\section{REGION 1}

boundary

\{ Describe the outline boundary of half circle atop a rectangle base. Assign

$$
\text { area }=1
$$

conditions as you move along

$$
\begin{aligned}
& \text { start }(0,0) \quad \text { natural }(\mathrm{p})=0 \text { natural }(\mathrm{s})=0 \text { line to }(\mathrm{kmeter}, 0) \\
& \text { value }(\mathrm{p})=\operatorname{Pin} \text { value }(\mathrm{s})=1 \quad \text { line to }(\mathrm{kmeter}, \mathrm{w} 20) \\
& \text { natural }(\mathrm{p})=0 \text { natural }(\mathrm{s})=0 \\
& \operatorname{arc}(\mathrm{center}=\mathrm{hkmeter}, \mathrm{w} 20) \text { angle }=180 \\
& \operatorname{value}(\mathrm{p})=\operatorname{Pin} \text { value }(\mathrm{s})=1 \quad \text { finish }
\end{aligned}
$$

\section{REGION $2 \quad \mathrm{~K}=\mathrm{Kref} / 10$}

\{ Describe baffle-like regions. No BCs assignment is required \}

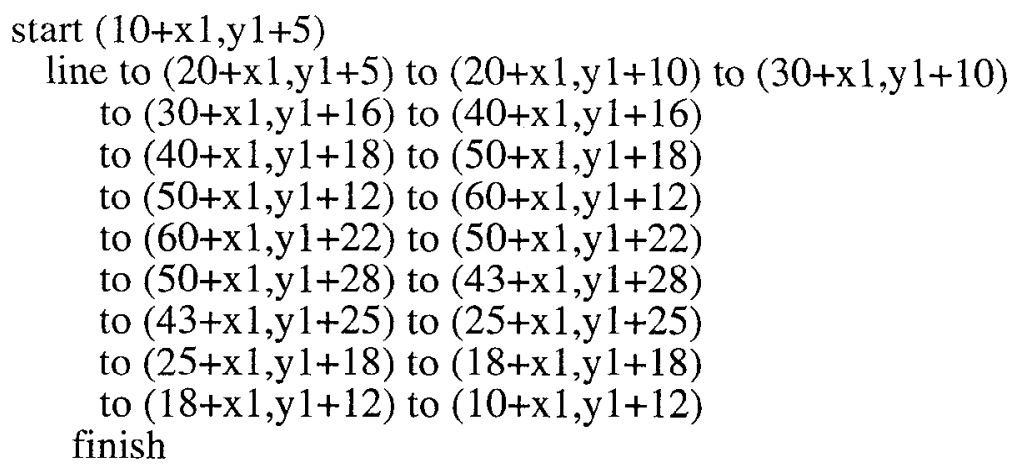


to $(90+\mathrm{x} 2, \mathrm{y} 2+32)$ to $(90+\mathrm{x} 2, \mathrm{y} 2+25)$

to $(91+\mathrm{x} 2, \mathrm{y} 2+25)$ to $(96+\mathrm{x} 2, \mathrm{y} 2+25)$

to $(96+\mathrm{x} 2, \mathrm{y} 2+35)$ to $(84+\mathrm{x} 2, \mathrm{y} 2+35)$

finish

to $(84+\mathrm{x} 2, \mathrm{y} 2+40)$ to $(53+\mathrm{x} 2, \mathrm{y} 2+40)$

EXCLUDE 3 area $=0$

\{ Exclude the well interior region. Assign pressure BCs \} start (hkmeter-hcz,hkmeter+w20-frac) value(p)=Pout $\operatorname{natural}(\mathrm{s})=\operatorname{normal}(\mathrm{VW})$ $\operatorname{arc}($ center $=h k m e t e r, h k m e t e r+w 20-$ frac $)$ angle $=360$ finish

TIME 0 to $86400^{*} 2000$ by $10 \quad$ ! Time interval specification

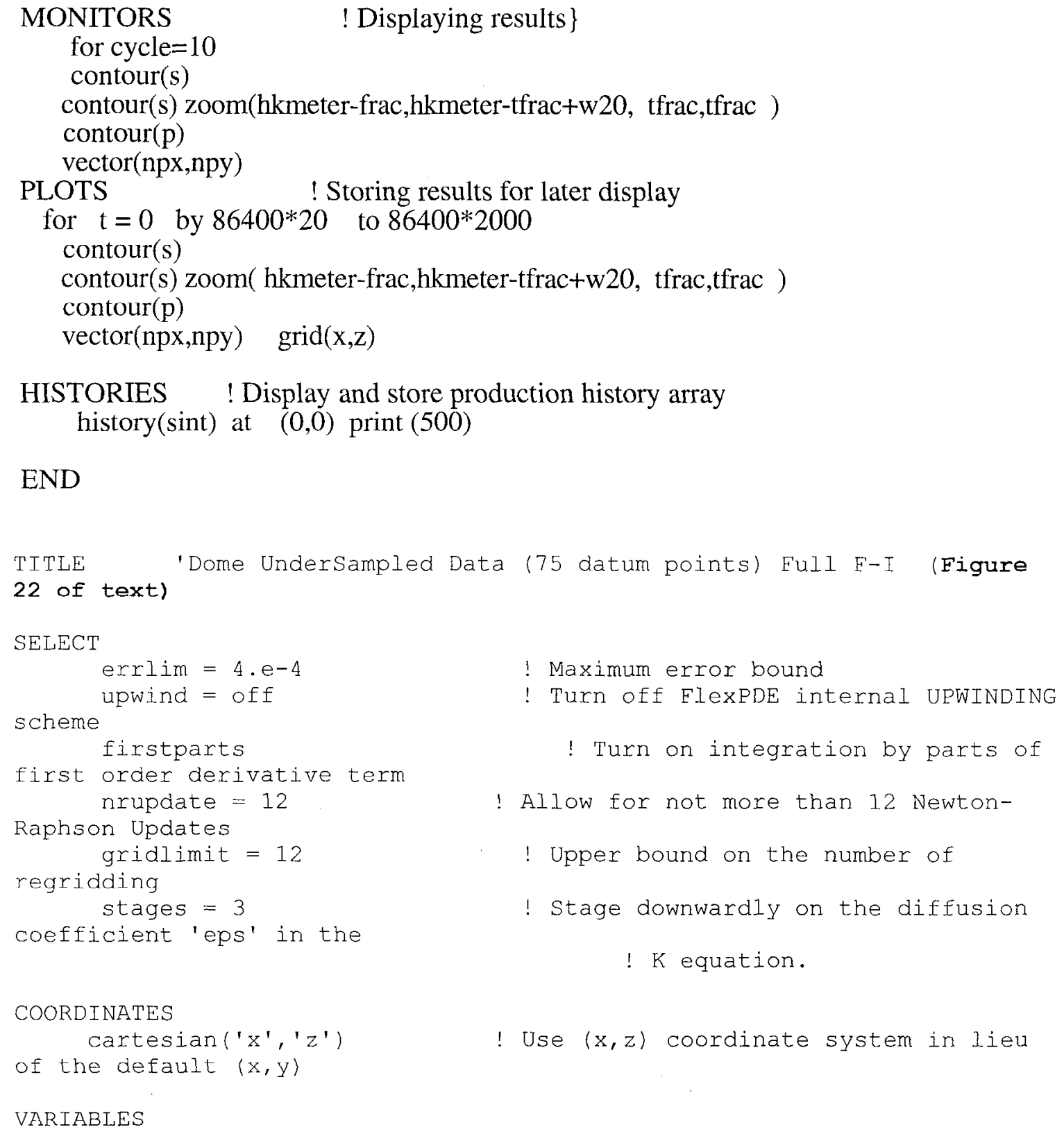




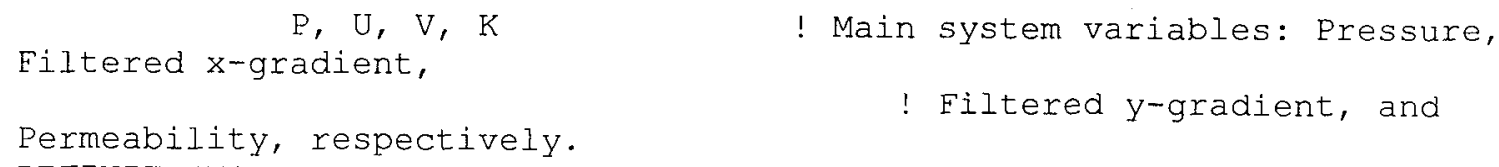


INITIAL VALUES

$\mathrm{P}=\mathrm{p} 0 \quad \mathrm{U}=1 \quad \mathrm{~V}=\mathrm{I} \quad \mathrm{K}=$ Kref $\quad$ I Starting values of $\mathrm{P}-$ $K$ variables. While in linear problems these starting values are fairly arbitrary, a judicious choice is usually required in non linear problems, as is the case EQUATIONS

Pressure PDE $\operatorname{div}($ vee $)=0$ here.

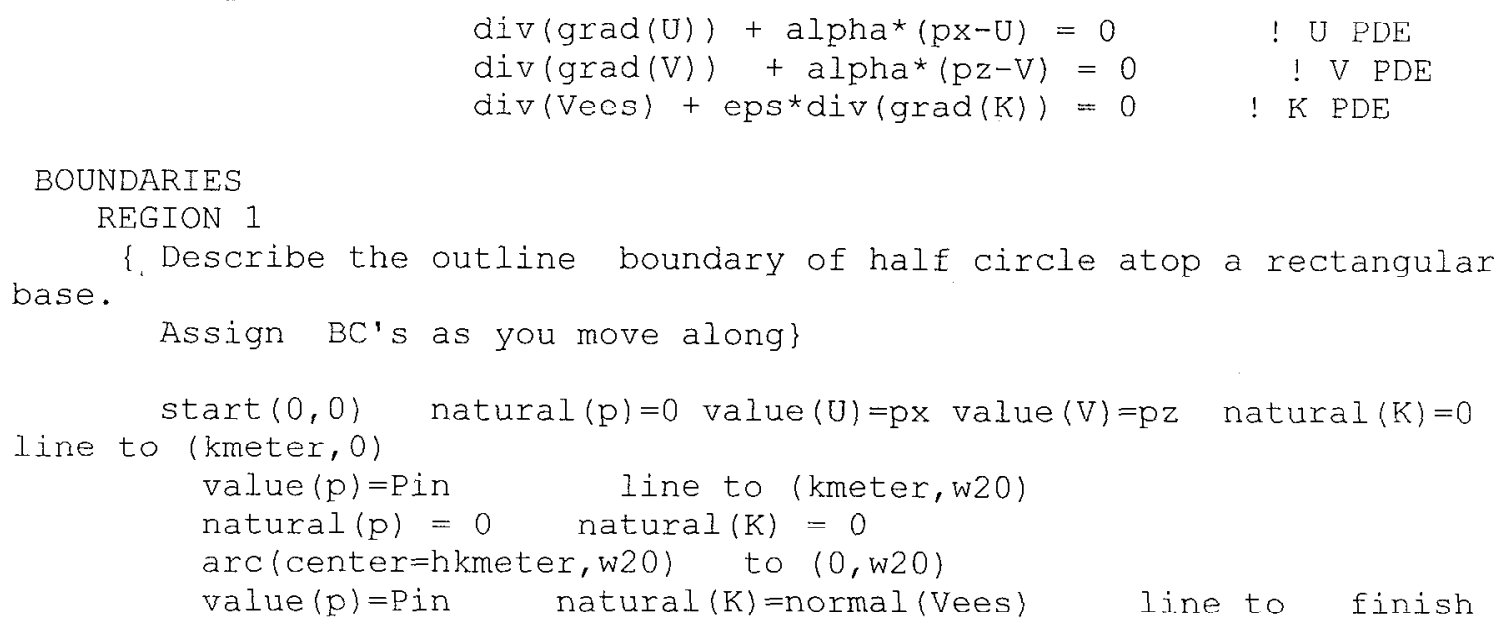

EXCLUDE 2

! Exclude the well internal region from domain and assign BC's

start (hkmeter-4*hcz, hkmeter-fractw20) value $(p)=p 0$ value $(u)=p x$ $\operatorname{value}(\mathrm{V})=\mathrm{pz}$

$\operatorname{value}(K)=\operatorname{Kref}$

$\operatorname{arc}($ center $=$ hkmeter, hkmeter-fractw20) angle=360 finish

\section{$\stackrel{!}{\text { APRIORI }}$}

DEFINE ALI POINTS INSIDE DOMAIN AT WHICH PRESSURE TS KNOWN

! First Row fixed point for pinning of $P$

Fixed Point $(x 0+d e l x, z 0+$ del $z)$ point value $(P)=p 0$

Fixed point $(x 0+2 *$ del $x, z 0+d e l z)$ point value $(P)=p 0$

Fixed Point $\left(x 0+3^{*}\right.$ delx, $z 0+$ del $\left.z\right)$ point value $(P)=p 0$

Fixed Point $\left(x 0+4^{*}\right.$ del $x, z 0+$ del $\left.z\right)$ point value $(P)-p 0$

Fixed Point $(x 0+5 *$ delx, $z 0+$ del $z)$ point value $(P)=p 0$

Fixed Point $(x 0+6 *$ delx, $z 0+\operatorname{del} z)$ point value $(P)=p 0$

Fixed Point $(x 0+7 *$ delx, $z 0+\operatorname{del} z)$ point value $(P)=p 0$

Fixed Point $(x 0+8 *$ del $x, z 0+$ del $z)$ point value $(P)=p 0$

Fixed Point $(x 0+9 *$ delx, $z 0+$ del $z)$ point value $(P)=p 0$

! Second Row fixed point for pinning of $P$

Fixed Point $\left(x 0+\right.$ delx, $z 0+2^{\star}$ del $\left.z\right)$ point value $(P)=p 0$

Fixed Point $(x 0+2 *$ delx, $z 0+2 *$ del $z)$ point value $(P)=p 0$

Fixed Point $(x 0+3 *$ del $x, z 0+2 *$ del $z)$ point value $(P)=p 0$

Fixed Foint $(x 0.4 *$ delx, $z 0+2 *$ del $z)$ point value $(E)=p 0$ 
Fixed Point $(x 0+5 *$ del $x, z 0+2 *$ del $z)$

Fixed Point $(x 0+6 * \operatorname{del} x, z 0+2 * \operatorname{del} z)$

Fixed Point $\left(x 0+7^{*}\right.$ delx, $z 0+2^{\star}$ del $z$ )

Fixed Point $\left(x 0+8 *\right.$ del $x, 20+2^{\star}$ del $\left.z\right)$

Fixed Point $(x 0+9 * d e 1 x, 20+2 *$ del $z)$ point value $(P)=p 0$ point value $(P)=p 0$ point value $(P)=p 0$ point $\operatorname{value}(\mathrm{P})=\mathrm{p} 0$ point value $(P)=p 0$

! Third Row fixed point for pinning of $P$

Fixed Point $\left(x 0+\right.$ delx, $z 0+3^{\star}$ del $\left.z\right)$ point value $(P)=p 0$

Fixed Point $(x 0+2 *$ delx, $z 0+3 *$ del $z)$ point value $(P)=p 0$

Fixed Point $(x 0+3 *$ delx, $z 0+3 *$ del $z)$ point value $(P)=p 0$

Fixed Point $\left(x 0+4^{*}\right.$ del $x, z 0+3^{*}$ del $\left.z\right)$ point value $(P)=p 0$

Fixed Point $\left(x 0+5^{*}\right.$ del $x, z 0+3^{*}$ del $\left.z\right)$ point value $(P)=p 0$

Fixed Point $\left(x 0+6^{*}\right.$ delx, $z 0+3 *$ del $\left.z\right)$ point value $(P)=p 0$

Fixed Point $(x 0+7 *$ delx, $z 0+3 *$ del $z)$ point value $(P)=p 0$

Fixed Point $\left(x 0+8^{*}\right.$ delx, $z 0+3^{*}$ del $\left.z\right)$ point value $(P)=p 0$

Fixed Point $\left(x 0+9^{\star}\right.$ delx, $\left.z 0+3 * d c l z\right)$ point value $(P)=p 0$

! Fourth Row fixed point for pinning of $P$

Fixed Point $\left(x 0+\operatorname{del} x, z 0+4^{*}\right.$ del $\left.z\right)$ point value $(P)=p 0$

Fixed Point $\left(x 0+2^{\star}\right.$ delx, $z 0+4 *$ del $\left.z\right)$ point value $(P)=p 0$

Fixed Point $\left(x 0+3^{*}\right.$ delx, $z 0+4 *$ del $\left.z\right)$ point value $(P)=p 0$

Fixed Point $(x 0+4 *$ del $x, z 0+4 * \operatorname{del} z)$ point value $(P)=p 0$

Fixed Point $(x)+5^{\star}$ delx, $z 0+4^{\star}$ del $\left.z\right)$ point value $(P)=p 0$

Fixed Point $(x 0+6 *$ del $x, z 0+4 * \operatorname{del} z)$ point value $(P)=p 0$

Fixed Point $(x 0+7 *$ del $x, \angle 0+4 * d e l z)$ point value $(P)=$ p0

Fixed Point $(x 0+8 *$ delx, $z 0+4 *$ del $z)$ point value $(P)=p 0$

Fixed Point $(x 0+9 *$ del $x, z 0+1 * \operatorname{del} z)$ point valuc $(P)=p 0$

! Fifth Row fixed point for pinning of $P$

Fixed Point $\left(x 0+\operatorname{del} x, z 0+5^{*}\right.$ del $\left.z\right)$ point value $(P)=p 0$

Fixed Point $\left(x 0+2^{\star}\right.$ delx, $z 0+5^{\star}$ del $\left.z\right)$ point value $(P)=p 0$

Fixed Point $(x 0+3 *$ delx, $z 0+5 * \operatorname{del} z)$ point value $(P)=p 0$

Fixed Point $(x 0+4 *$ delx, $z 0+5 *$ del $z)$ point value $(P)=p 0$

Fixed Point $\left(x 0+b^{*}\right.$ de $\left.\perp x, z 0+b * d e l z\right)$ point value $(P)=p 0$

Fixed Point $(x 0+6 *$ delx, $z 0+5 *$ del $z)$ point value $(P)=p 0$

Fixed Point $(x 0+7 *$ delx, $z 0+5 *$ del $z)$ poinl value $(P)=p 0$

Fixed Point $(x 0+8 *$ delx, $z 0+5 *$ del $z)$ point value $(P)=p 0$

Fixed Point $(x 0+9 *$ delx, $z 0+5 * d e l z)$ point value $(P)=p 0$

! Sixth Row fixed point for pinning of $P$

Fixed Point $\left(x 0+d e l x, z 0+6^{*}\right.$ del $\left.z\right)$ point value $(P)=p 0$

Fixed Point $\left(x 0+2^{*}\right.$ del $x, z 0+6^{\star}$ del $\left.z\right)$ point value $(P)=p 0$

Fixed Point $\left(x 0+3^{*}\right.$ delx, $z 0+6 *$ del $\left.z\right)$ point value $(P)=p 0$

Fixed Point $(x 0+4 *$ del $x, z 0+6 *$ del $z)$ point value $(P)=p 0$

Eixed Point $\left(x 0+b^{*}\right.$ delx, $z 0+6^{*}$ de $\left.L z\right)$ point value $(P)=p 0$

Fixed Point $\left(x 0+6^{*}\right.$ delx, $z 0+6^{*}$ del $\left.z\right)$ point value $(P)=p 0$

Fixed Point $\left(x 0+7^{*}\right.$ del $x, z 0+6 *$ del $\left.z\right)$ point value $(P)-p 0$

Fixed Point $(x 0+8 *$ delx, $z 0+6 *$ delz) point value $(P)=p 0$

Fixed Point $(x 0+9 *$ delx, $z 0+6 * \operatorname{del} z)$ point value $(P)=p 0$

! Seventh Row fixed point for pinning of $P$ ( 2 points fall outside dome )

! Fixed Point $(x 0+$ delx, $z 0+7 *$ del $z)$ point value $(P)=p 0$

Fixed Point $\left(x 0+2^{*}\right.$ del $x, z 0+7 *$ del $\left.z\right)$ point value $(P)=p 0$

Fixed Point $\left(x 0+3^{*} \operatorname{del} x, z 0+7 * \operatorname{del} z\right)$ point value $(P)=p 0$

f'ixed point $(x 0+4 *$ del $x, z 0+7 *$ del $z)$ point value $(P)=p 0$

Fixed Point $(x 0+5 * \operatorname{del} x, z 0+7 * \operatorname{del} z)$ point value $(P)=p 0$

Fixed Point $\left(x 0+6^{*} \operatorname{del} x, z 0+7 * \operatorname{del} z\right)$ point value $(P)=p 0$ 


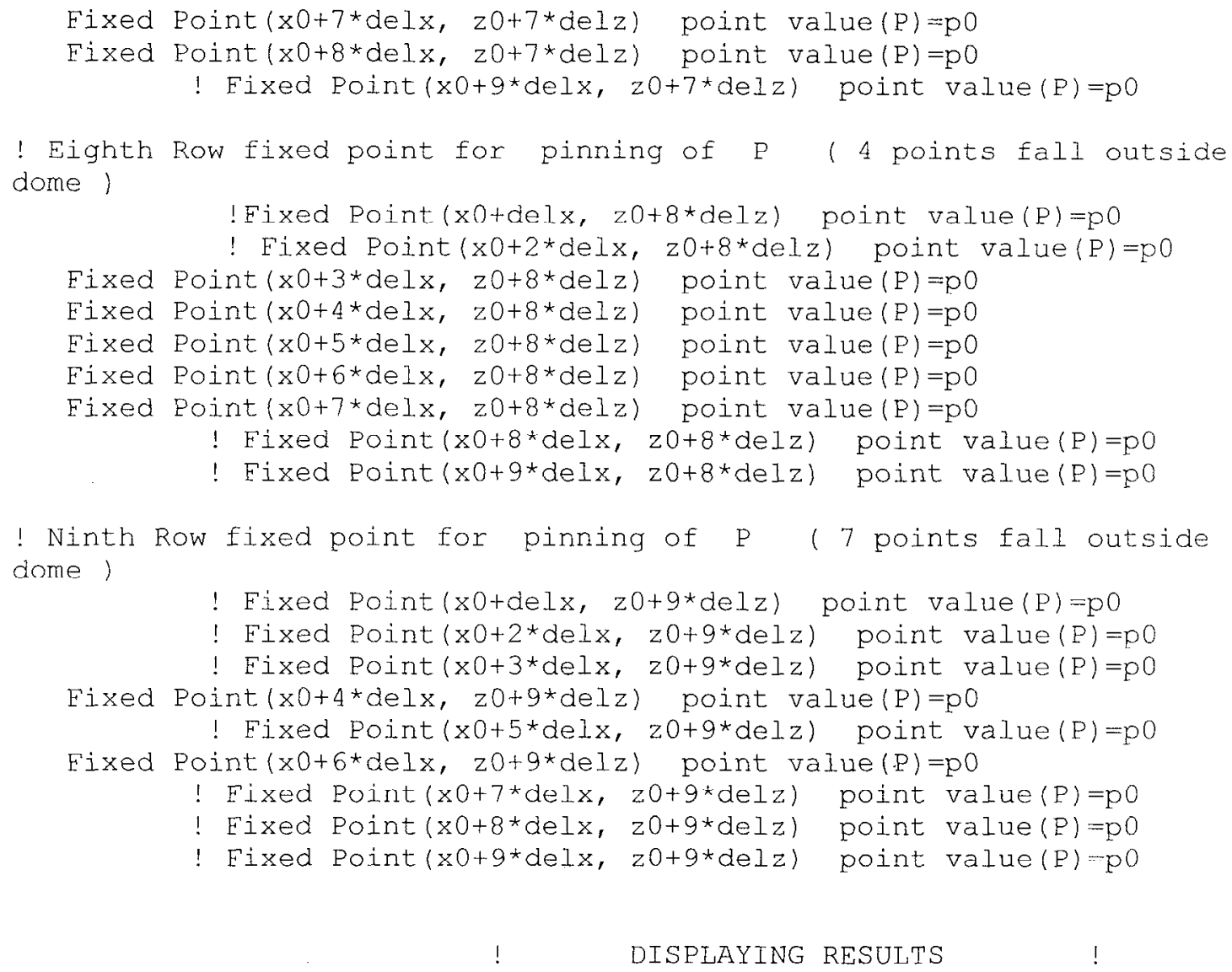

PLOTS

$\operatorname{grid}(\mathrm{x}, \mathrm{z})$

contour $(p)$

contour (K)

contour $(p 0)$

contour (U)

contour $(K)$ painted

contour (V)

contour ( $\mathrm{px}) \quad$ contour $(\mathrm{U}-\mathrm{px})$

contour $(p z) \quad$ contour $(V-p z)$

END

TITLE 'Dome UnderSampled 9 By 9 Full F-I Nov 1098 '

SELECT

$$
\begin{aligned}
& \text { errlim }=4 \cdot e^{-4} \\
& \text { upwind }=\text { off }
\end{aligned}
$$

scheme

firstparts

first order derivative term
! Maximum error bound

! Turn off FlexPDE internal UPWINDING

! Turn on integration by parts of 


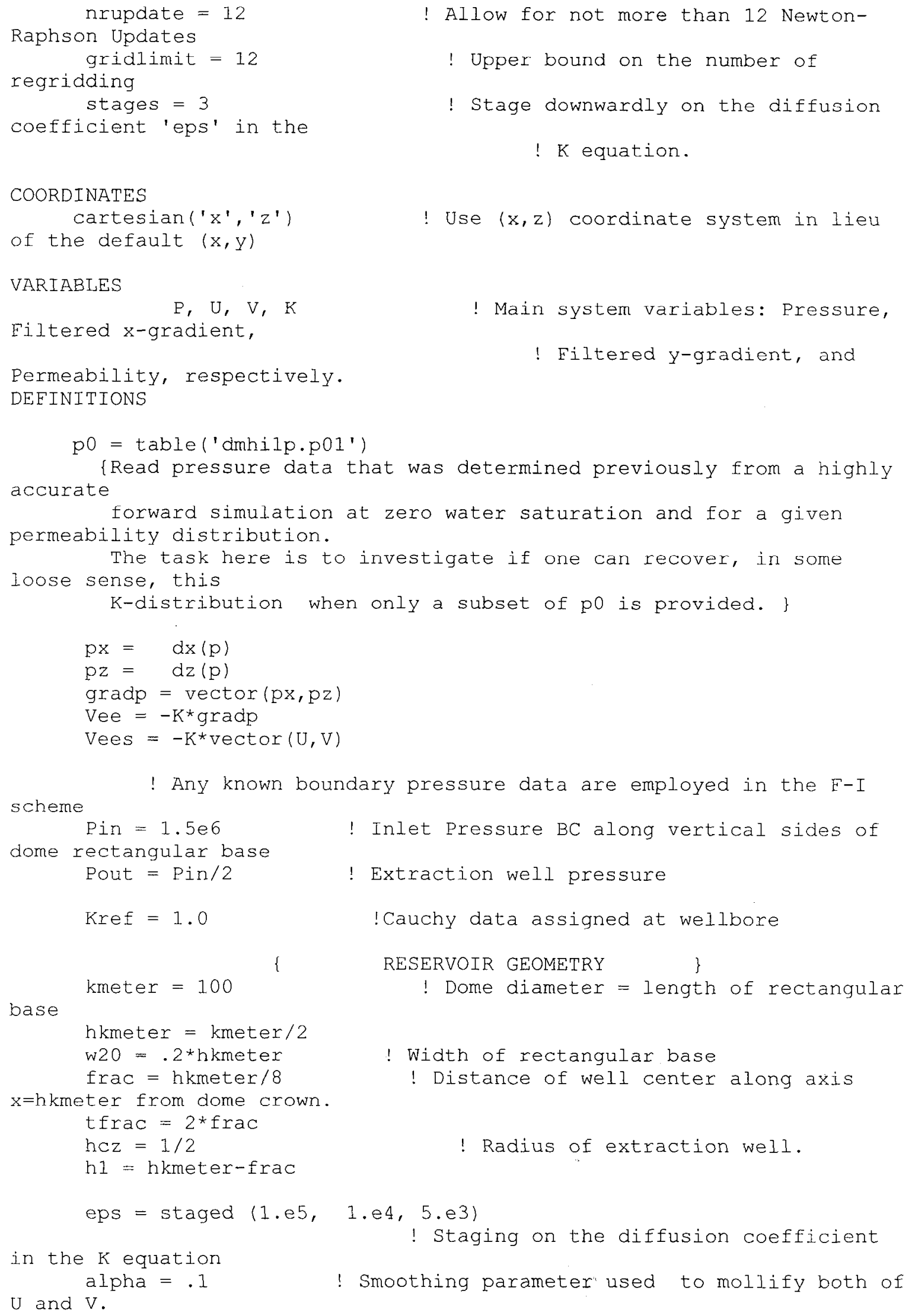


\{ The next four parameters are used in creating a uniform 9 by 9 latticc at

FlexPDF,

which pressure values are assumed known. More recent versions of

are capable of generating these types of lattices along with the assignment of

arbitrary nodal pressure conditions. \}

$\mathrm{xO}=0$

$z 0=0$

delx $=.1 *$ kmeter

delz $=.1 *($ w20+hkmeter $)$

\section{INITIAL VALUES}

$\mathrm{P}=\mathrm{p} 0 \quad \mathrm{U}=1 \quad \mathrm{~V}=1 \quad \mathrm{~K}=$ Kref $\quad$ S Starting values of $\mathrm{P}-$ $K$ variables. While in linear

are fairly arbitrary, a judicious

problems these starting values

non linear problems, as is the case choice is usually required in

\section{EQUATIONS}

Pressure PDE

$$
\operatorname{div}(\mathrm{VCC}) \quad-\quad 0
$$

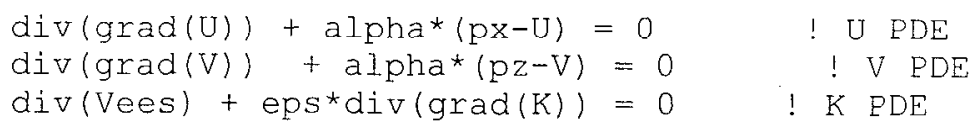

! Exclude the well internal region from domain and assign BC's 
Fixed Foint $\left(x 0+3^{*}\right.$ del $x, z 0+$ del $\left.z\right)$

Fixed Point ( $x 0+4^{*}$ delx, $\left.z 0+d e l z\right)$

Fixed Foint $(x 0+5 *$ del $x, z 0+$ del $z)$

Fixed Point $\left(x 0+6^{*}\right.$ del $x, z 0+$ del $\left.z\right)$

Fixed Point $(x 0+7 *$ del $x, z 0+\operatorname{del} z)$

Fixed Point (x0+8*delx, z0+delz)

Fixed Point $(x 0+9 *$ del $x, z 0+d e l z)$ point $\operatorname{value}(\mathrm{P})=\mathrm{po}$

point value $(P)=p 0$

point value $(P)=p 0$

point value $(P)=p 0$

point value $(P)=\mathrm{p} 0$

point value $(P)=p 0$

point value $(P)=p 0$

! Second Row fixed point for pinning of $P$

Fixed Point $\left(x 0+\operatorname{del} x, z 0+2^{\star}\right.$ del $\left.z\right)$ point value $(P)=p 0$

Fixed Point $(x 0+2 *$ del $x, z 0+2 *$ del $z)$ point value $(P)=p 0$

E'ixed Point $\left(x 0+3^{*}\right.$ delx, $z 0+2 *$ del $\left.z\right)$ point value $(P)=p 0$

Fixed Point $(x 0+4 *$ del $x, 20+2 *$ del $z)$ point value $(P)=p 0$

Fixed Foint $\left(x 0+5^{\star}\right.$ del $x, z 0+2^{\star}$ delz) point value $(P)=p 0$

Fixed Point $\left(x 0+6^{*}\right.$ del $x, z 0+2 *$ del $\left.z\right)$ point value $(P)=p 0$

Fixed Point $(x 0+7 *$ del $x, z 0+2 *$ del $z)$ point value $(P)=p 0$

Fixed Point $(x 0+8 *$ delx, $z 0+2 *$ del $z)$ point value $(P)=p 0$

Fixed Point $(x 0+9 *$ delx, $z 0+2 *$ del $z)$ point value $(P)=p 0$

! Third Row fixed point for pinning of $P$

Fixed Point $(x 0+$ delx, $z 0+3 *$ del $z)$ point value $(P)=p 0$

Fixed Point $(x 0+2 *$ del $x, z 0+3 *$ del $z)$ point value $(P)=p 0$

Eixed Point $\left(x 0+3^{*}\right.$ delx, $z 0+3 *$ del $\left.z\right)$ point value $(\mathrm{P})=\mathrm{p} 0$

Eixed Point $(x 0+4 *$ delx, $z 0+3 * \operatorname{del} z)$ point value $(P)=p 0$

Fixed Point $(x 0+5 *$ delx, $z 0+3 *$ del $z)$ point value $(F)=p 0$

Fixed Point $\left(x 0+6^{*}\right.$ delx, $z 0+3^{*}$ del $\left.z\right)$ point value $(P)=p 0$

Fixed Point $(x 0+7 *$ del $x, z 0+3 *$ del $z)$ point value $(P)=p 0$

Fixed Point $(x 0+8 *$ del $x, 20+3 *$ del $z)$ point value $(P)=p 0$

Fixed Point $\left(x 0+9 *\right.$ del $\left.x, 20+3^{*} \operatorname{del} z\right)$ point value $(P)=p 0$

! Fourth Row fixed point for pinning of $P$

Fixed Point $(x 0+$ delx, $z 0+4 *$ delz) point value $(P)=p 0$

Fixed Point $(x 0+2 *$ delx, $z 0+4 *$ del $z)$ point value $(P)=p 0$

Fixed Point $\left(x 0+3^{*}\right.$ delx, $z 0+4^{*}$ del $\left.z\right)$ point value $(P)=p 0$

Fixed Point $(x 0+4 * \operatorname{del} x, z 0+4 * \operatorname{del} z)$ point value $(P)=p 0$

Fixed Foint $(x 0.15 *$ delx, $z 01.4 *$ delz) point value $(F)=p 0$

Fixed Point $\left(x 0+6^{*}\right.$ delx, $z 0+4 *$ del $\left.z\right)$ point value $(P)=p 0$

Fixed Point $(x 0+7 *$ del $x, z 0+4 *$ de] $z)$ point value $(P)=p 0$

Fixed Point $(x 0+8 *$ del $x, z 0+4 *$ del $z)$ point value $(P)=p 0$

Fixed Point $(x 0+9 *$ delx, $z 0+4 *$ del $z)$ point value $(P)=p 0$

! Fifth Row fixed point for pinning of $\mathrm{P}$

Fixed Point $(x 0+$ del $x, z 0+5 *$ del $z)$ point value $(P)=p 0$

Fixed Point $(x 0+2 *$ del $x, 20+5 *$ del $z)$ point value $(P)=p 0$

Fixed Point $\left(x 0+3^{*}\right.$ del $x, z 0+5^{*}$ del $\left.z\right)$ point value $(P)=p 0$

Fixed Point $\left(x 0+4^{\star}\right.$ del $x, z 0+5^{*}$ del $\left.z\right)$ point value $(P)=p 0$

Fixcd Point $(x 0+5 * d c l x, z 0+5 * d c l z)$ point value $(P)=p 0$

Fixed Point $\left(x 0+6^{\star}\right.$ delx, $z 0+5^{\star}$ delz) point value $(P)=p 0$

Fixed Point $\left(x 0+7^{\star} \operatorname{del} x, z 0+5^{\star} \operatorname{del} z\right)$ point value $(P)=p 0$

Fixed Point $\left(x 0+8^{*} \operatorname{del} x, z 0+5^{*}\right.$ del $\left.z\right)$ point value $(P)=p 0$

Fixed Point $(x 0+9 *$ delx, $z 0+5 *$ del $z)$ point value $(P)=p 0$

! Sixth Row fixed point for pinning of $P$

Fixed Point $\left(x 0+\operatorname{del} x, z 0+6^{*}\right.$ del $\left.z\right)$ point value $(P)=p 0$

Fixed Point $(x 0+2 *$ delx, $z 0+6 *$ del $z)$ point value $(P)=p 0$

Fixed Poinl $(x 0+3 *$ delx, $\angle 0+6 * d e l z)$ point value $(P)=p 0$

Fixed Point $(x 0+4 *$ del $x, z 0+6 *$ del $z)$ point value $(P)=p 0$

Fixed Point $(x 0+5 \star$ delx, $z 0+6 \star d e l z)$ point value $(P)=p 0$ 
ZI-C

aN' 


\section{Appendix E: Black Oil Model for Example Problems}

A black-oil model (BOM) for oil-water systems is formulated here. It is the basis for the two example problems that are considered in the text. One problem is an idealized water-drive scenario on a 2 -D horizontal rectangular domain $(\Omega)$ with zero gravitational forces; and the other is a 2-D vertical oil-dome model with gravitational forces. The flow systems are described by the mean flow equation in all cases, and stochastic residual flux terms are taken to be negligible. Residual fluxes are averaged second-order statistical fluctuation terms that contribute to the rate of change of the mean flow equation. They act like other source terms that may generally appear in the flow equation. Their neglect is not a serious limitation in proofs-of-concept at this stage of development. Additional implications pertaining to this assumption were discussed in Appendix A.

The saturation of phase $l$ is denoted by $\mathrm{S}_{l}, 0 \leq \mathrm{S}_{l} \leq 1$, with

$$
S_{0}+S_{w}=1 \text {. }
$$

The usual convention for the indices, o for oil and w for water, is adopted here. Often, the water saturation is abbreviated as $S$ and is referred to simply as "saturation" without any other qualifications. As in the article by Saad and Zhang [1998], simple parabolic relative permeabilities, $\mathrm{K}_{\mathrm{Tw}}$ and $\mathrm{K}_{\mathrm{ro}}$, are assumed and expressed as

$$
\begin{aligned}
& \mathrm{K}_{\mathrm{rw}}=\mathrm{S}_{\mathrm{w}_{2}}^{2} \\
& \mathrm{~K}_{\mathrm{ro}}=\mathrm{S}_{\mathrm{o}}
\end{aligned}
$$

The mobility, $\lambda_{l}$, of phase $l$ is defined by,

$$
\lambda_{l}=\mathrm{K}_{\mathrm{sat}} \cdot \mathrm{K}_{\mathrm{rl}} / \mu_{l}
$$

where $\mathrm{K}_{\mathrm{sat}}$ is the absolute rock permeability, and $\mu_{l}$ is the viscosity of phase $l$. For ease of writing, the subscript in $\mathrm{K}_{\text {sat }}$ will henceforth be dropped. Thus, in the remaining discussion, $\mathrm{K}$ will denote the saturation-independent permeability coefficient. Because capillary pressure is assumed to be zero, the oil and water pressures are equal and will henceforth be denoted by $\mathrm{P}$. The total mobility, $\mathrm{M}$, is defined as the sum of all mobilities divided by $\mathrm{K}$. It follows from equations $(E-1)-(E-3)$ that $M$ is given by,

$$
\mathrm{M}=\mathrm{S}^{2} / \mu_{w}+(1-\mathrm{S})^{2} / \mu_{\mathrm{o}}
$$

The total velocity $\mathrm{V}$ and the fractional flux $\mathrm{f}$ are defined as,

$$
\begin{gathered}
V=-K \cdot M \operatorname{grad}(P), \\
f=\left[S^{2} / \mu_{w}\right] / M .
\end{gathered}
$$

If the porosity $\varphi$ is taken to be constant and gravity effects are negligible everywhere in $\Omega$, it can be shown that the two PDEs governing a general two-phase flow reduce to,

$$
\begin{gathered}
\varphi \partial_{t} S+\operatorname{div}(V \cdot f)=0, \\
\operatorname{div}(V)=0 .
\end{gathered}
$$


Throughout this discussion, equations $(\mathrm{E}-7)$ and $(\mathrm{E}-8)$ are referred to as the saturation and pressure equations, respectively. This makes sense, since the former describes the saturation evolution for a known pressure distribution and a given initial saturation. And vise versa, the latter determines the pressure $\mathrm{P}$ everywhere for a known saturation distribution and given pressure boundary conditions (BCs). In fact, if the flow is assumed to be one-dimensional, say along the $\mathrm{x}$-axis, then from equation (E-8), $\mathrm{V}$ must be a constant; and equation (E-7) yields the well-known Buckley-Leverett equation in the water saturation, namely,

$$
\varphi \partial_{t} S+V \partial_{x} f=0
$$

It is important to note here the vast distinction in the types of the two PDE's, (E-7) and $(\mathrm{E}-8)$. On the one hand, the pressure equation $(\mathrm{E}-8)$ is a well-behaved elliptic equation. It is, in fact, 'strongly elliptic', since the coefficient $\mathrm{M}(\mathrm{S})$ is bounded from below by the positive constant $1 /\left(\mu_{\mathrm{o}}+\mu_{\mathrm{w}}\right)$. It is thus posed as a boundary value problem (BVP). The saturation equation (E-7), on the other hand, is of the hyperbolic type and should thus be treated as a Cauchy-initial-value problem. That is, the saturation equation is well-posed only if the initial saturation is given everywhere in $\Omega$ along with the saturation history over some 'admissible curve', $\Gamma$. The significance of these distinctions will be discussed more extensively in later technical articles. 


\section{References}

Akcasu, A.Z., and R.K. Osborn (1966), "Application of Langevin's Technique to Space and Energy Dependent Noise Analysis," Nucl. Sci. Eng., 26, pp. 14-25.

Aziz, K. and A. Settari (1983), Petroleum Reservoir Simulation, Elsevier Applied Science (New York)

Backstrom, G. (1994), Fields of Physics on the PC by Finite Element Analysis, Student Litteratur (ISBN 91-44-48671-5), Lund, Sweden or Chartwell Bratt Ltd., (ISBN 086238-382-X) British Library Catalog.

Beran, M. J. (1968), Statistical Continuum Theories, Interscience, New York, NY

Backstrom, G. (1998), Fields of Physics by the Finite Element Method-An Introduction, Student Litteratur ab (ISBN 91-44-00655-1) Box 141, SE22100, Lund, Sweden.

Bracewell, R.N. (1986), The Fourier Transform and Its Applications, Second Ed., Revised, McGraw-Hill, New York, NY.

Carle, S.F. (1996a), A Transition Probability-Based Approach to Geostatistical Characterization of Hydrostratigraphic Architecture, Ph.D. Dissertation, Hydrology Program, Department of Land, Air, and Water Resources, University of California, Davis, CA.

Carle, S.F., and G.E. Fogg (1996b), "Transition Probability-Based Indicator Geostatistics", Math. Geology, 28(4), pp. 453-477.

Carrera, J., and S.P. Neuman (1986a), "Estimation of Aquifer Parameters Under Transient and Steady State Conditions, 1, Maximum Likelihood Method Incorporating Prior Information," Water Resour. Res., 22(2), pp. 199-210.

Carrera, J., and S.P. Neuman (1986b), "Estimation of Aquifer Parameters Under Transient and Steady State Conditions, 2, Uniqueness, Stability, and Solution Algorithms," Water Resour. Res., 22(2), pp. 211-227.

Courant D., and D. Hilbert (1953), Methods of Mathematical Physics, Volumes 1 and 2, Interscience, New York, NY.

Dagan, G. (1989), Flow and Transport in Porous Formations, Springer-Verlag, New York, NY.

Emsellem, Y., and G. deMarsily (1971), "An Automatic Solution for the Inverse Problem," Water Resour. Res., 7(5), pp. 1264-1283.

Frind, E.O., and G.F. Pinder (1973), "Galerkin Solution of the Inverse Problem for Aquifer Transmissivity," Water Resour. Res., 9(5), pp. 1397-1409.

Gelhar, L.W. (1993), Stoschastic Subsurface Hydrology, Prentice-Hall, Englewood Cliffs, NJ.

Gelinas, R. J., S.K. Doss, J.P. Ziagos, and R.G. Nelson (1998), "Enhancing Environmental Restoration Predictive Modeling in Undersampled Environments", LLNL Report UCRL-ID-129568, Lawrence Livermore National Laboratory, Livermore, Calif. 
Gelinas, R.J. (1976), "Comments on the Foundations of Fluctuations in Reactive Systems," Combustion Sci. and Technol., 13, pp. 269-275.

Gelinas, R.J., and R.K. Osborn (1966), "Reactor Noise Analysis by Photon Observation," Nucl. Sci. Eng., 24, pp. 184-192.

Gelinas, R.J., and R.K. Osborn (1967), "Kinetic Theory for the Interpretation of Measurements on Fluctuations in Radiation Distributions in Finite, Inhomogeneous Systems," Phys. Rev., 163, No. 1, pp. 162-174.

Hadamard, J. (1952), Lectures on Cauchy's Problem in Linear Partial Differential Equations, Dover, Mineola, NY.

Hantush, M.S., and C.E. Jacob (1955), "Non-Steady Radial Flow in an Infinite Leaky Aquifer," Am. Geophys. Union Trans., 26(1), pp. 95-100.

Hantush, M.S. (1960), "Modification of the Theory of Leaky Aquifers," The J. of Geophys. Res., 65, pp. 3173-3725.

Harter, T., A. L. Gutjahr, and T.-C. Jim Yeh (1996), "Linearized cosimulation of hydraulic conductivity, pressure head, and flux in saturated and unsaturated, heterogeneous porous media”, Journal of Hydrology, 183,pp. 169-190

Hsu, K.-C., D. Zhang, and S. P. Neuman (1996), "Higher-order correction of effective permeability of heterogeneous porous media, Water Resour. Res., 32(3), pp.571-582

Jea, K.C., and D.M. Young (1983), "On the Simplification of Generalized Conjugate Gradient Methods for Nonsymmetrizable Linear Systems," Linear Algebra and It's Applications, 52/53, pp. 399-417.

John, F. (1982), Partial Differential Equations, Springer-Verlag, New York, NY.

Lanczos, C. (1997), Linear Differntial Operators, Dover Publications, Inc. Mineola, N.Y.

Langevin, P. (1908), "Sur la theorie du movenebt Brownien", Comtes. Rendus Acad. Sci.

(Paris), 146, pp. 530-533

Lax, M., (1966), Discussion of Langevin's technique (title unknown), Rev. Mod. Phys., 38, p. 541.

McGillem, C.D., and G.R. Cooper (1984), Continuous and Discrete Signal and System Analysis, Second Ed., Holt, Rinehart, and Winston, New York, NY.

McLaughlin, D., and L.R. Townley (1996), “A Reassessment of the Groundwater Inverse Problem," Water Resour. Res., 32(5), pp. 1131-1161.

Nelson, R.W. (1961), "In Place Measurement of Permeability in Heterogeneous Media, 2, Experimental and Computational Considerations," J. Geophys. Res., 66(8), pp. 24692478.

Nelson, R.W. (1962), "Conditions for Determining Areal Permeability Distributions by Calculation," J. Soc. Petrol. Eng., 2(3), pp. 223-224.

Nelson, R.G. (1998), See Web Page: www.pdesolutions.com., Sunol, CA. 
Neuman, S. P. and S. Orr (1993), "Prediction of Steady State Flow in Nonuniform Geologic Media by Conditional Moments: Exact Nonlocal Formalism, Effective Conductivities, and Weak Approximation", Water Resources Research, 29(2), pp. 341-364.

Oden, J.T., T. Strouboulis, and P. Devloo (1986), "Adaptive Finite Element Methods for the Analysis of Inviscid Compressible Flow: Part I. Fast Refinement/Unrefinment and Moving Mesh Methods for Unstructured Meshes", Computer Methods in Appplied Mechanics and Engineering, 59, pp. 327-362

Osborn, R.K. (1963), "Kinetic Equations for Fully Ionized, Inhomogeneous Plasmas," Phys. Rev. 130(6), pp. 2142-2155.

Osborn, R.K., and A.Z. Akcasu (1967), "Intensity Fluctuations of the Radiation from a Dispersive Blackbody,” J. Appl. Physics, 38(13), pp. 5165-5172.

Osborn, R.K. (1968), "Stochastic Approach to the Theory of Fluctuations in Plasmas," The Physics of Fluids, 11(3), pp. 595-600.

Protter, M.H., and H.F. Weinberger (1984), Maximum Principles in Differential Equations, Springer-Verlag, New York, NY.

Randall, J. L. (1990), Numerical Methods for Conservation Laws. Lectures in mathematics, Birkhauser

Saad, M. and H. Zhang (1998), "Adaptive Mesh for Two-Phase Flow in Porous Media", Recent Advances in Problems of Flow and Transport In Porous Media, pp. 179 - 192, Edited by J. M. Crolet and M. El Hatri, Kluwer Academic Publishers, (Boston)

Sun, N.-Z. (1994), Inverse Problems in Groundwater Modeling, Kluwer Acad., Norwell, MA.

Van Leer, B. (1977), "Towards the ultimate conservative scheme III, upstream-centered finite difference schemes for ideal compressible flow", Journal of Computational Physics, 23, pp. 263-275

Van Leer, B. (1977), "Towards the ultimate conservative scheme IV, a new approach to the numerical convection”, Journal of Computational Physics, 23, pp. 276-299

Vandenberg, P.M. (1988),"Iterative schemes based on minimization of a uniform error criterion," IEEE Transactions on Antennas and Propagation, AP-36, pp. 1418-1423.

Yeh, W. W-G. (1986), "Review of Parameter Identification Procedures in Groundwater Hydrology: The Inverse Problem," Water Resour. Res., 22, pp. 95-108.

Zimmerman, D. A., G. de Marsily, C. A. Gotway, M. G. Marietta, C. L. Axness, R. L. Beauheim, R. L. Bras, J. Carrera, G. Dagan, P. B. Davies, D. P. Gallegos, A. Galli, J. Gomez-Hernandez, P. Grindrod, A. L. Gutjahr, P. K. Kitanidis, A. M. Lavenue, D. McLaughlin, S. P. Newman, B. S. RamaRao, C. ravenne, and Y. Rubin (1998), "A comparison of seven geostatistically based inverse approaches to estimate transmissivities for modeling advective transport by groundwater flow", Water Resour. Res., 34(6), pp. 1373-1413. 
This page intentionally left blank 